Supporting Information for

\title{
Proteomic Characterization of the Dynamics of Ischemic Stroke in Mice
}

Rong-Fang Gu${ }^{1}$, Terry Fang ${ }^{2}$, Ashley Nelson ${ }^{2}$, Stefka Gyoneva ${ }^{2}$, Benbo Gao ${ }^{1}$, Joe Hedde ${ }^{2 \dagger}$, Kate Henry $^{2}$, Emily Peterson ${ }^{3}$, Linda C. Burkly ${ }^{2 *}$, Ru Wei ${ }^{1^{*}}$

${ }^{1}$ Chemical Biology and Proteomics, Biogen, 225 Binney Street, Cambridge, MA 02142, USA

${ }^{2}$ Genetic and Neurodevelopmental Disorders Research, Biogen, 225 Binney Street, Cambridge, MA 02142, USA

${ }^{3}$ Medicinal Chemistry, Biogen, 225 Binney Street, Cambridge, MA 02142, USA

* Corresponding Authors: Ru Wei, Email: ru.wei@biogen.com, orcid.org/ 0000-0002-7646-

9046; Linda C Burkly, Email: linda.burkly@biogen.com.

$\dagger \mathrm{JH}$ current address: Triplet Therapeutics, One Kendal Square, 1400W, Suite 14201, Cambridge, MA 02139.

\section{List of contents}

Supplementary file 1 (this pdf file):

I. Supplementary methods, including Table S1. TMT 10-plex Design.

II. Supplementary Figures:

a. Figure S1. Differentiated Proteomes in Stroke and Control Samples.

b. Figure S2. Temporal Profiles of 309 Top-Hits in Stroke and Control Samples.

c. Figure S3. Western Blots of Albumin in the Brain Cortex Tissue Samples.

\section{Supplementary file 2 (a separate Excel file):}

Table S2. 3625 Proteins Significantly Changed by Stroke

Table S3. Significantly Enriched Pathways Underlying Stroke-Changed Proteome

Table S4. 309 Top-Hits and Their Correlation to Cytokines and Albumin

Table S5. Correlation among 8 Cytokines and Albumin 


\section{Supplementary methods, including Table S1. TMT 10-plex Design}

\section{DH-MCAO Surgery}

DH-MCAO surgery was performed as following and mice were maintained under anesthesia using $1.5-2 \%$ isoflurane during the entire time of the surgical procedures. In brief, the skin between the orbit and auditory canal was cut and the temporalis muscle was retracted, exposing the skull above the MCA. A small craniotomy was drilled above the MCA and the meninges surrounding the MCA were removed. A low-temperature Bovie cauterizer was used to cauterize the MCA below the bifurcation to occlude all blood flow distal to the cauterization site. The temporalis muscle was placed back over the craniotomy and the incision was sealed using surgical adhesive. Mice were then removed from anesthesia and placed into a hypoxic chamber (Coy Laboratory Products) containing 8\% oxygen and 92\% nitrogen for $1 \mathrm{~h}$. At the end of the hour in hypoxia, mice were returned to their home cage. Sham mice received the same surgical procedure as above, but without cauterization of the MCA. Sham mice were also exposed to $1 \mathrm{~h}$ hypoxia post-surgery.

\section{Albumin Analysis}

For albumin Western blot analysis, brain samples were prepared and normalized as noted above. Samples were mixed with 10x reducing agent (Invitrogen, NP0009) and 4x LDS buffer (Invitrogen, NP0007) to a $1 \mathrm{x}$ final concentration, followed by denaturation at $70{ }^{\circ} \mathrm{C}$ for $10 \mathrm{~min}$ and subsequent separation on 4-12\% Bis-Tris polyacrylamide gel (Invitrogen, WG130BX10) at 150V for $45 \mathrm{~min}$ in 1x MOPS running buffer (Invitrogen, NP0001). Gels were transferred to nitrocellulose membranes (Invitrogen, IB23001), blocked (LI-COR, 927-40000, 2-3 h, at RT), and incubated overnight with a rabbit anti-albumin antibody (Abcam, ab207327; 1:2000) or antiGAPDH antibody (Abcam, ab9484; 1:10,000) at $4{ }^{\circ} \mathrm{C}$ in blocking buffer. Blots were washed $3 \mathrm{x}$ with PBS- $0.1 \%$ Tween 20 for $15 \mathrm{~min}$ and next incubated with secondary antibodies (anti-rabbit IRDye $800 \mathrm{CW}$, anti-mouse IRDye 680RD) at 1:20,000 in blocking buffer for $1 \mathrm{~h}$ at RT. Blots were sequentially washed with PBST and 1x with PBS and imaged on the LI-COR Odyssey. 


\section{Tandem Mass Tag (TMT) 10-plex Labelling and Peptide Fractionation}

For TMT labeling, $20 \mu \mathrm{g}$ of dry peptide digest were chemically labeled with TMT reagents (Thermo Scientific). Peptides were resuspended in $20 \mu \mathrm{L}$ triethylammonium bicarbonate. TMT reagents $(0.8 \mathrm{mg}$ ) were dissolved in $41 \mu \mathrm{L}$ anhydrous $\mathrm{ACN}$, of which $12 \mu \mathrm{L}$ was added to the peptides. Following incubation at room temperature for $1 \mathrm{~h}$, the reaction was quenched using $5 \%$ hydroxylamine for $15 \mathrm{~min}$ at RT. The TMT-labeled samples were pooled at equal peptide ratios into seven TMT-10 sets, followed by vacuum centrifugation to near dryness. To obtain relatively homogenous proteomes among seven TMT-10 sets, we assumed that day- 1 and day-3 samples have relatively similar proteomes, as do day- 7 and day-14, and thus pooled day- 1 and day- 7 samples in three sets, day-3 and day-14 in three sets, and day-28 in one set (Table S1).

Table S1. TMT Proteomics Design

TMT-10_1 TMT-10_2 $\quad$ TMT-10_3 $\quad$ TMT-10_4 $\quad$ TMT-10_5 $\quad$ TMT-10_6 $\quad$ TMT-10_7

\begin{tabular}{|c|c|c|c|c|c|c|}
\hline $\begin{array}{c}\text { strk_Ip, day- } \\
1, n=2\end{array}$ & $\begin{array}{c}\text { strk_lp, day- } \\
1, \mathrm{n}=2\end{array}$ & $\begin{array}{c}\text { strk_Ip, day- } \\
\quad 1, \mathrm{n}=2\end{array}$ & $\begin{array}{c}\text { strk_Ip, day- } \\
3, \mathrm{n}=2\end{array}$ & $\begin{array}{c}\text { strk_Ip, day- } \\
3, n=2\end{array}$ & $\begin{array}{c}\text { strk_lp, day- } \\
3, n=2\end{array}$ & \multirow{2}{*}{$\begin{array}{c}\text { strk_Ip, day- } \\
28, \mathrm{n}=4\end{array}$} \\
\hline $\begin{array}{c}\text { strk_Ct, day- } \\
1, n=1\end{array}$ & $\begin{array}{c}\text { strk_Ct, day- } \\
1, n=1\end{array}$ & $\begin{array}{c}\text { strk_Ct, day- } \\
1, n=1\end{array}$ & $\begin{array}{c}\text { strk_Ct, day- } \\
3, n=1\end{array}$ & $\begin{array}{c}\text { strk_Ct, day- } \\
3, \mathrm{n}=1\end{array}$ & $\begin{array}{r}\text { strk_Ct } \\
3, \mathrm{n}\end{array}$ & \\
\hline $\begin{array}{c}\text { sham_Ip, } \\
\text { day-1, } n=2\end{array}$ & $\begin{array}{c}\text { sham_Ip, } \\
\text { day-1, n=2 }\end{array}$ & $\begin{array}{l}\text { sham_Ip, } \\
\text { day-1, } n=2\end{array}$ & $\begin{array}{l}\text { sham } \\
\text { day-3, }\end{array}$ & $\begin{array}{c}\text { sham_Ip, } \\
\text { day-3, n=2 }\end{array}$ & $\begin{array}{l}\text { sham } \\
\text { day-3, }\end{array}$ & \multirow{2}{*}{$\begin{array}{c}\text { strk_Ct, day- } \\
28, n=4\end{array}$} \\
\hline $\begin{array}{c}\text { strk_Ip, day- } \\
7, \mathrm{n}=2\end{array}$ & $\begin{array}{c}\text { strk_Ip, day- } \\
7, \mathrm{n}=2\end{array}$ & $\begin{array}{c}\text { strk_Ip, day- } \\
7, \mathrm{n}=2\end{array}$ & $\begin{array}{r}\text { strk_Ip } \\
14, \mathrm{n}\end{array}$ & $\begin{array}{c}\text { strk_Ip, day- } \\
14, \mathrm{n}=2\end{array}$ & $\begin{array}{r}\text { strk_Ip } \\
14,1\end{array}$ & \\
\hline $\begin{array}{c}\text { strk_Ct, day- } \\
7, \mathrm{n}=1\end{array}$ & $\begin{array}{c}\text { strk_Ct, day- } \\
7, \mathrm{n}=1\end{array}$ & $\begin{array}{c}\text { strk_Ct, day- } \\
7, \mathrm{n}=1\end{array}$ & $\begin{array}{c}\text { strk_Ct, day- } \\
14, \mathrm{n}=1\end{array}$ & $\begin{array}{c}\text { strk_Ct, day- } \\
14, n=1\end{array}$ & $\begin{array}{c}\text { strk_Ct, day- } \\
14, \mathrm{n}=1\end{array}$ & \multirow{2}{*}{$\begin{array}{c}\text { sham_Ip, } \\
\text { day-28, } n=4\end{array}$} \\
\hline $\begin{array}{c}\text { sham_Ip, } \\
\text { day-7, n=2 }\end{array}$ & $\begin{array}{c}\text { sham_Ip, } \\
\text { day-7, n=2 }\end{array}$ & $\begin{array}{c}\text { sham_Ip, } \\
\text { day-7, n=2 }\end{array}$ & $\begin{array}{c}\text { sham_Ip, } \\
\text { day-14, n=2 }\end{array}$ & $\begin{array}{c}\text { sham_Ip, } \\
\text { day-14, n=2 }\end{array}$ & $\begin{array}{c}\text { sham_Ip, } \\
\text { day-14, n=2 }\end{array}$ & \\
\hline
\end{tabular}

Subsequently, the resulting peptide mixtures were each fractionated by high-pH fractionation on XBridge BEH C18 column (Waters, $130 \AA$, $3.5 \mu \mathrm{m}, 2.1 \times 150 \mathrm{~mm}$ ) and concatenated into twelve fractions of similar complexities, resulting in 84 sub-samples. The peptides were desalted using C18 StageTip, speed-vac dried, and kept in $-20{ }^{\circ} \mathrm{C}$ until nanoLC-MS/MS analysis 


\section{Supplementary Figures}

Figure S1: Differentiated Proteomes in Stroke and Control Samples.

Principal component analysis of proteomes on day-1, day-3, day-7, day-14 and day-28 samples.

Figure S2. Temporal Profiles of 309 Top-Hits in Stroke and Control Samples

Protein temporal profiles in sham_Ip, strk_Ct and strk_Ip samples.

Figure S3. Western Blots of Albumin in the Brain Cortex Tissue Samples.

Western blot images of Alb in the analyzed brain cortex tissue samples. 
Figure S1 Differentiated Proteomes in Stroke and Control Samples

Principal Clustering Analysis of proteomes on day-1, day-3, day-7, day-14 and day-28 samples after DHMCAO surgery. dot color: Stroke contralateral (strk_Ct), green; Stroke ipsilateral (strk_lp), orange; Sham ipsilateral (sham_Ip), purple.
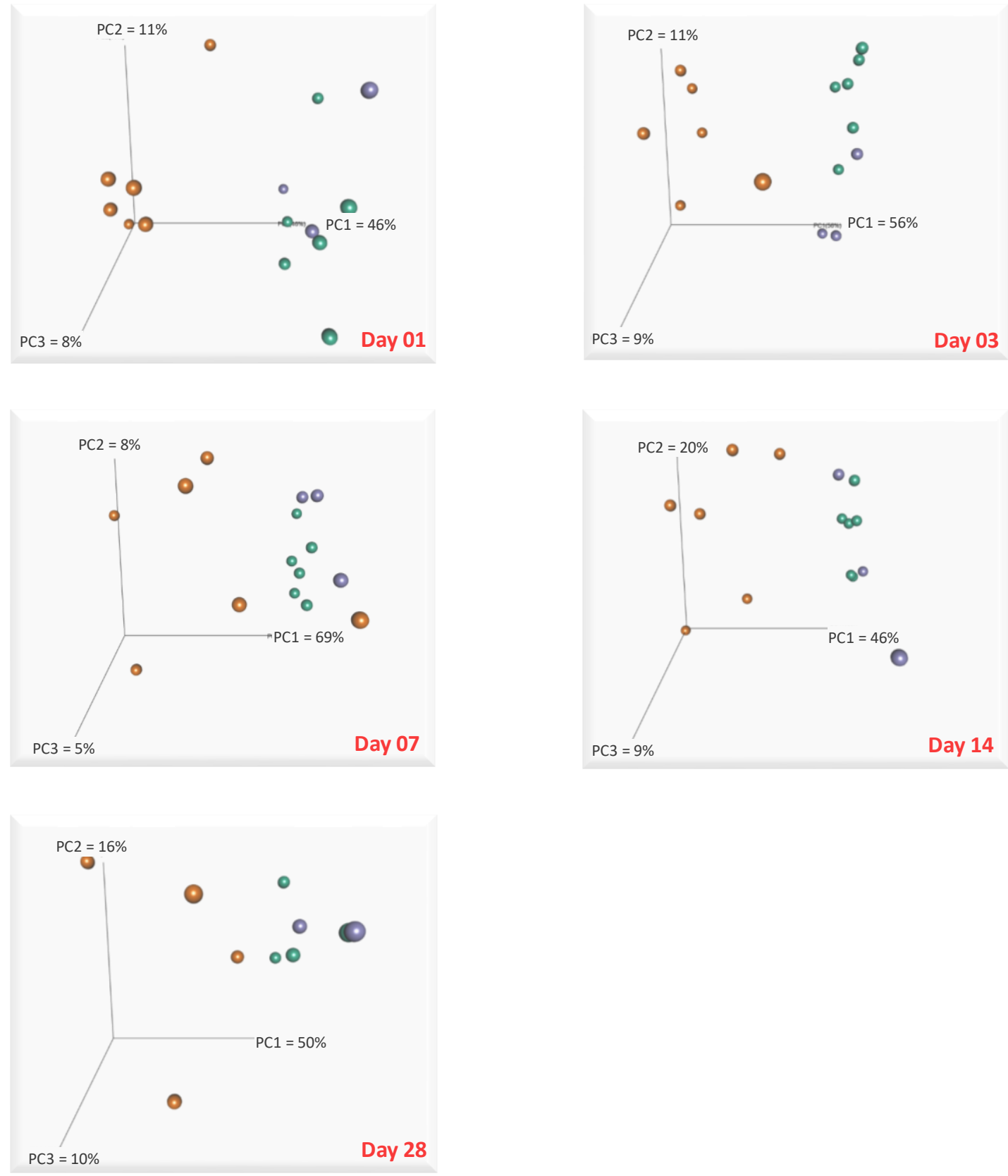
Figure S2. Temporal Profiles of 309 Top-Hits in Stroke and Control Samples.

Protein temporal profiles in sham_Ip, strk_Ct and strk_Ip samples. Data were normalized as described in the method section. $X$ axis: sample collecting day post-stroke, $Y$ axis: normalized and scaled protein expression. For each plot: red, strk_Ip; blue, sham_Ip; green, strk_Ct; diamond and dot: mean of all sample replicates; error bar: one standard deviation. 
Unique_ID=A2m_Q61838

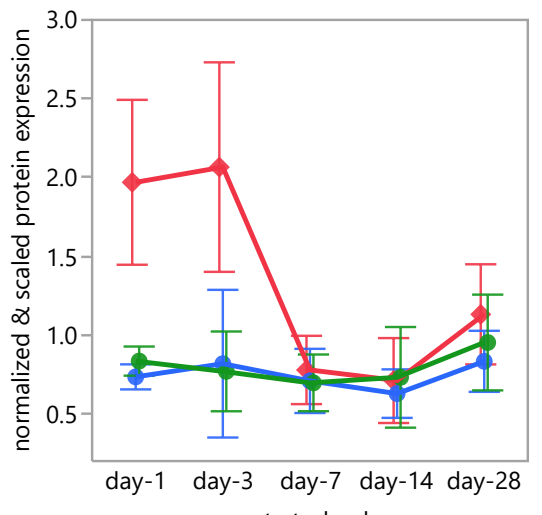
post-stroke day

Unique_ID=Agt_P11859

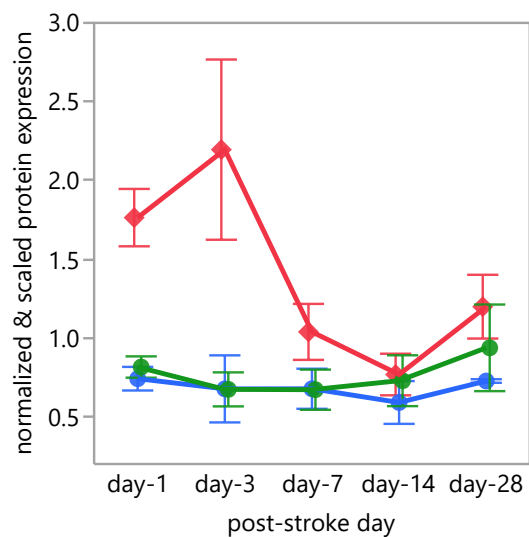

Unique_ID=Alb_P07724

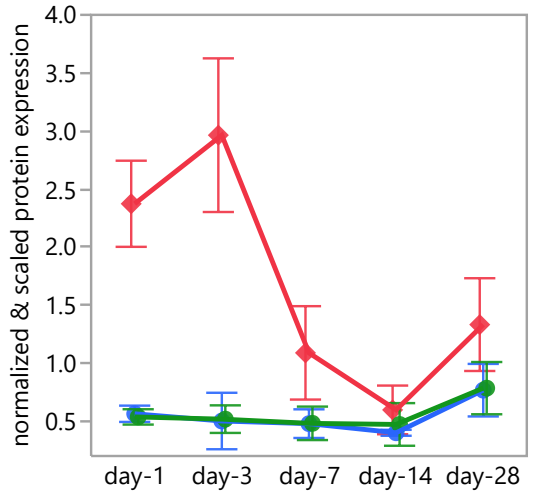

post-stroke day

Unique_ID=Anxa1_P10107

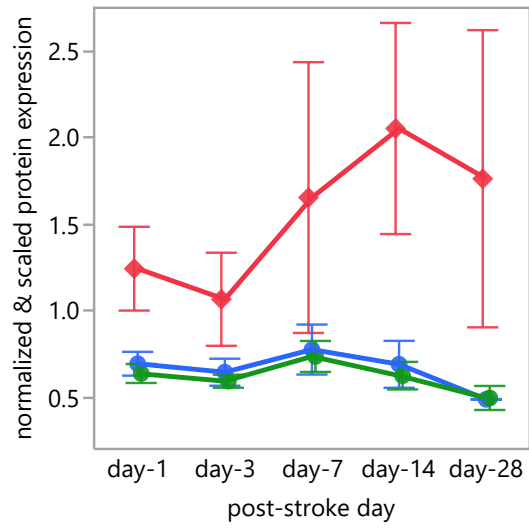

Figure_S2_309TopHits_Profile
Unique_ID=A2mp_Q6GQT1

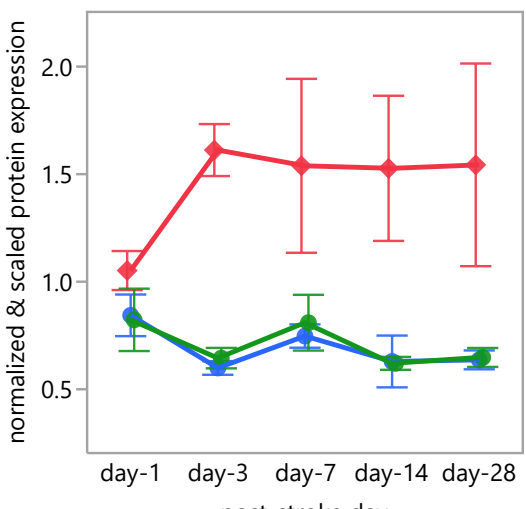

Unique_ID=Ahsg_P29699

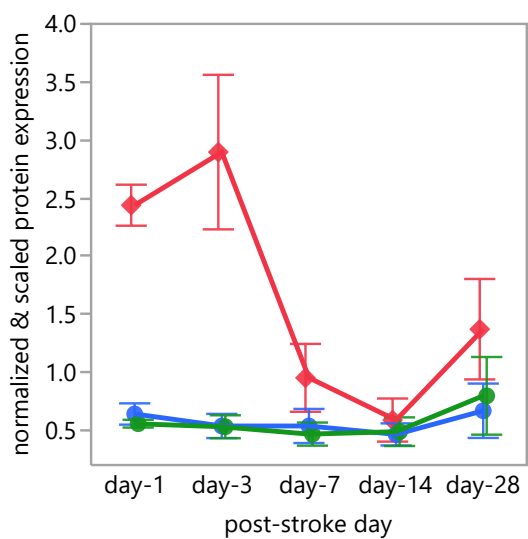

Unique_ID=Ambp_Q07456

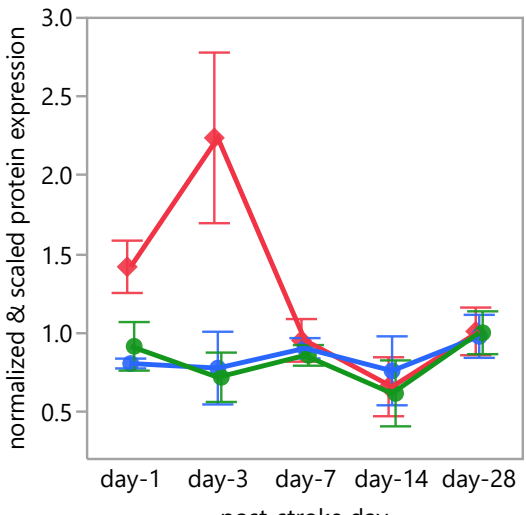

post-stroke day

Unique_ID=Anxa4_P97429

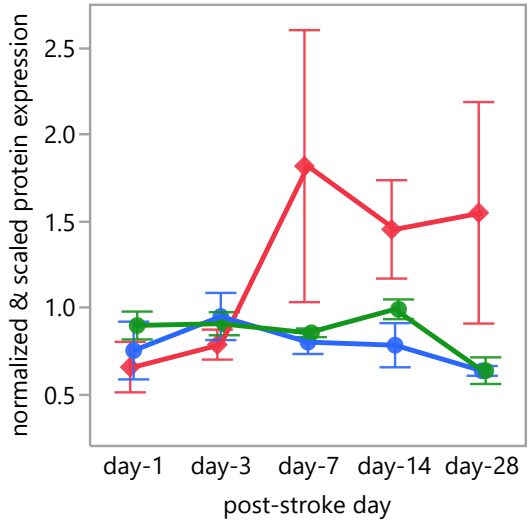

Unique_ID=Afm_089020

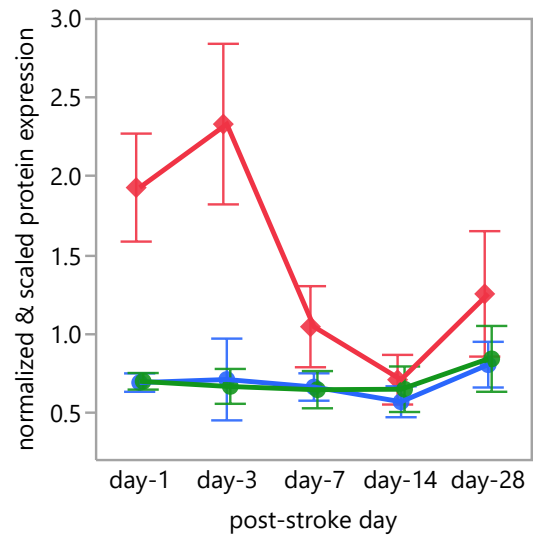

Unique_ID=Aif1_O70200

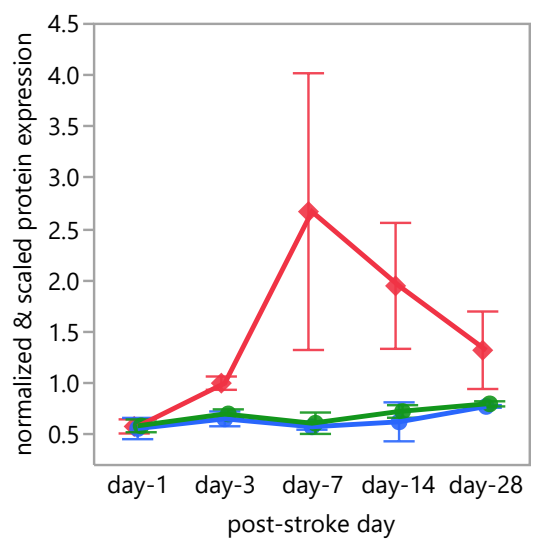

Unique_ID=Anpep_P97449

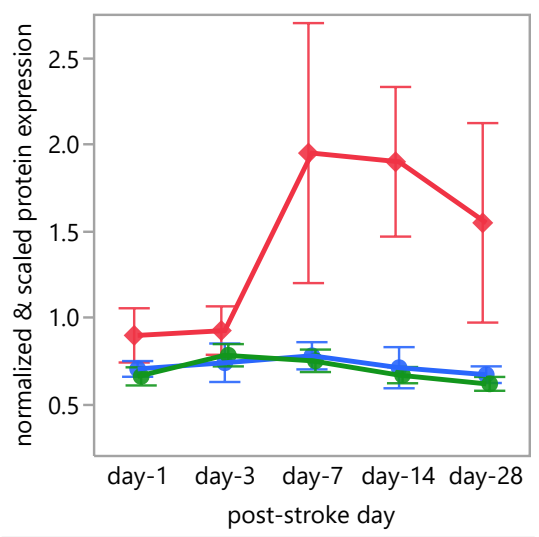

Unique_ID=Apbb1ip_Q8R5A3

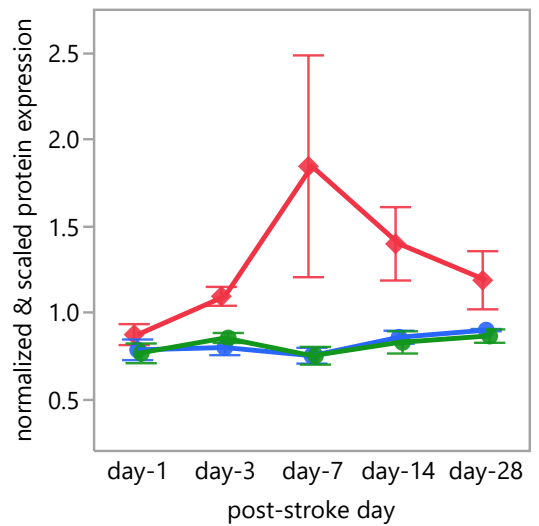


Unique_ID=Apcs_P12246

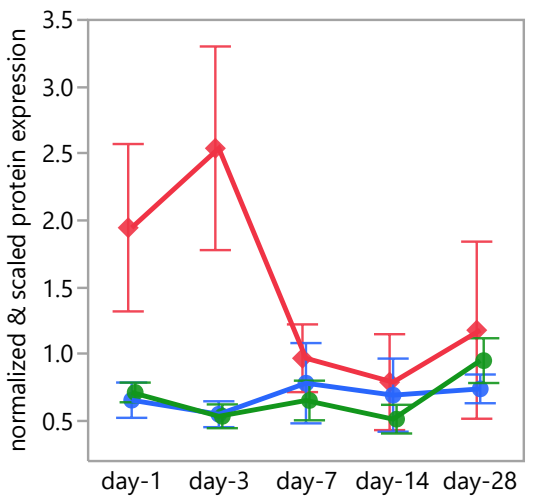

post-stroke day

Unique_ID=Apoa4_P06728

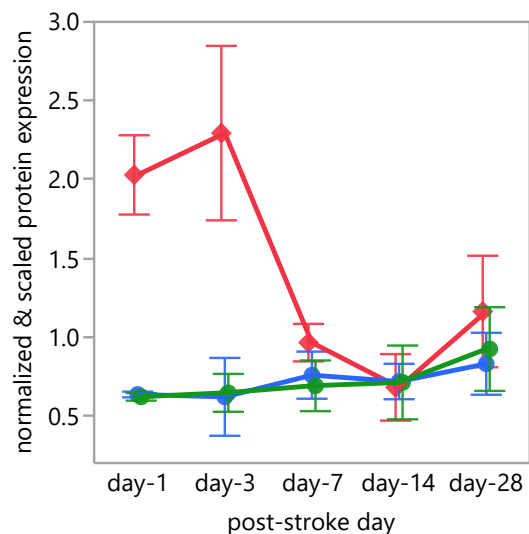

Unique_ID=Apod_P51910

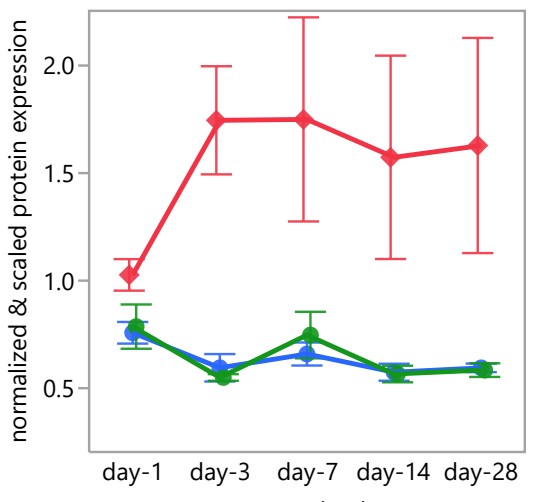

post-stroke day

Unique_ID=Arg1_Q61176

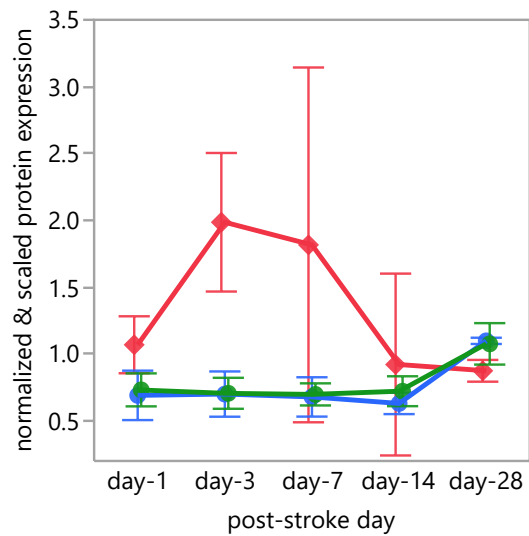

Figure_S2_309TopHits_Profile

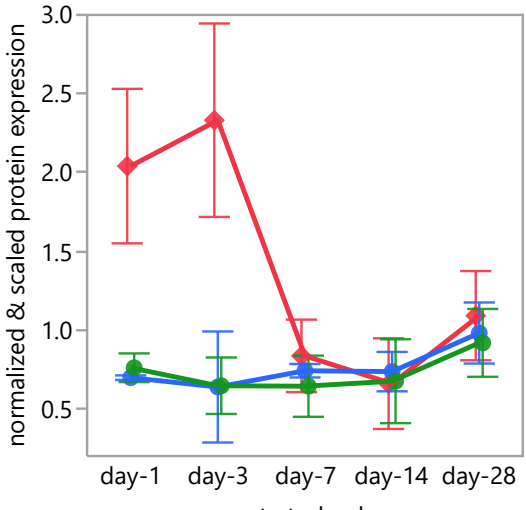

Unique_ID=Apobr_Q8VBT6

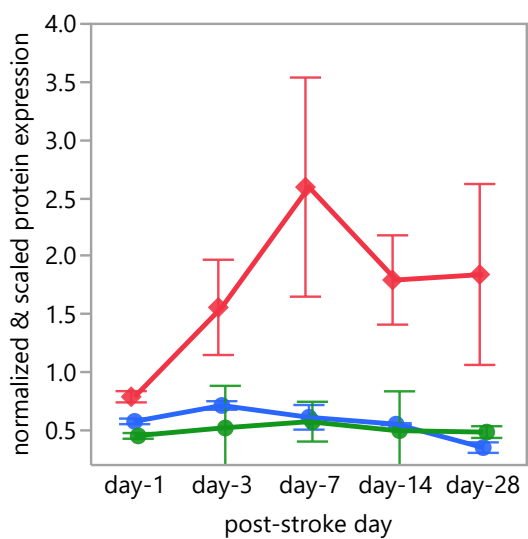

Unique_ID=Apoe_P08226

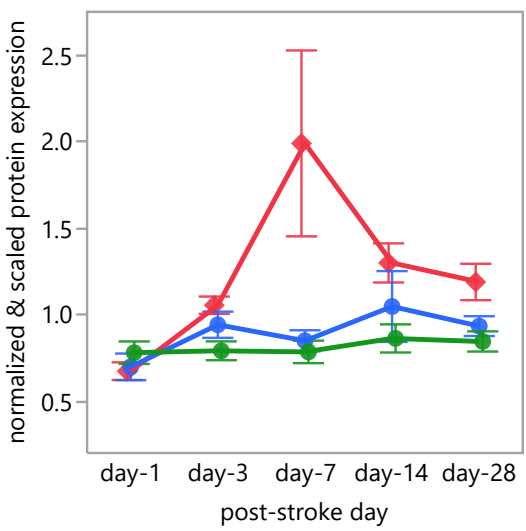

Unique_ID=Arhgdib_Q61599

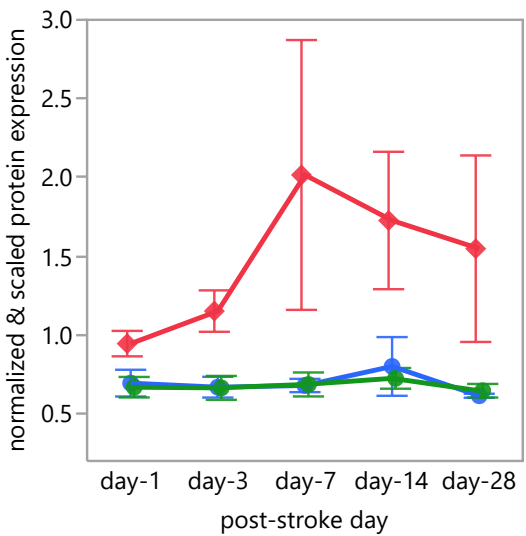

Unique_ID=Apoa2_P09813

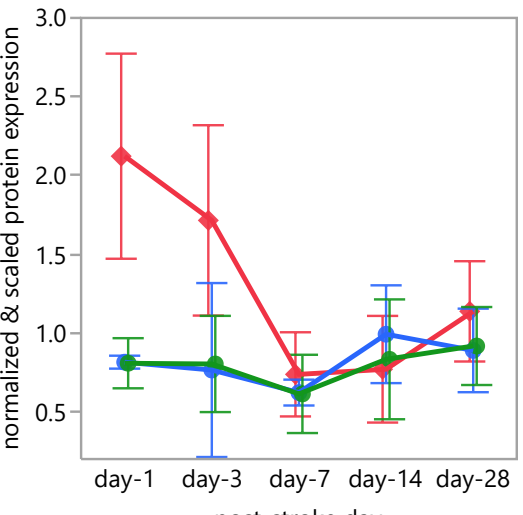
post-stroke day

\section{Unique_ID=Apoc1_P34928}

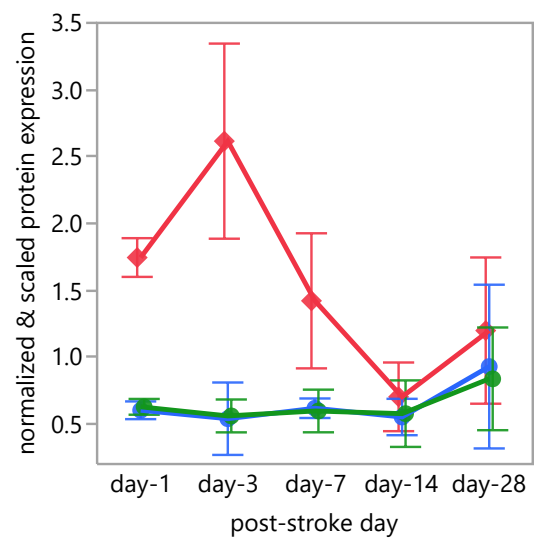

Unique_ID=Apoh_Q01339

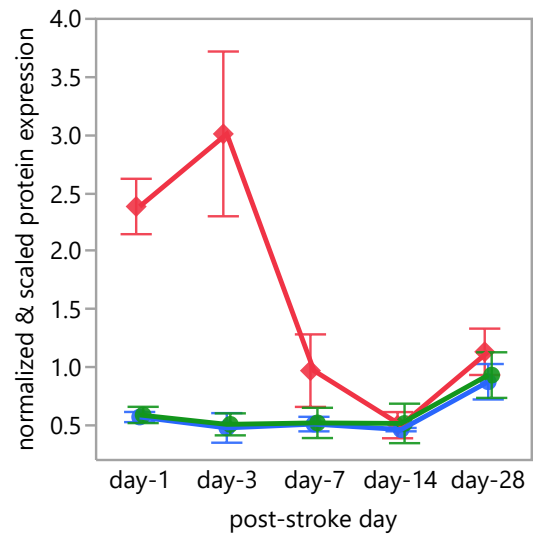

Unique_ID=Arhgef6_Q8K4I3

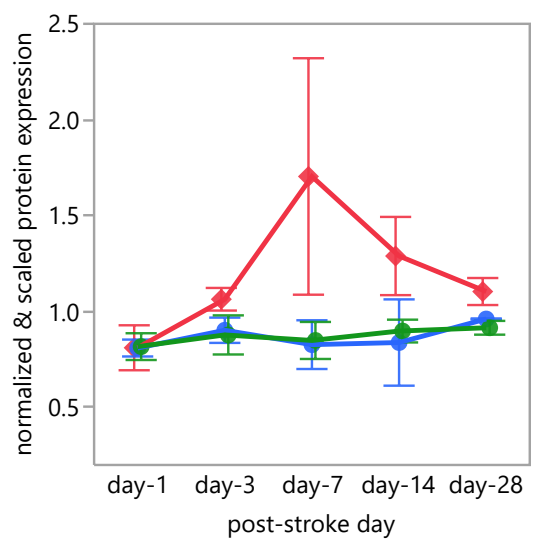


Unique_ID=Arpc1b_Q9WV32

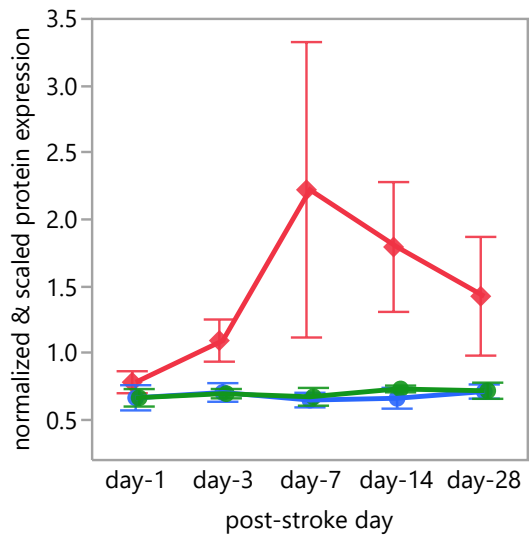

Unique_ID=Bgn_P28653

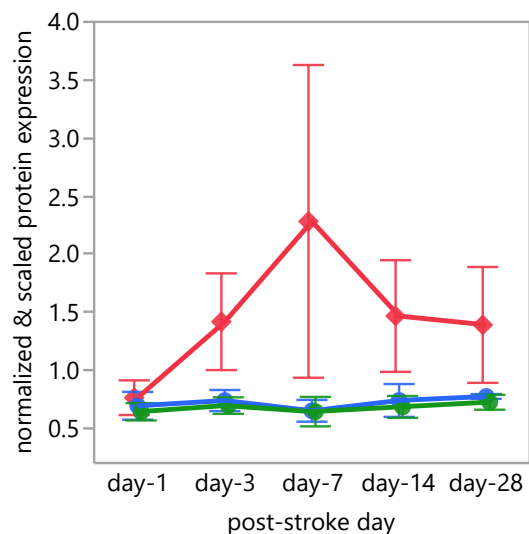

Unique_ID=Btk_P35991

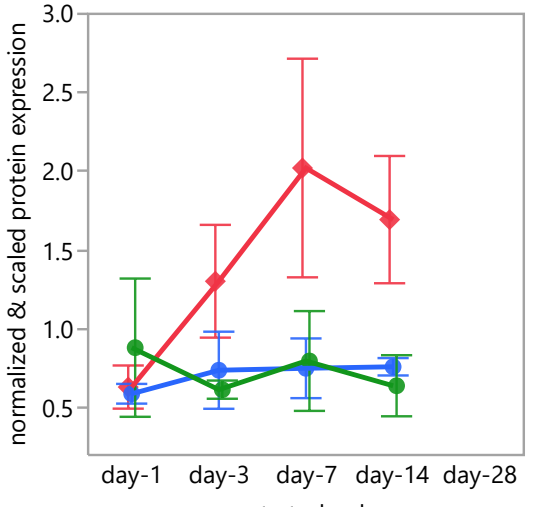

post-stroke day

Unique_ID=C1qc_Q02105

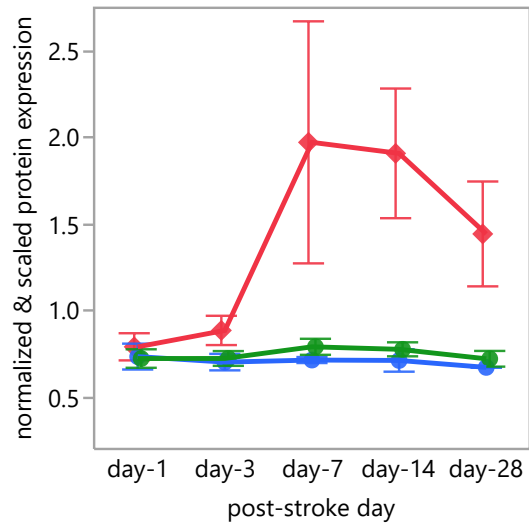

Figure_S2_309TopHits_Profile

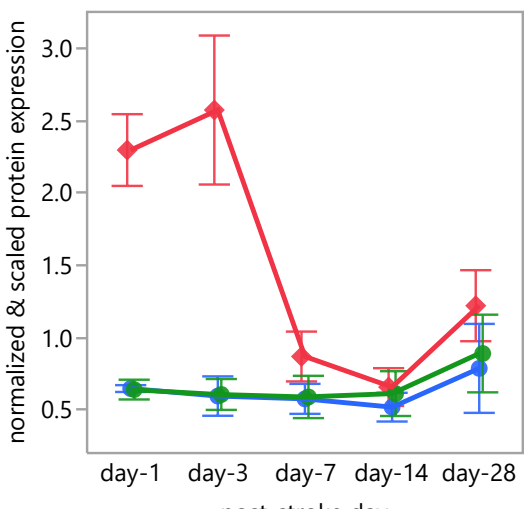

\section{Unique_ID=Bin2_D3Z6Q9}

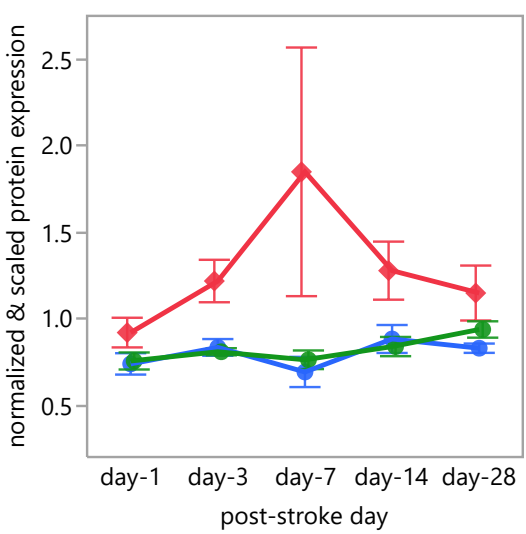

Unique_ID=C1qa_P98086

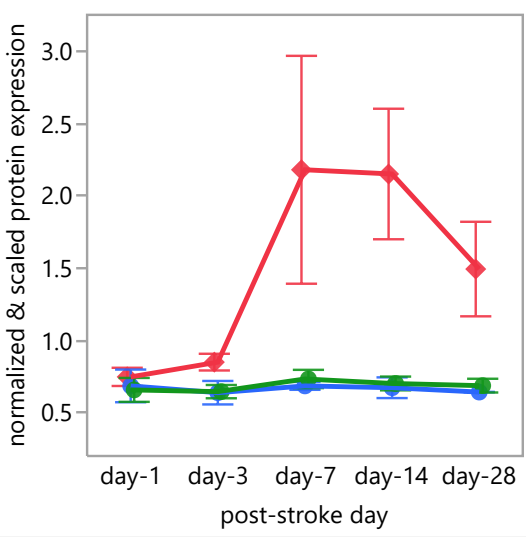

Unique_ID=C2cd3_Q52KB6-3

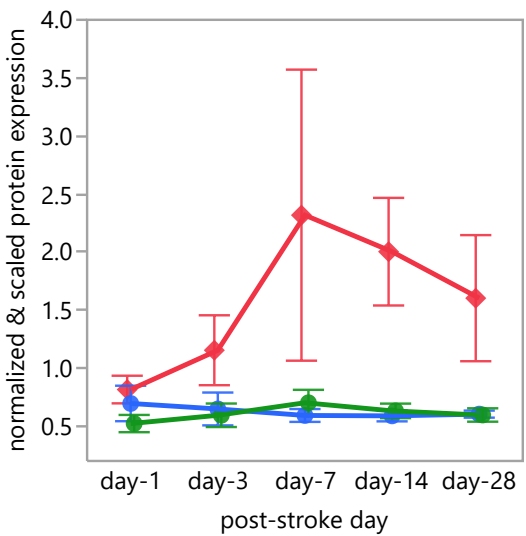

Unique_ID=B2m_P01887

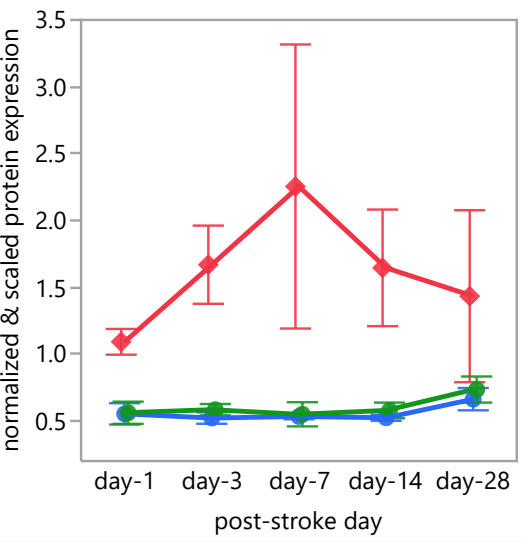

Unique_ID=Bst2_Q8R2Q8

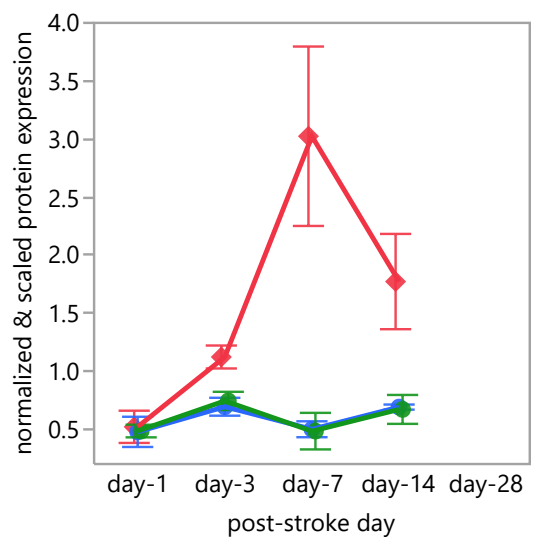

Unique_ID=C1qb_P14106

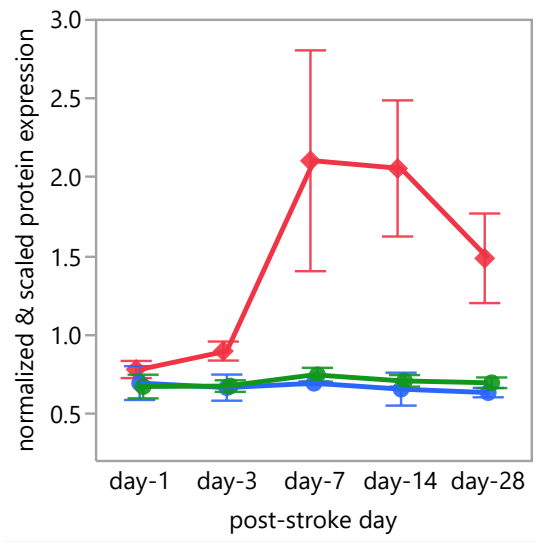

Unique_ID=C3_P01027

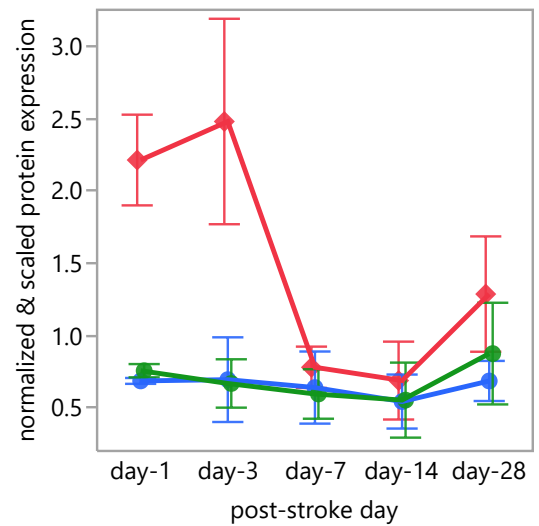


Unique_ID=C4b_P01029

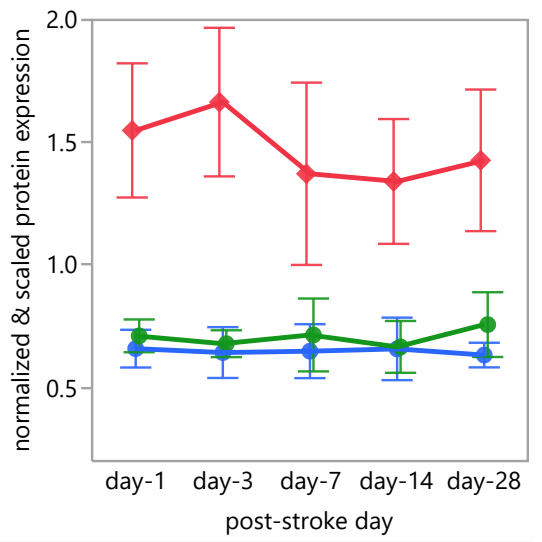

Unique_ID=C8a_Q8K182

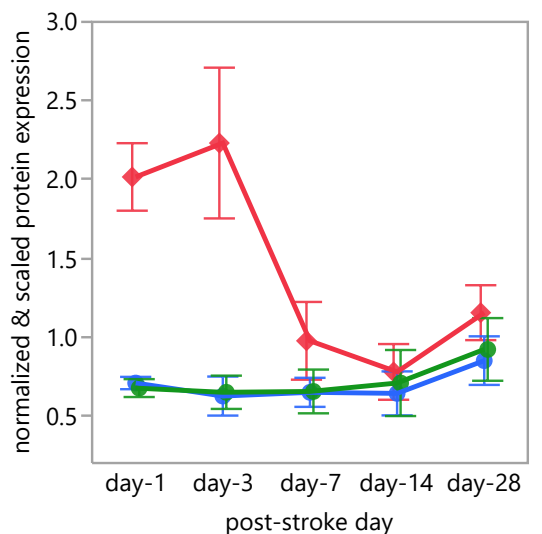

Unique_ID=C9_P06683

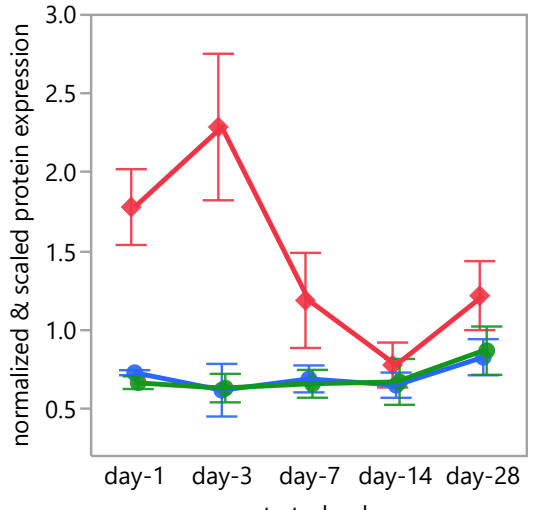

Unique_ID=Cd14_P10810

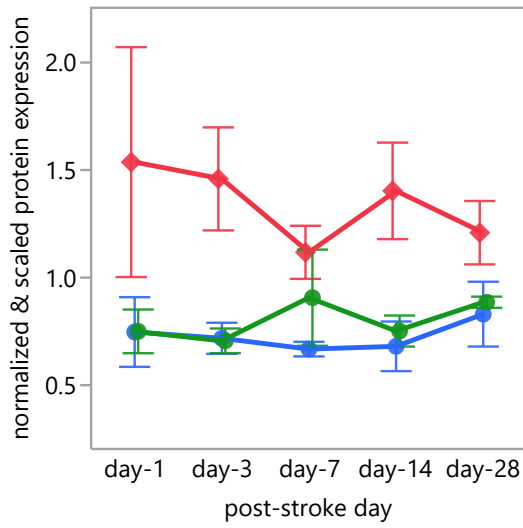

Figure_S2_309TopHits_Profile

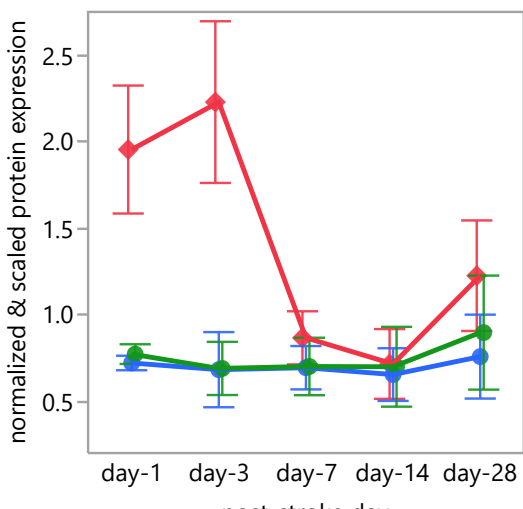

Unique_ID=C8b_Q8BH35

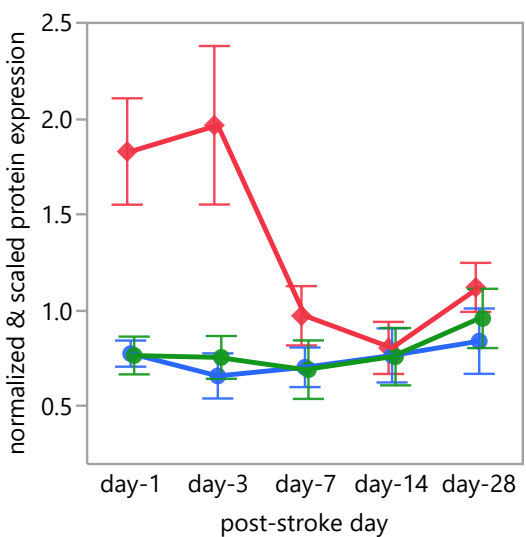

Unique_ID=Ca13_Q9D6N1

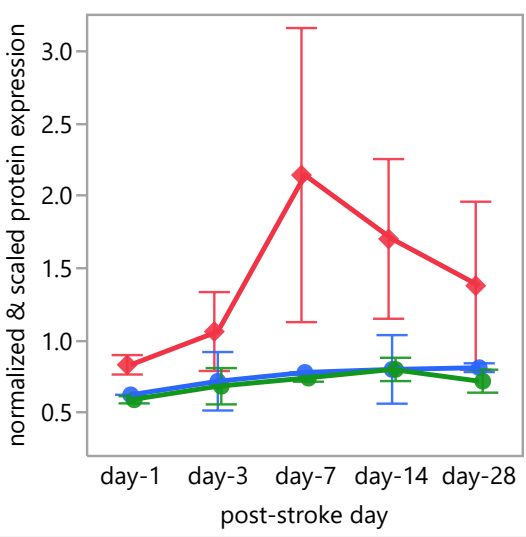

Unique_ID=Cd180_Q62192

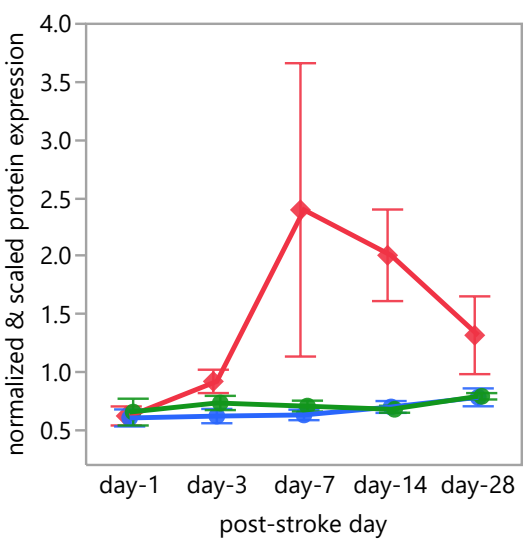

Unique_ID=C5ar1_P30993

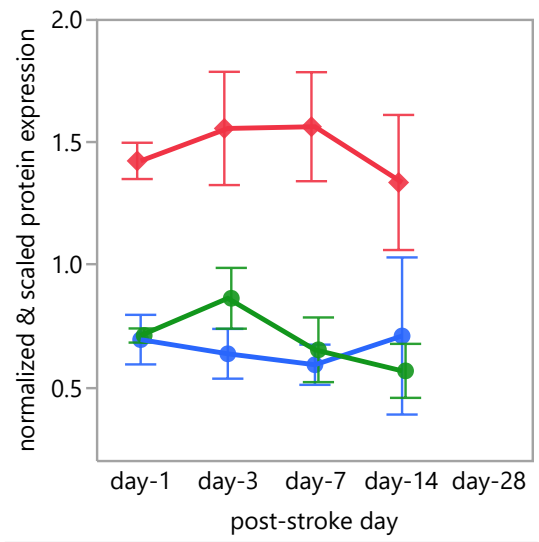

Unique_ID=C8g_Q8VCG4

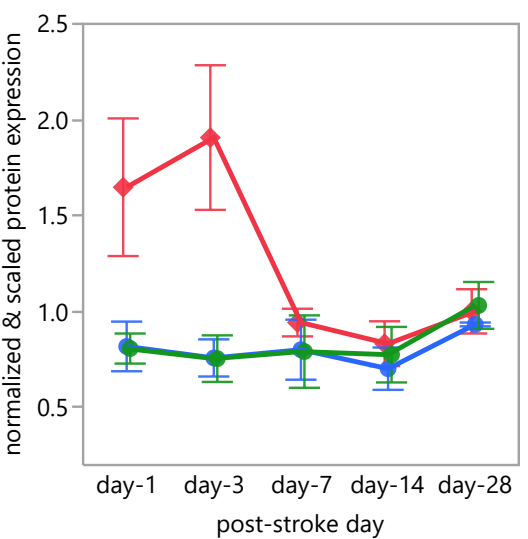

Unique_ID=Capg_P24452

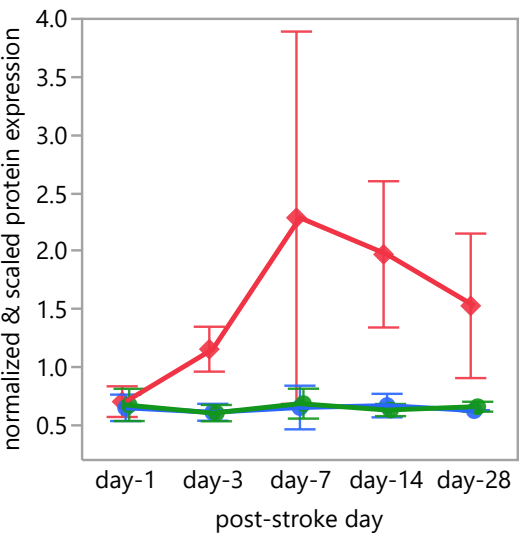

Unique_ID=Cd44_P15379-2

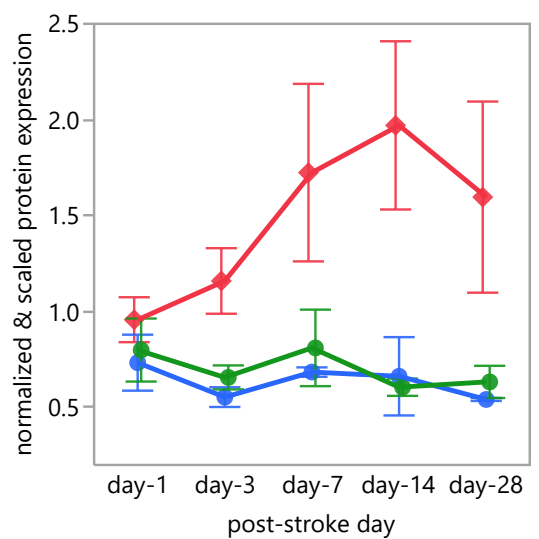




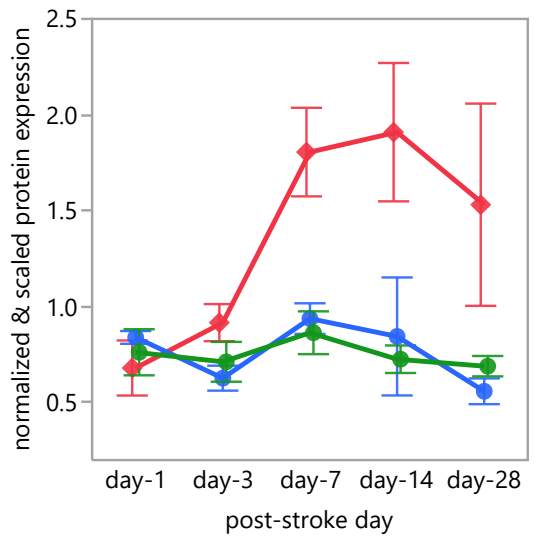

Unique_ID=Cd68_P31996-2

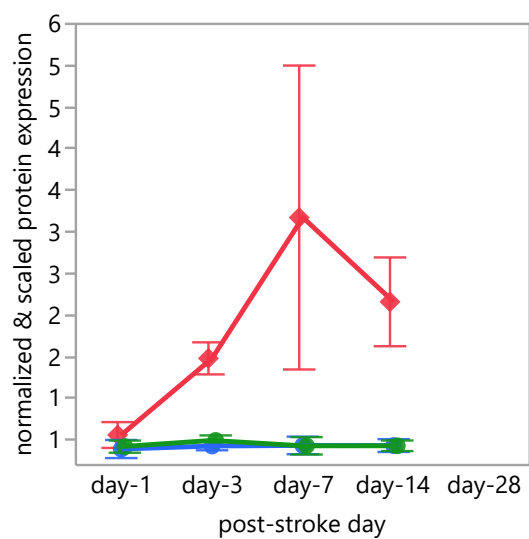

Unique_ID=Cfb_P04186

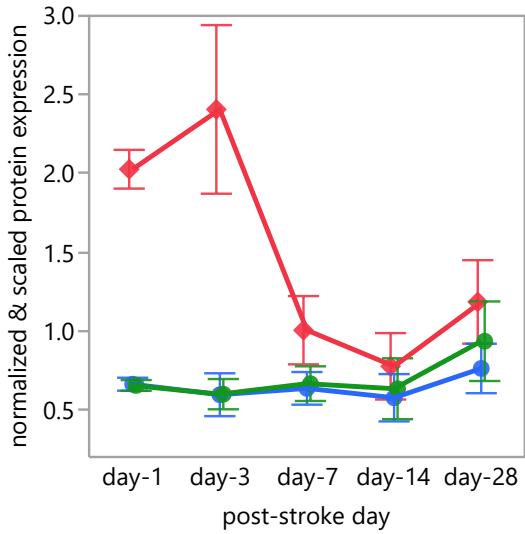

Unique_ID=Cfi_Q61129

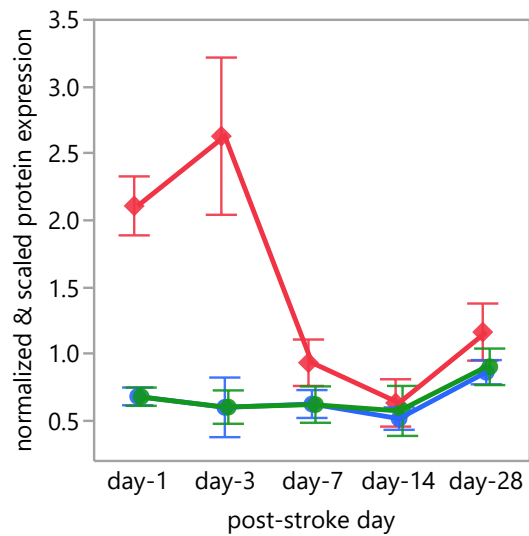

Figure_S2_309TopHits_Profile

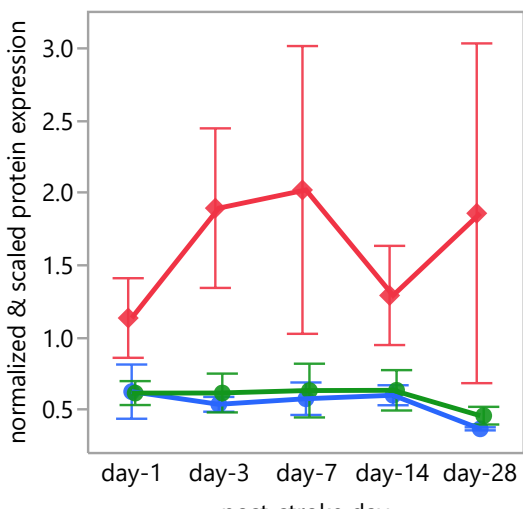

post-stroke day

\section{Unique_ID=Cd9_P40240}

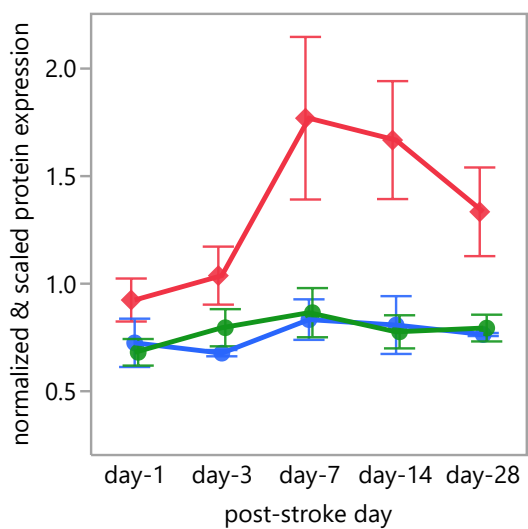

Unique_ID=Cfd_P03953-2

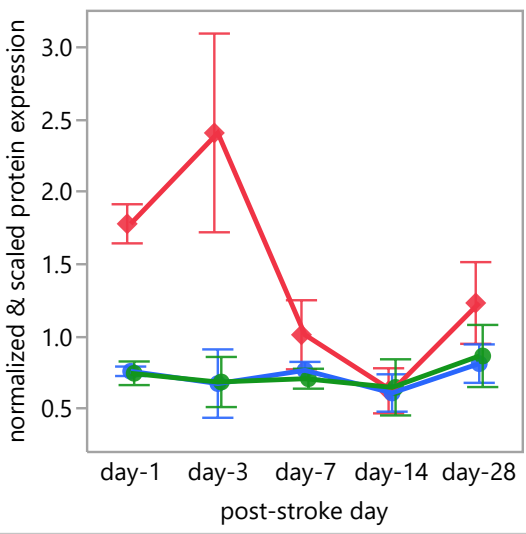

Unique_ID=Cfp_P11680

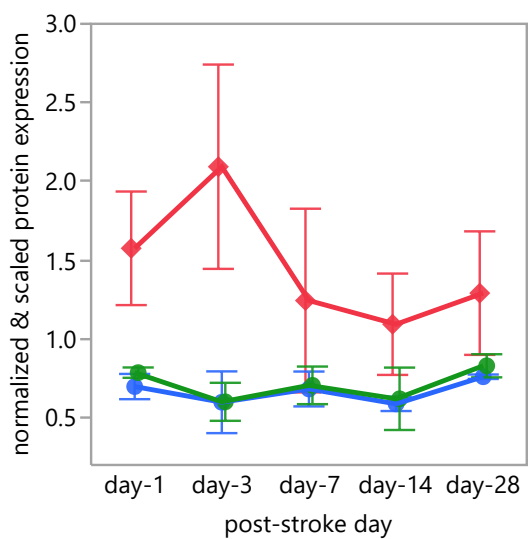

Unique_ID=Cd63_P41731

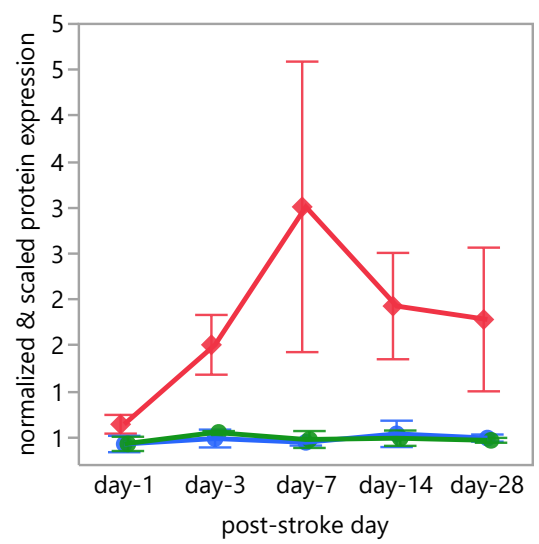

Unique_ID=Ces1c_P23953

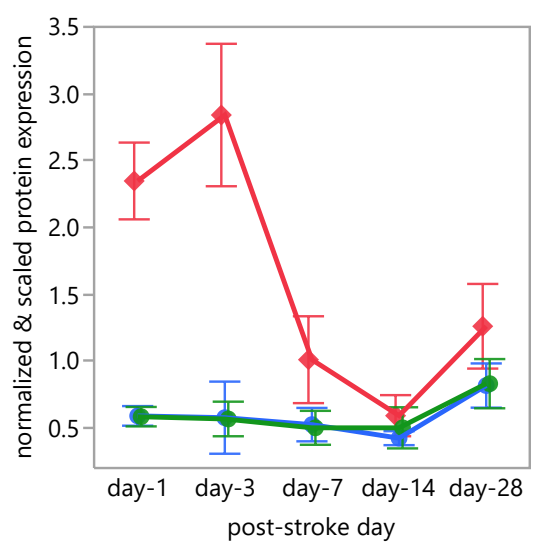

Unique_ID=Cfh_P06909

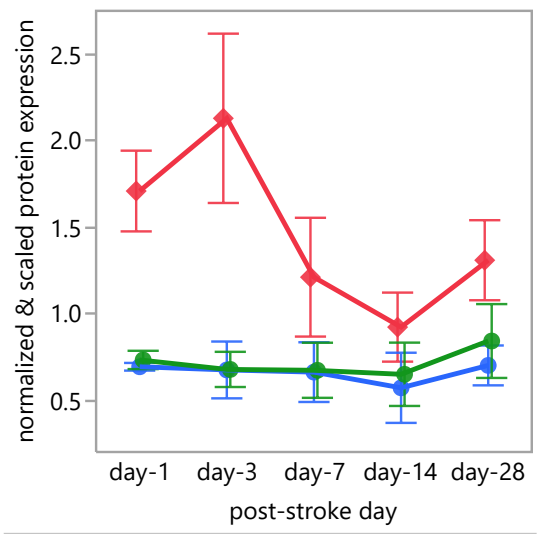

Unique_ID=Chil3_035744

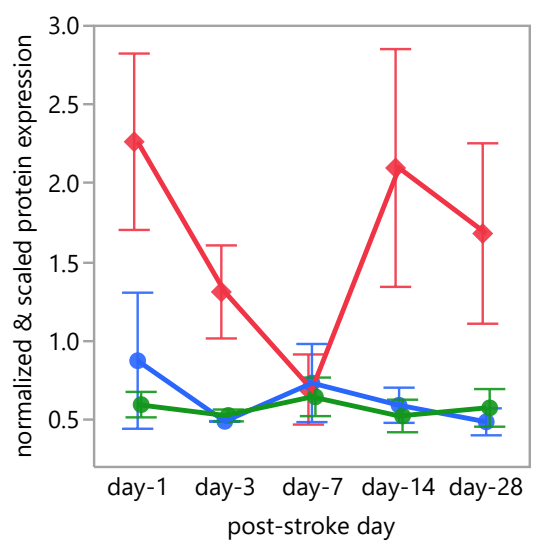




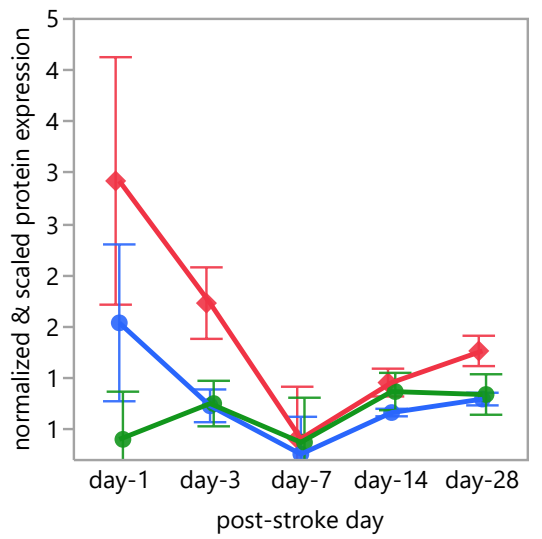

Unique_ID=Cp_Q61147

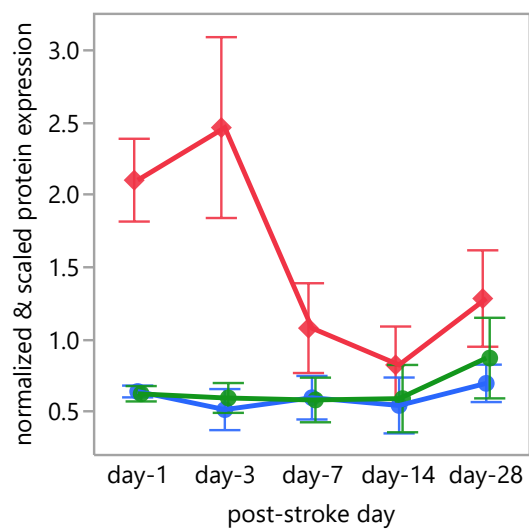

Unique_ID=Cpn2_Q9DBB9

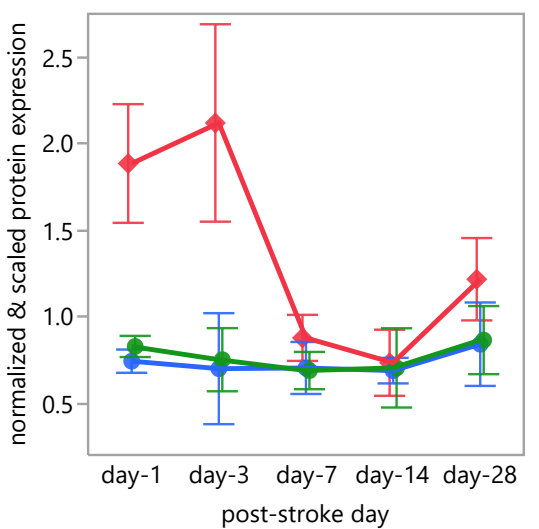

Unique_ID=Csf1r_P09581

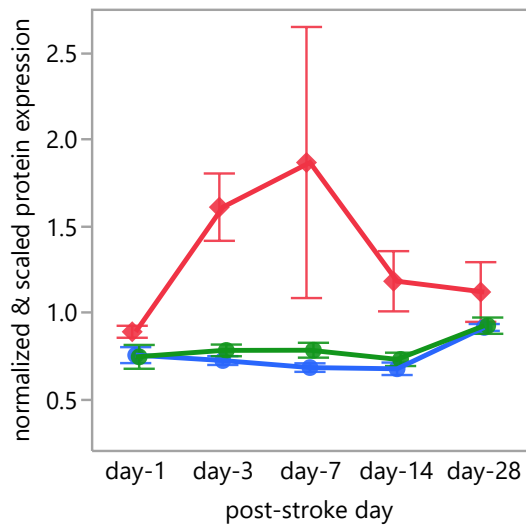

Figure_S2_309TopHits_Profile

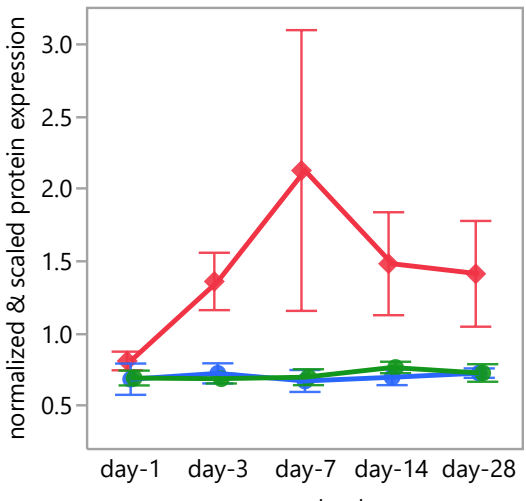

post-stroke day

\section{Unique_ID=Cpb2_Q9JHH6}

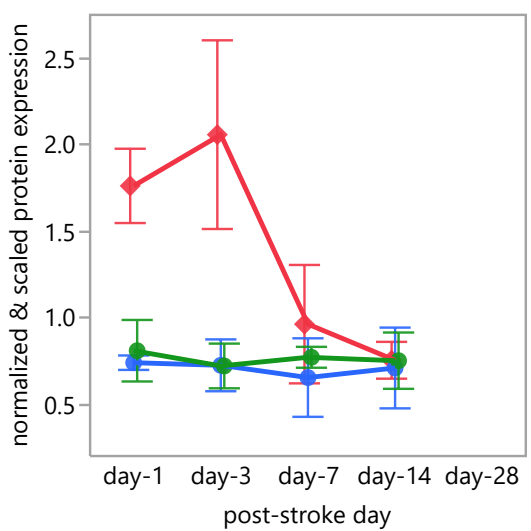

Unique_ID=Crabp1_P62965

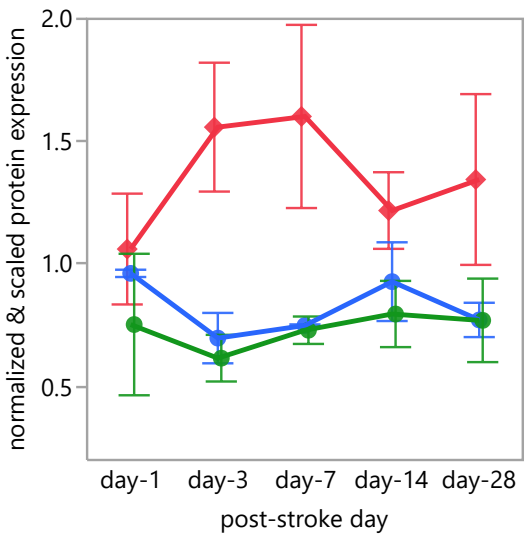

Unique_ID=Ctc1_Q5SUQ9-2

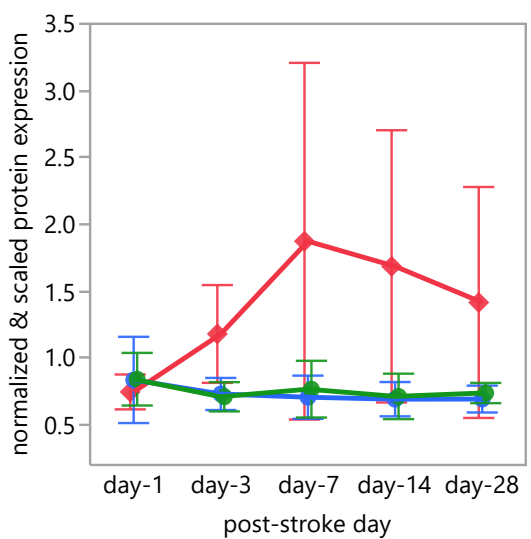

Unique_ID=Cotl1_Q9CQI6

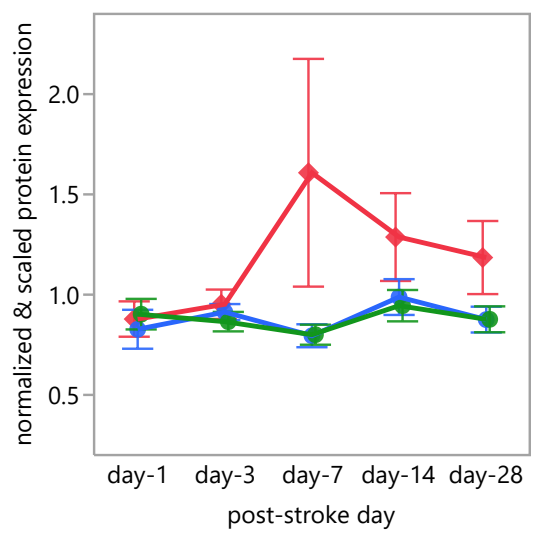

Unique_ID=Cpn1_Q9JJN5

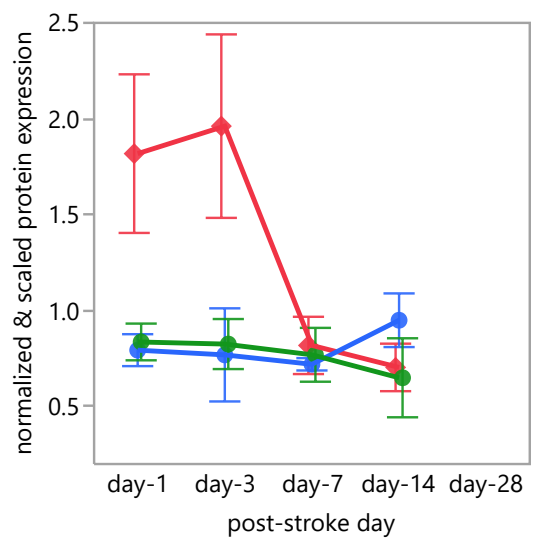

Unique_ID=Creg1_088668

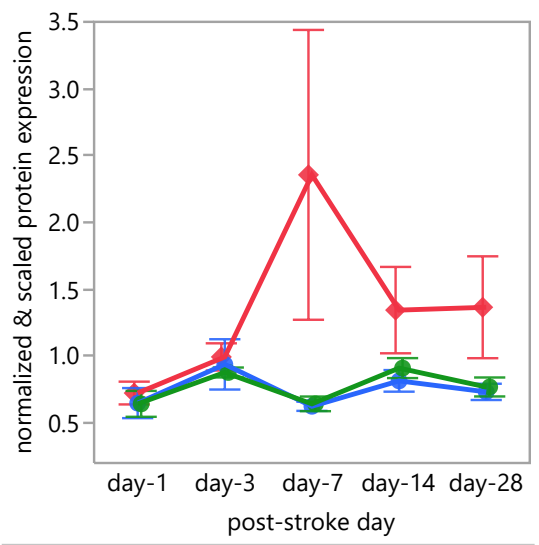

Unique_ID=Ctla2a_P12399

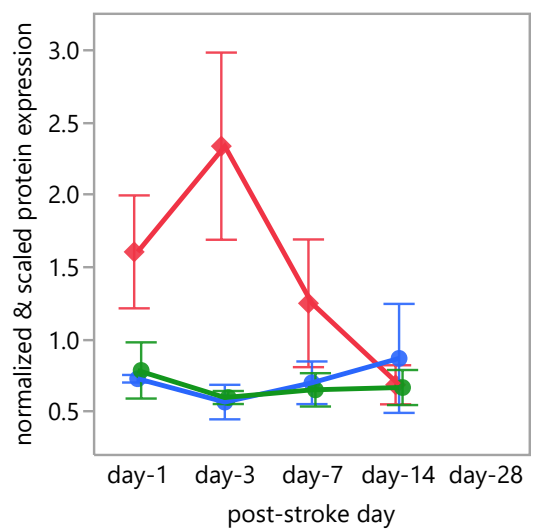




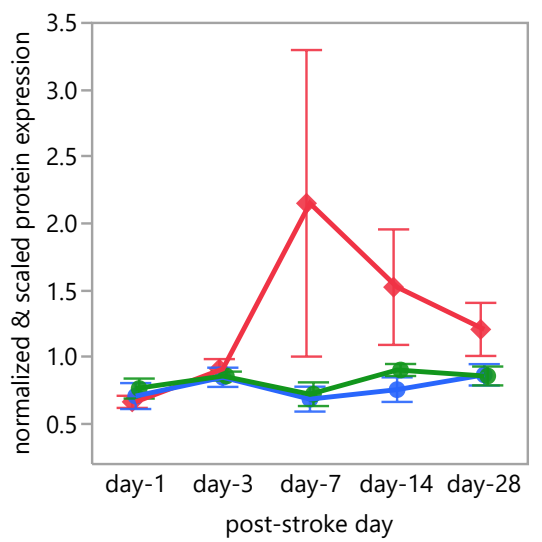

Unique_ID=Ctsd_P18242

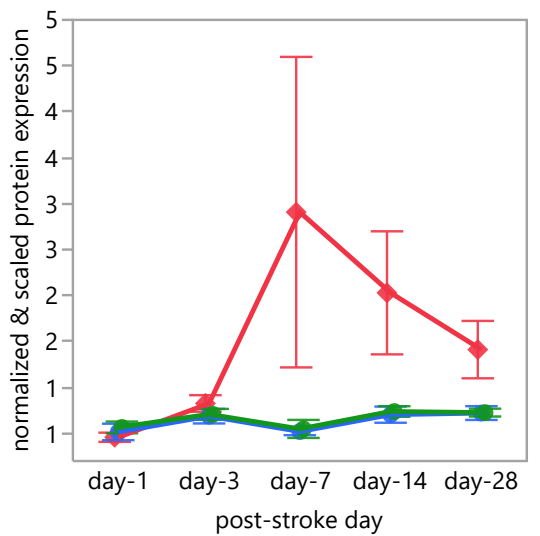

Unique_ID=Ctsz_Q9WUU7

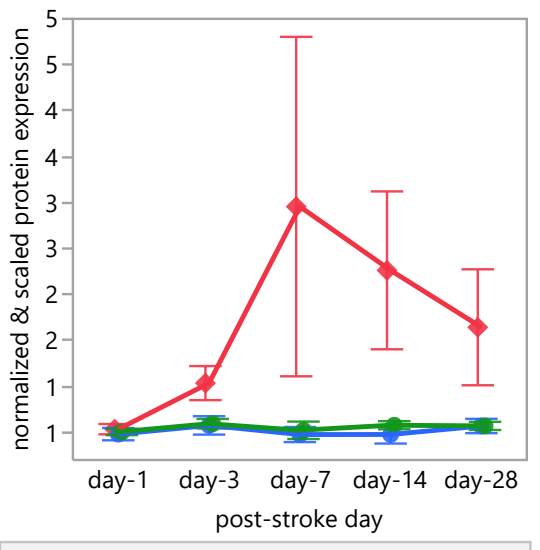

Unique_ID=Ddx58_Q6Q899

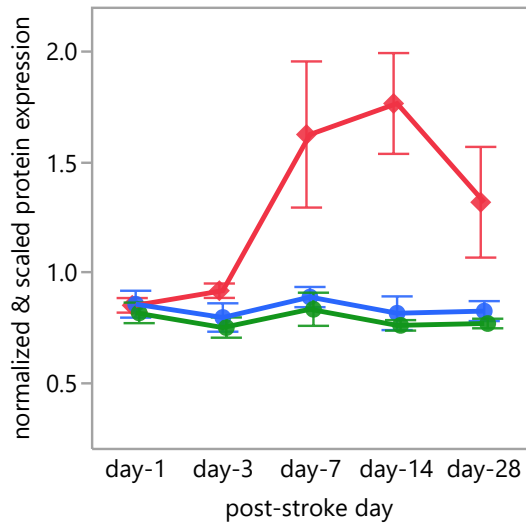

Figure_S2_309TopHits_Profile

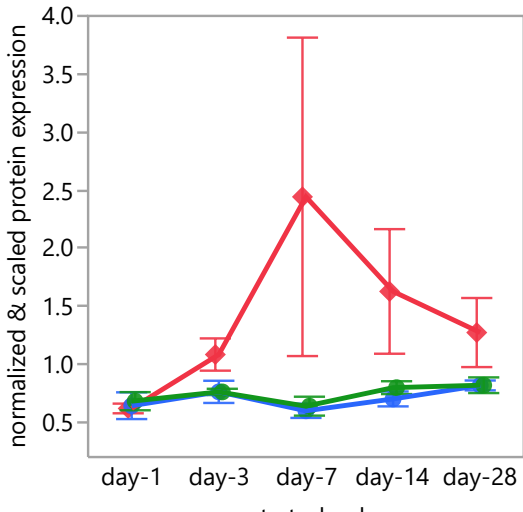

post-stroke day

\section{Unique_ID=Ctsh_P49935}

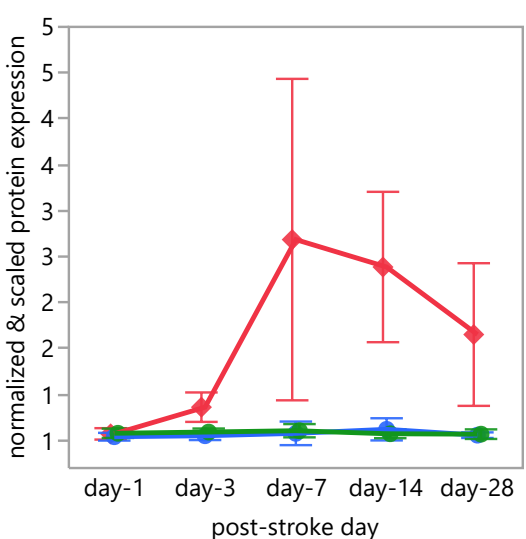

Unique_ID=Cybb_Q61093

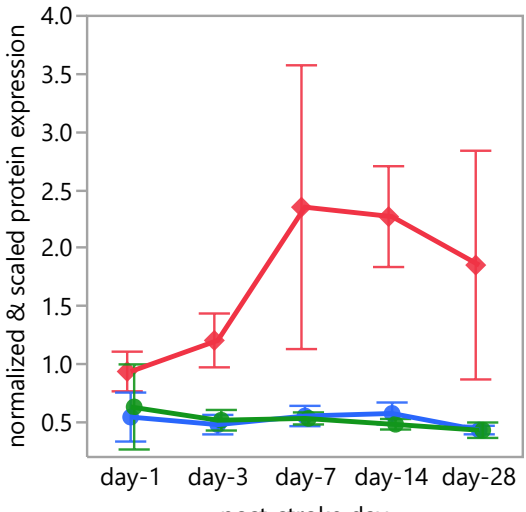

post-stroke day

Unique_ID=Dhrs1_Q99L04

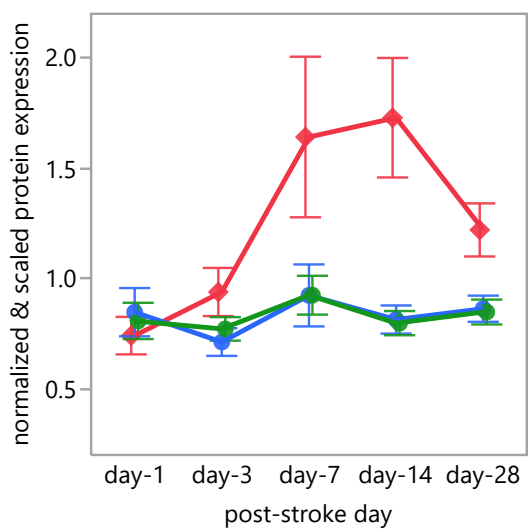

Unique_ID=Ctsc_P97821

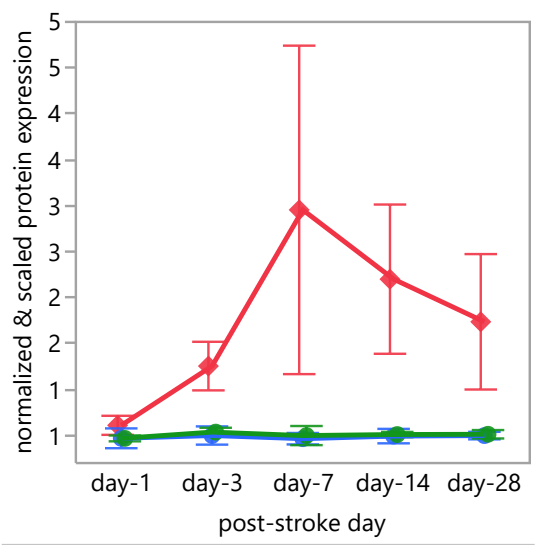

Unique_ID=Ctss_070370

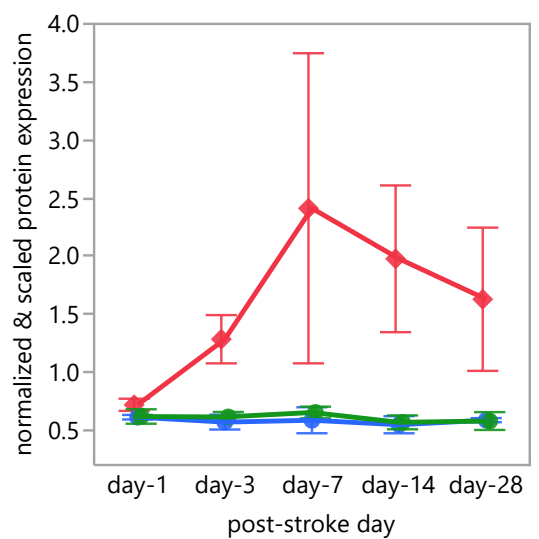

Unique_ID=Dab2_P98078-2

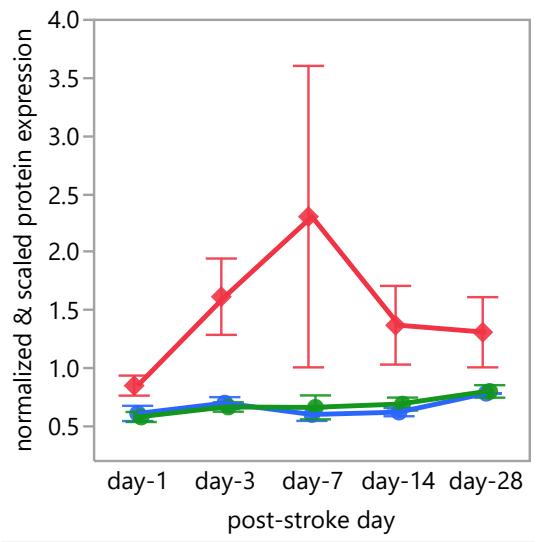

Unique_ID=Dtx3I_Q3UIR3-2

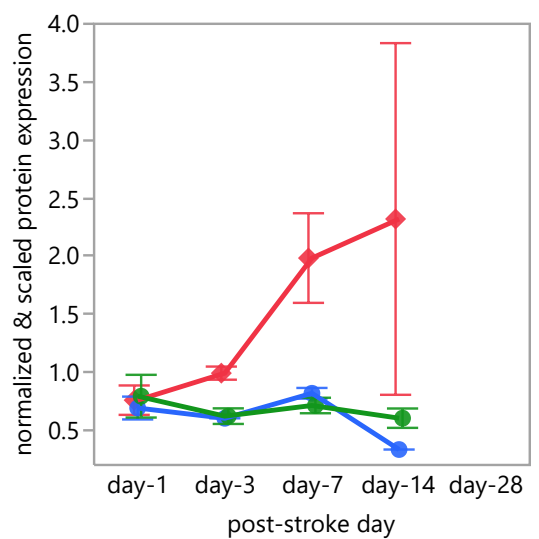




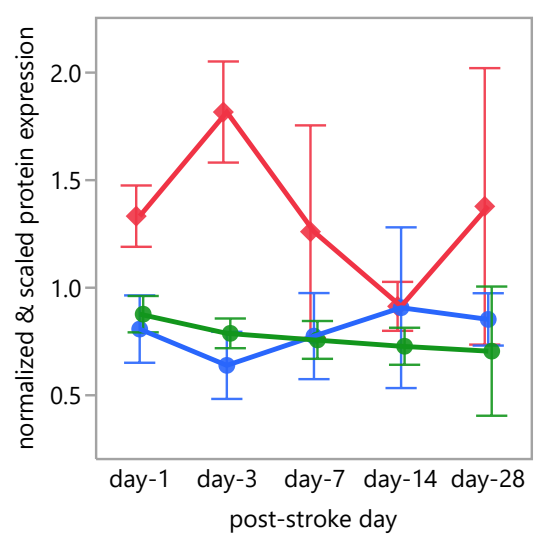

Unique_ID=Elane_Q3UP87

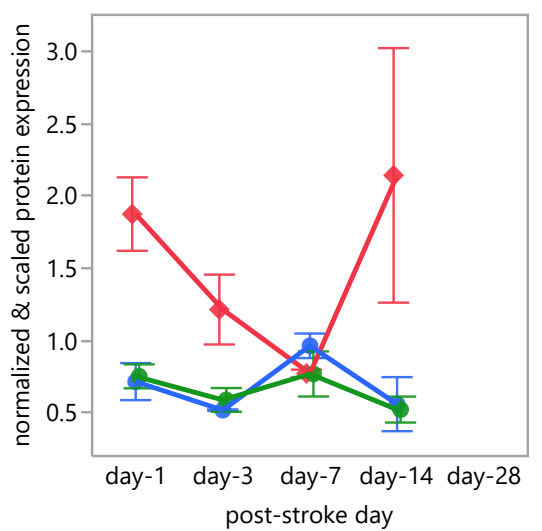

Unique_ID=F13a1_Q8BH61

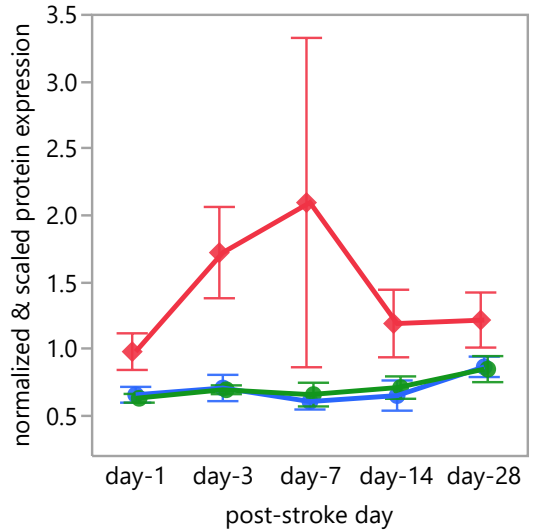

Unique_ID=Fabp4_P04117

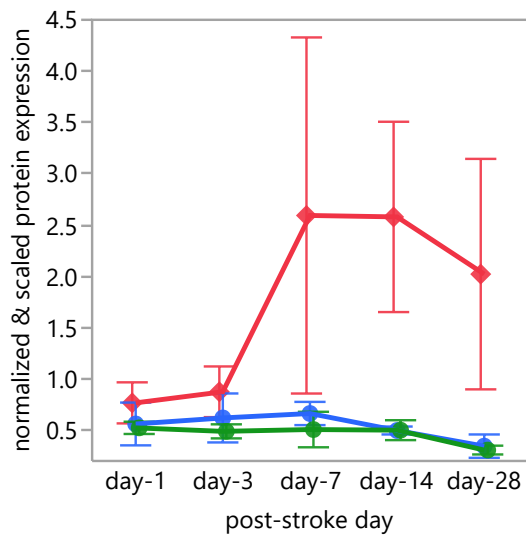

Figure_S2_309TopHits_Profile

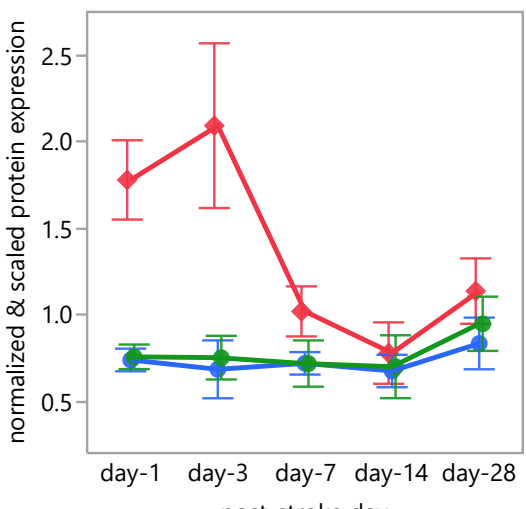

\section{Unique_ID=Erap1_Q9EQH2}

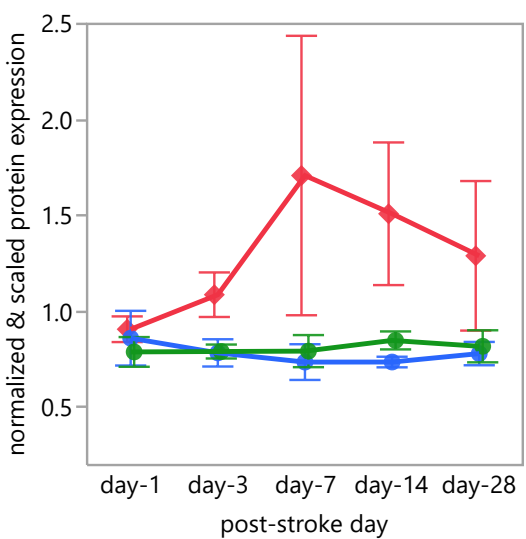

Unique_ID=F13b_Q07968

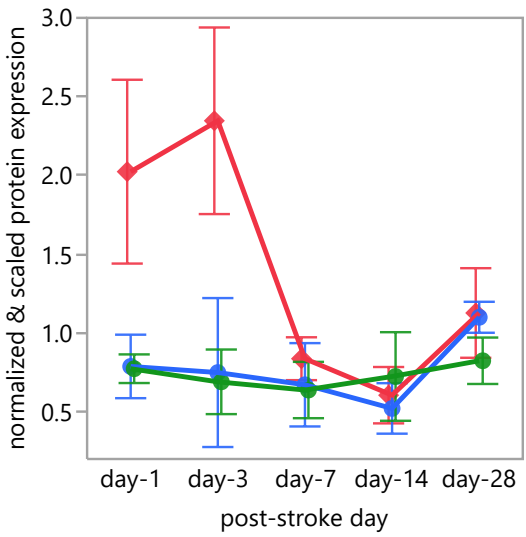

Unique_ID=Fabp7_P51880

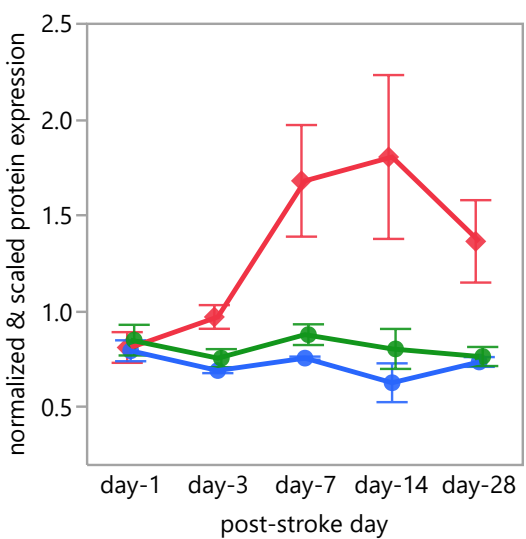

Unique_ID=Eif2ak2_Q03963

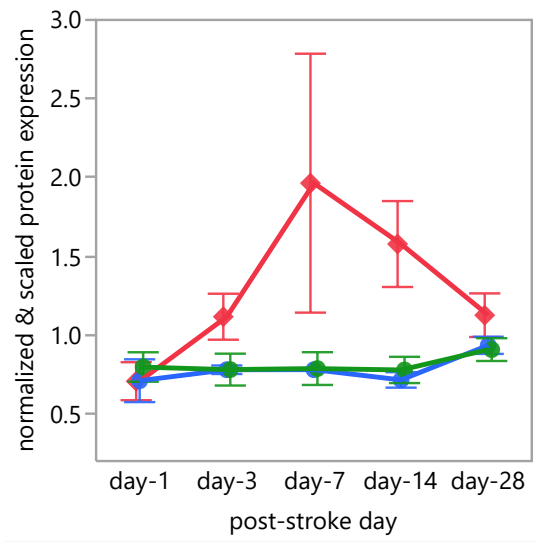

Unique_ID=F12_Q80YC5

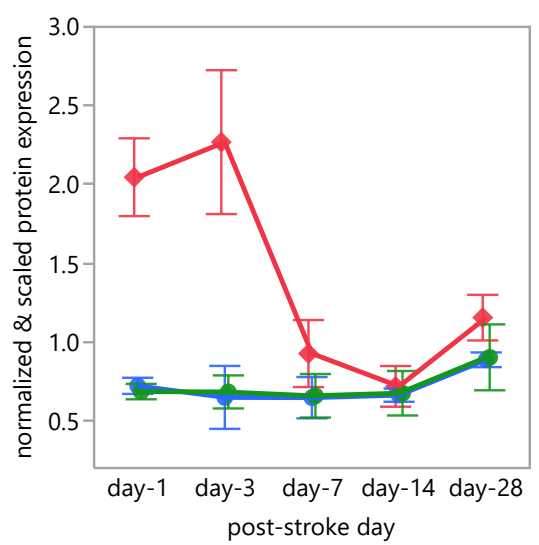

Unique_ID=F2_P19221

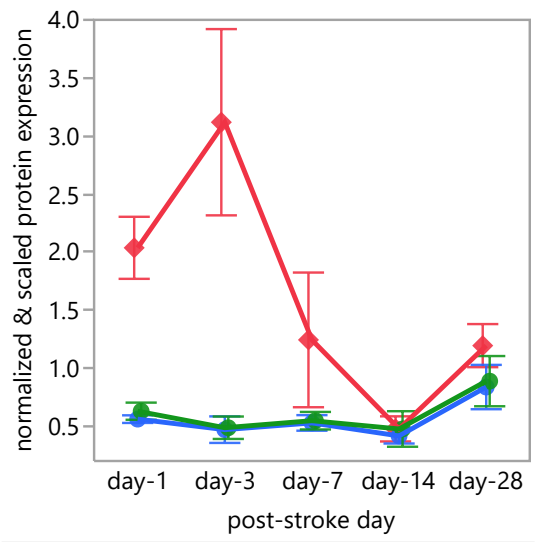

Unique_ID=Fbln1_Q08879

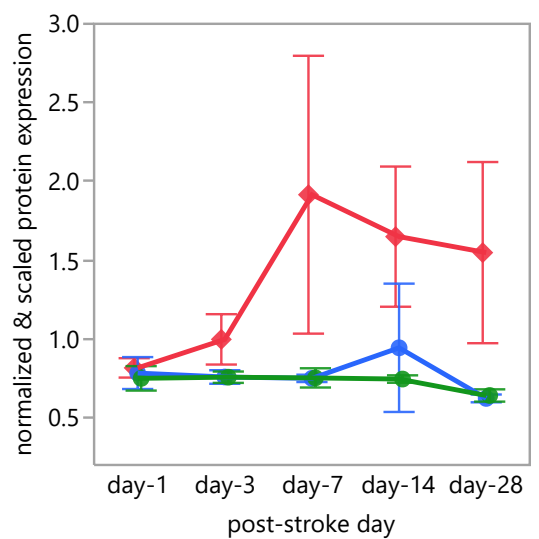


Unique_ID=Fbln1_Q08879-2

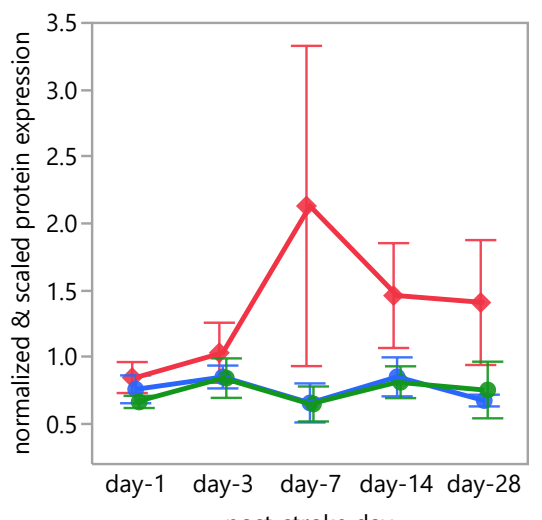

post-stroke day

\section{Unique_ID=Fcgr2_P08101-2}

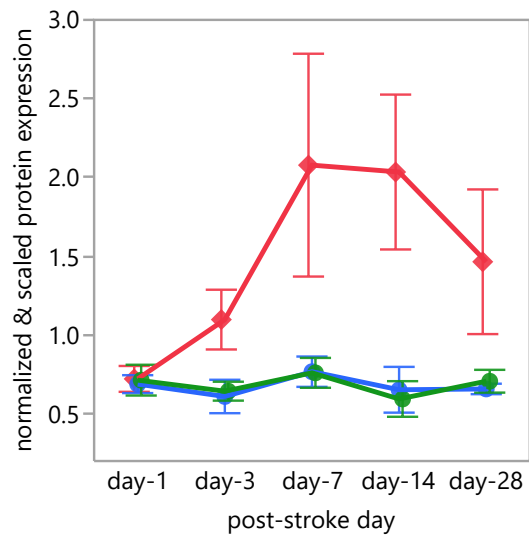

Unique_ID=Fga_E9PV24-2

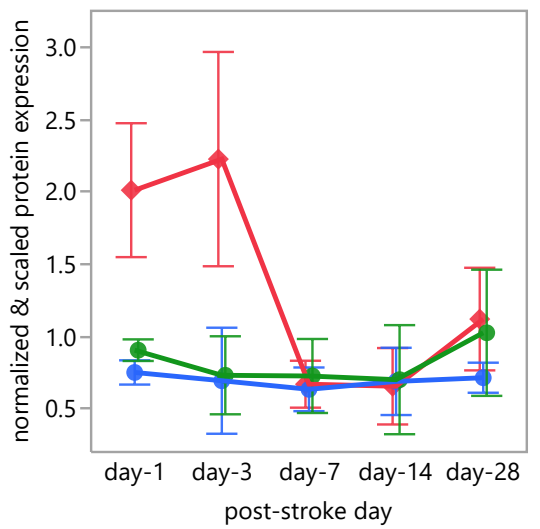

Unique_ID=FhI3_Q9R059

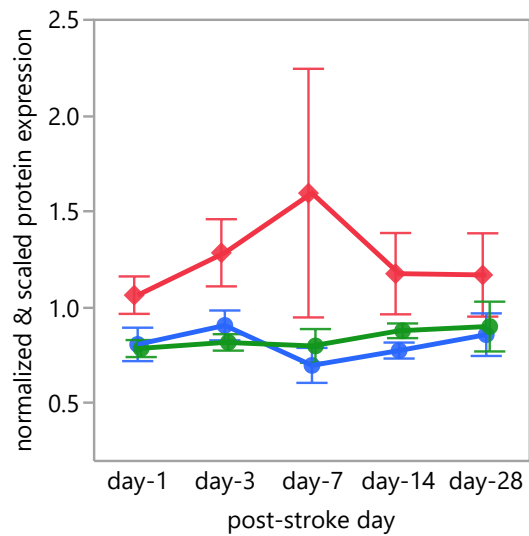

Figure_S2_309TopHits_Profile

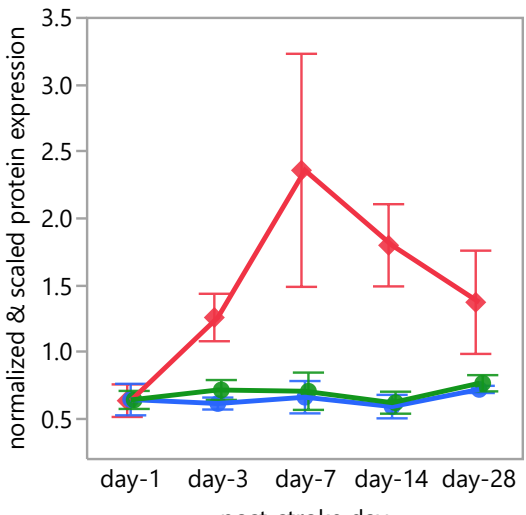

post-stroke day

\section{Unique_ID=Fermt3_Q8K1B8}

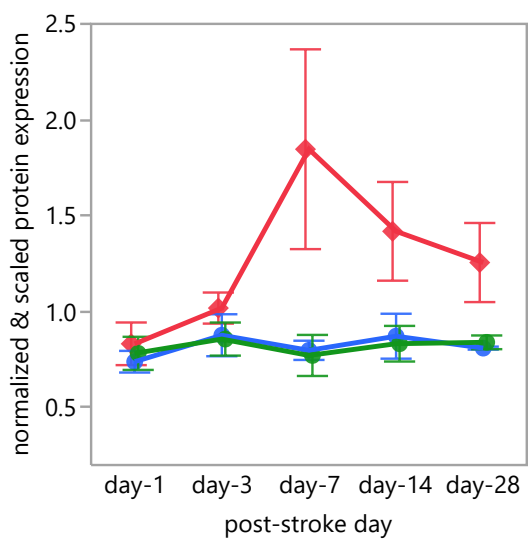

Unique_ID=Fgb_Q8K0E8

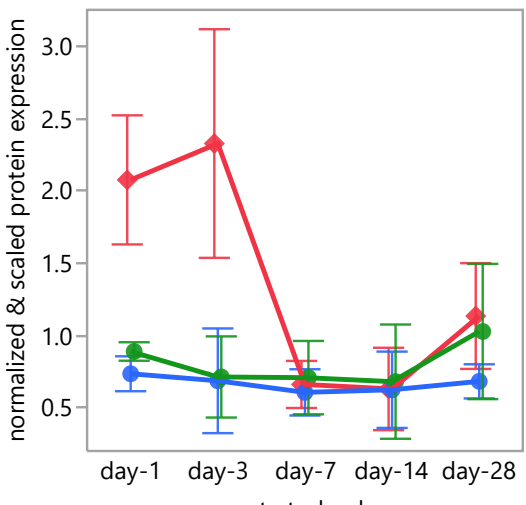

post-stroke day

\section{Unique_ID=Fhod1_Q6P9Q4}

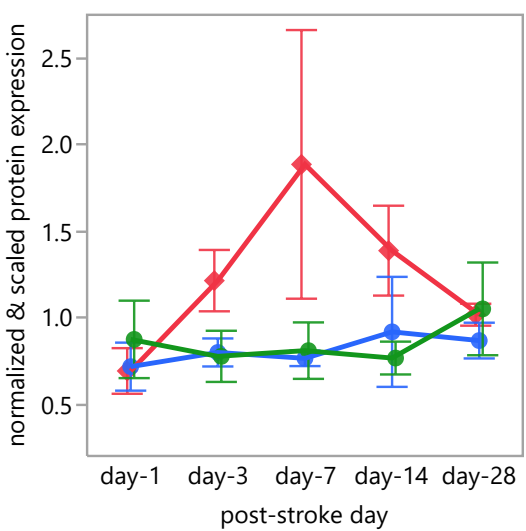

Unique_ID=Fcgr1_P26151

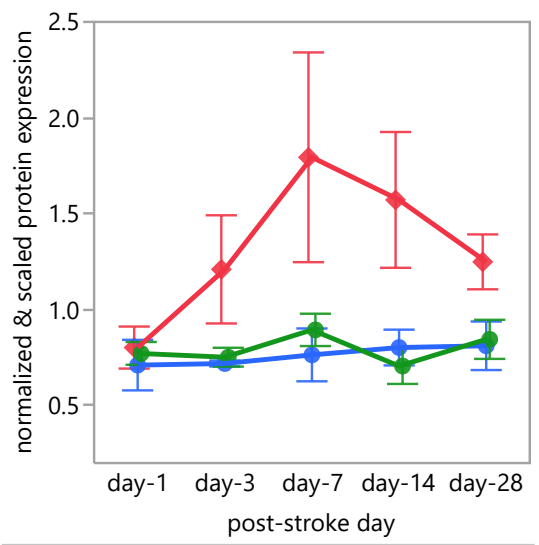

Unique_ID=Fetub_Q9QXC1

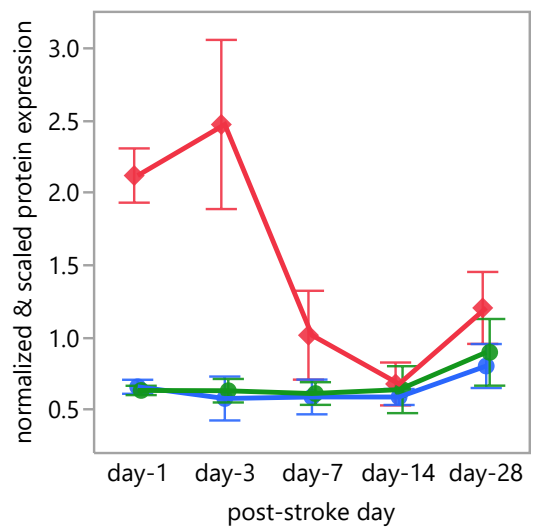

Unique_ID=Fgg_Q8VCM7

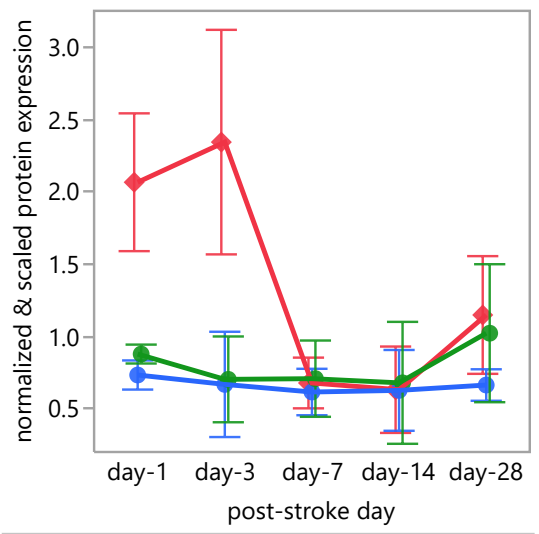

Unique_ID=Fkbp10_Q61576

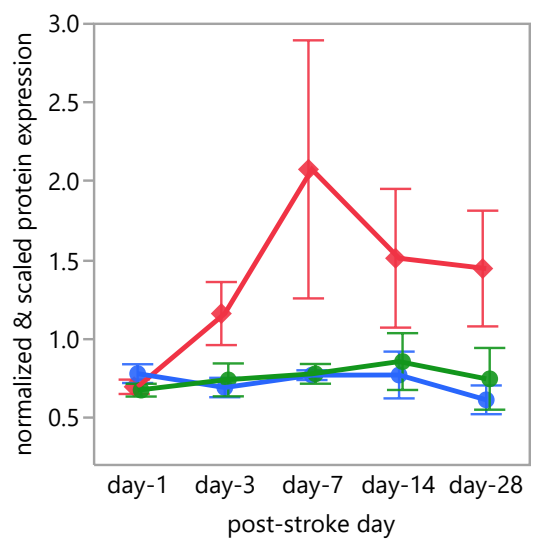




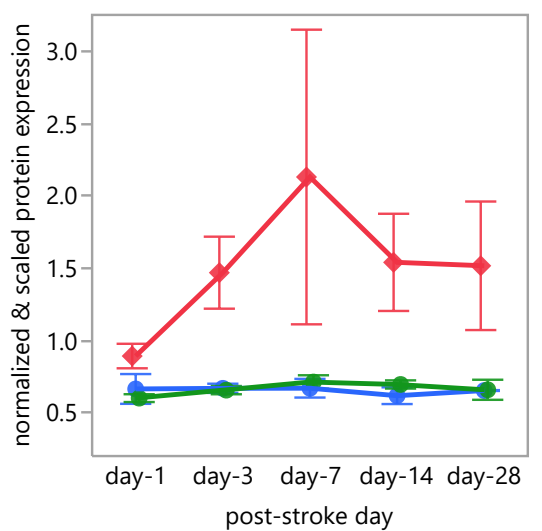

Unique_ID=Fstl1_Q62356

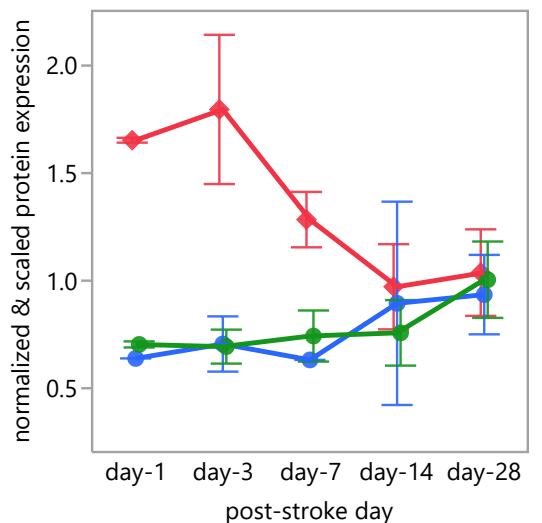

Unique_ID=Gbp2_Q9Z0E6

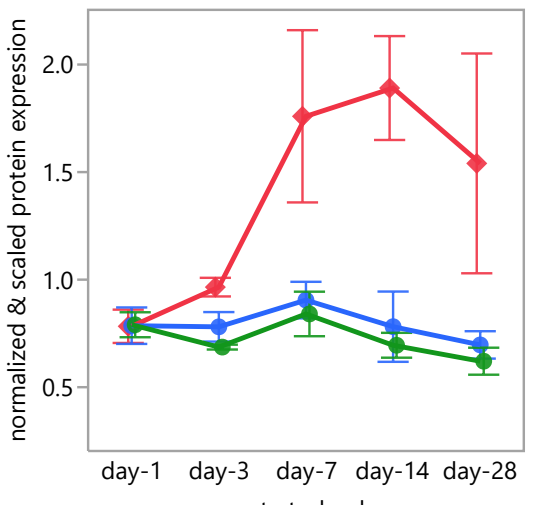

post-stroke day

Unique_ID=Gfap_P03995

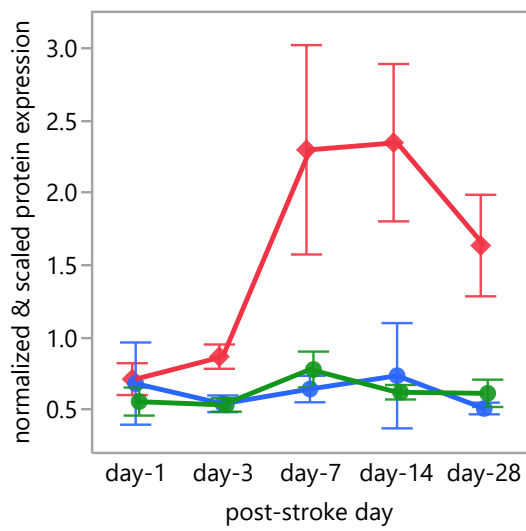

Figure_S2_309TopHits_Profile
Unique_ID=Flnc_Q8VHX6-2

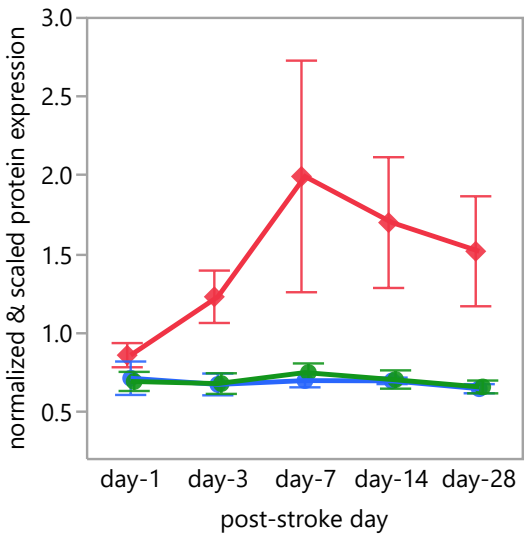

Unique_ID=Ftl1_P29391

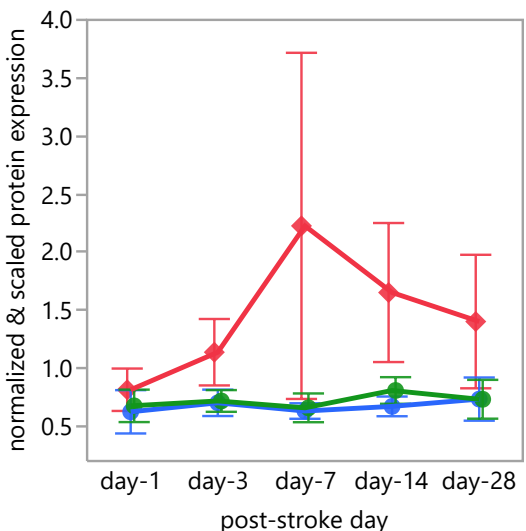

Unique_ID=Gbp4_Q61107

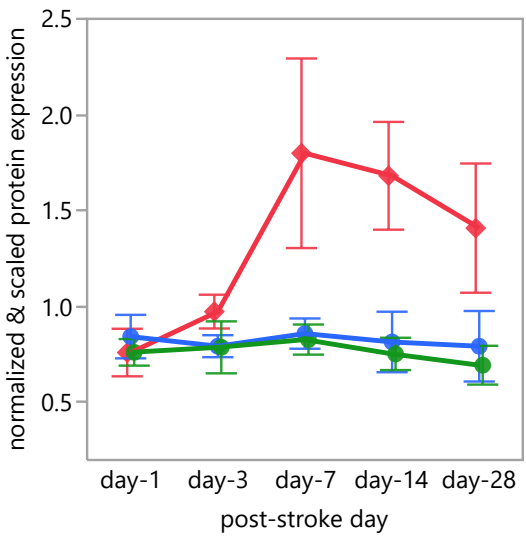

Unique_ID=Glipr2_Q9CYL5

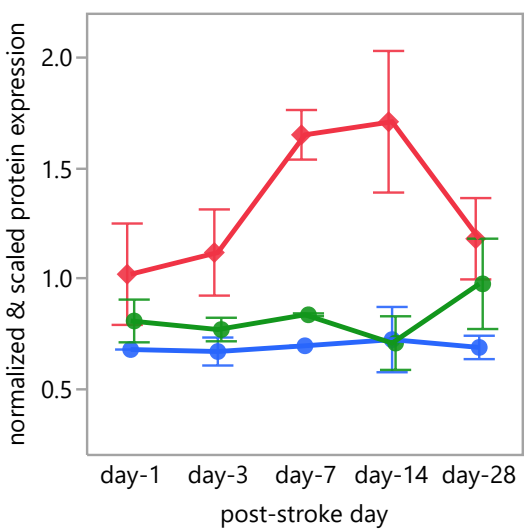

Unique_ID=Fn1_P11276

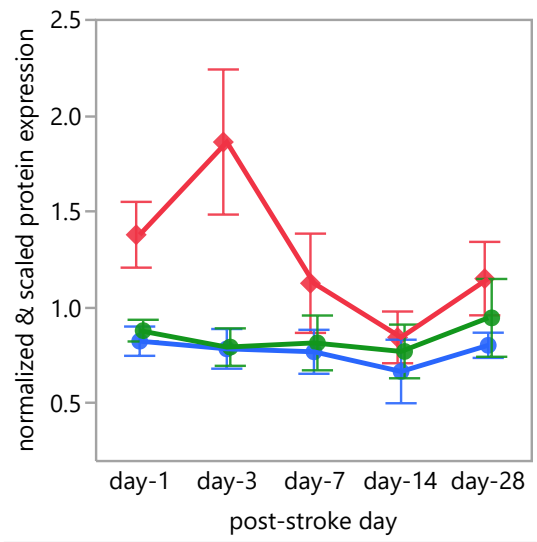

Unique_ID=Fyb_035601

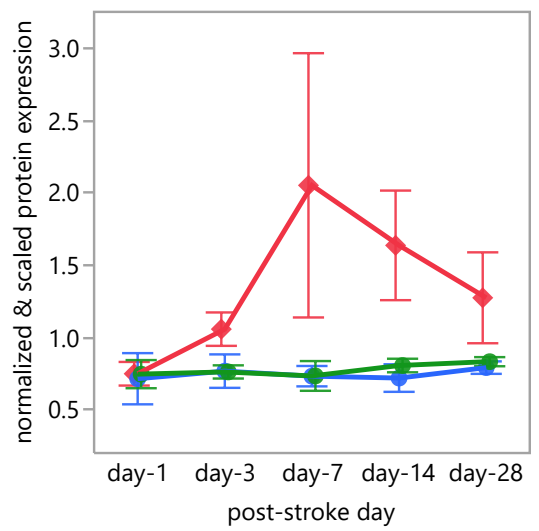

Unique_ID=Gc_P21614

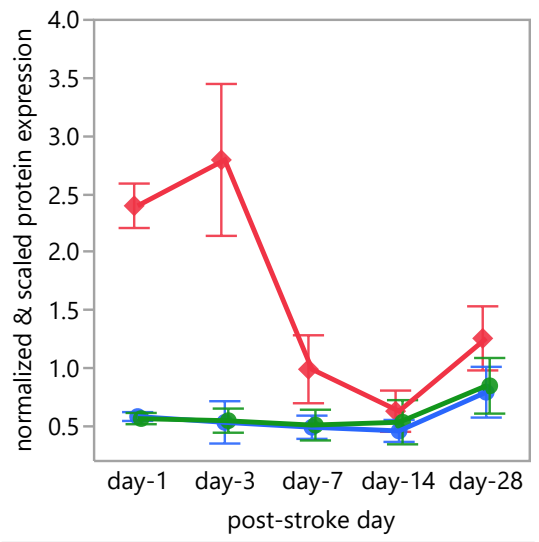

Unique_ID=Gpnmb_Q99P91

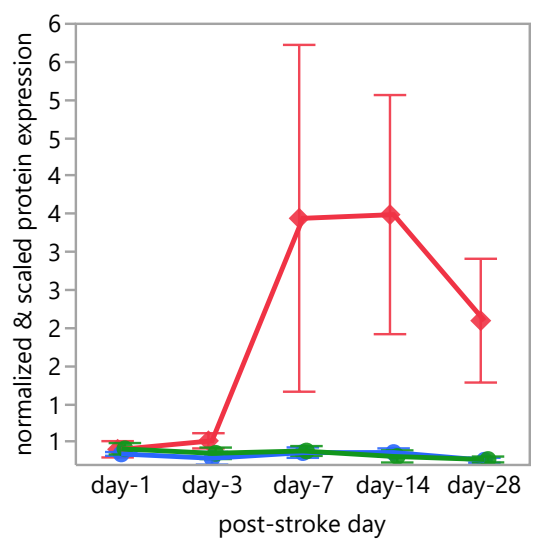




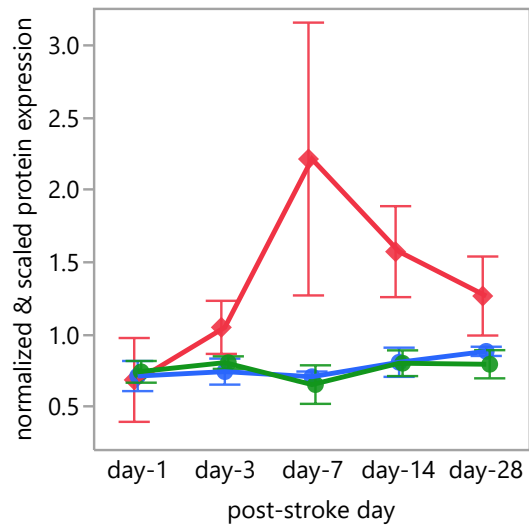

Unique_ID=Gsdmdc1_Q9D8T2

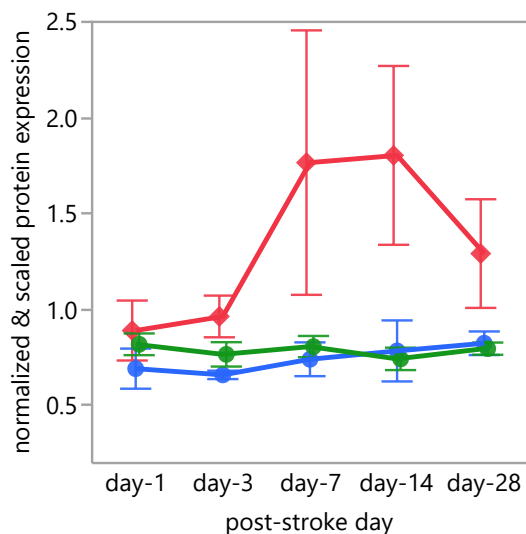

Unique_ID=H2-D1_P01899

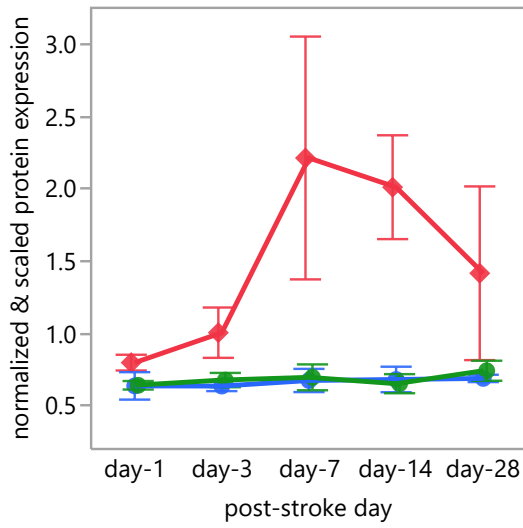

Unique_ID=Hcls1_P49710

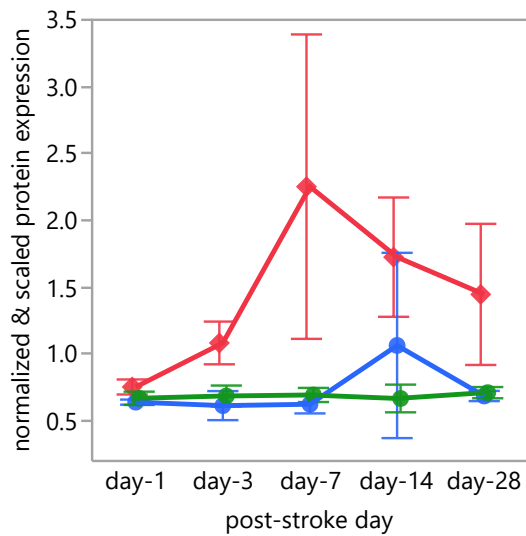

Figure_S2_309TopHits_Profile

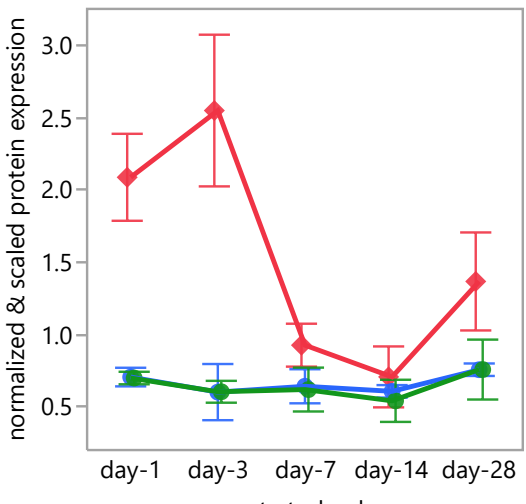

Unique_ID=Gsn_P13020-2

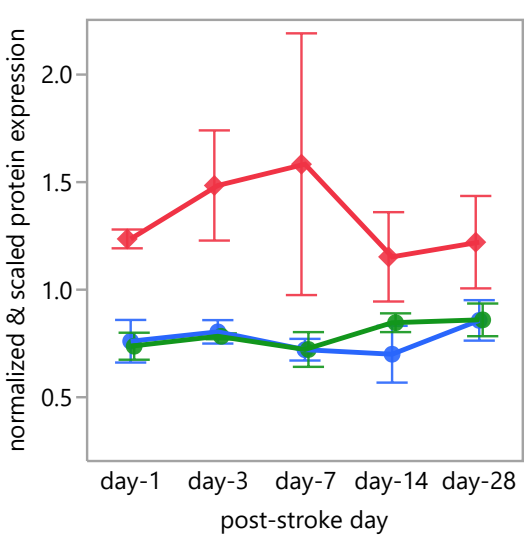

Unique_ID=H2-K1_P01901

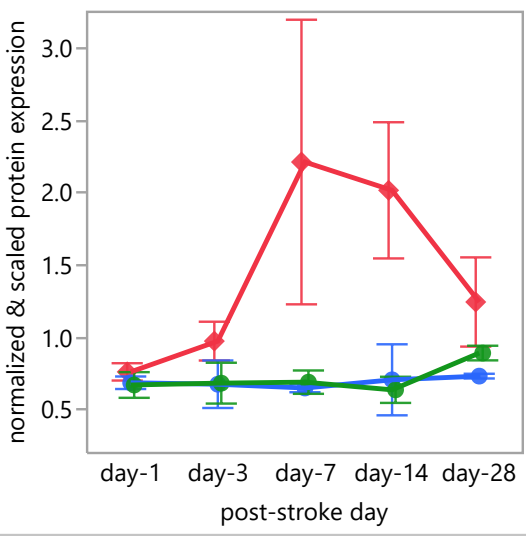

Unique_ID=Helz_Q6DFV5

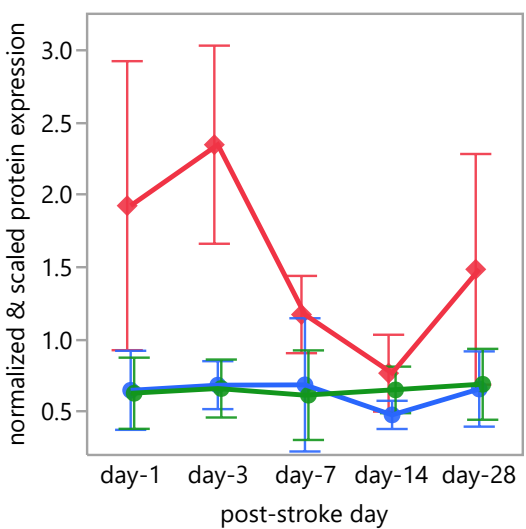

Unique_ID=Grn_P28798

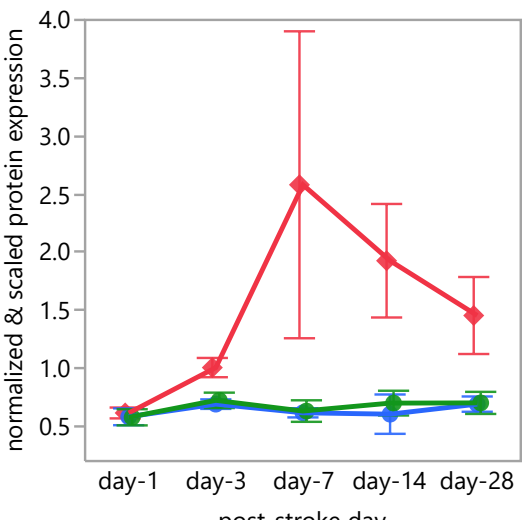

post-stroke day

Unique_ID=Gusb_P12265

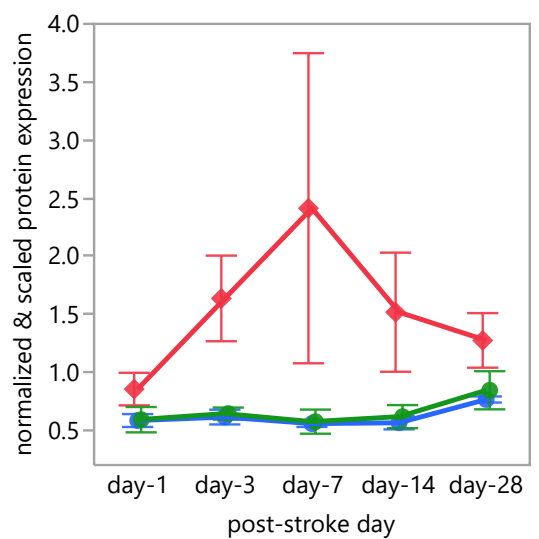

Unique_ID=H2-Q10_P01898

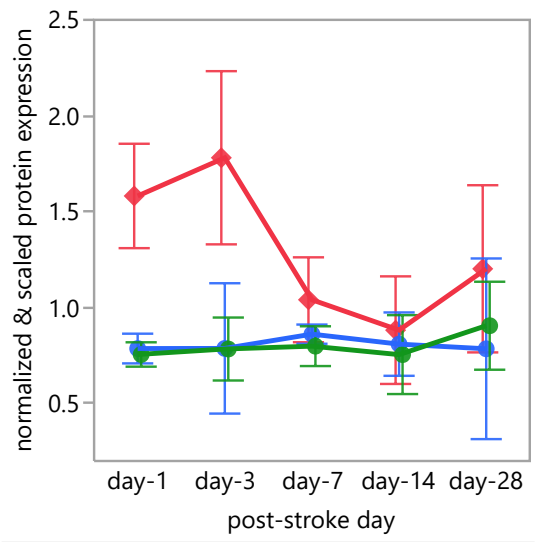

Unique_ID=Hexa_P29416

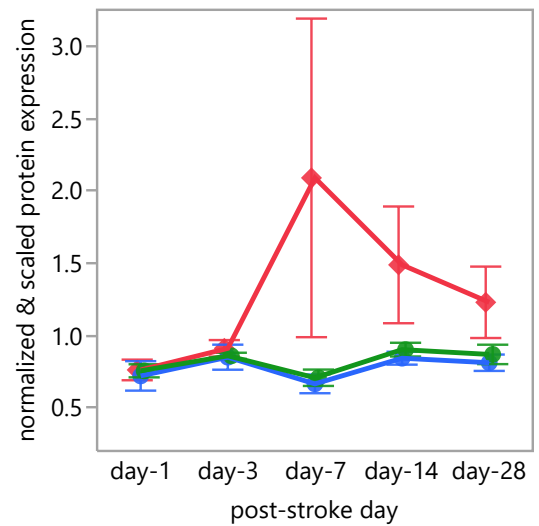




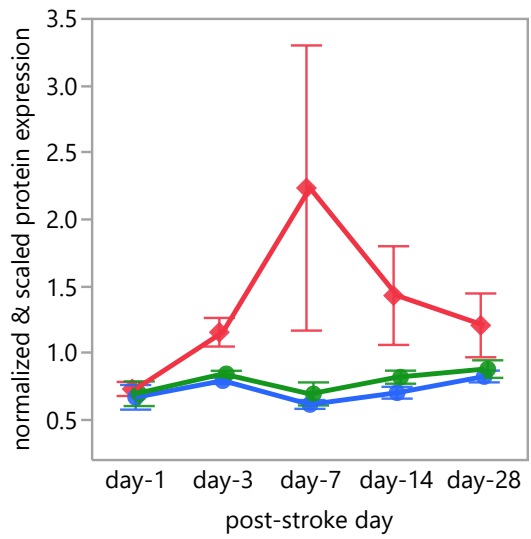

Unique_ID=Hmgcs2_P54869

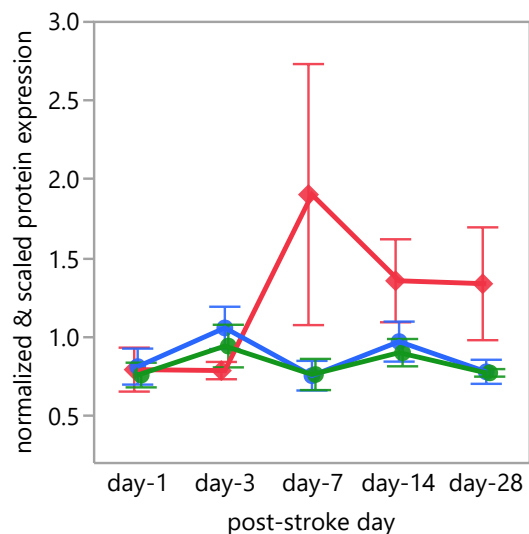

Unique_ID=Hpx_Q91X72

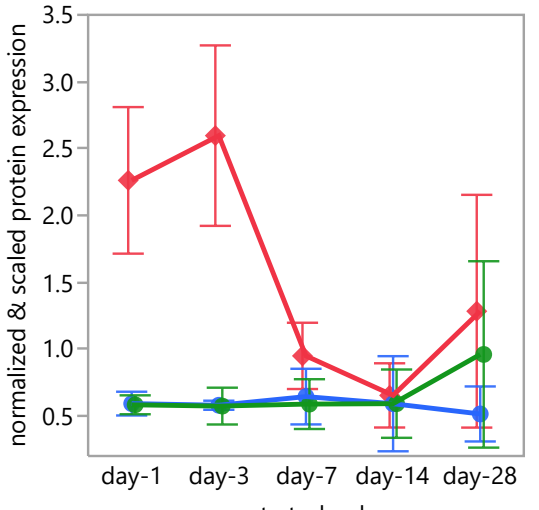

post-stroke day

\section{Unique_ID=Ifi204_P15092-2}

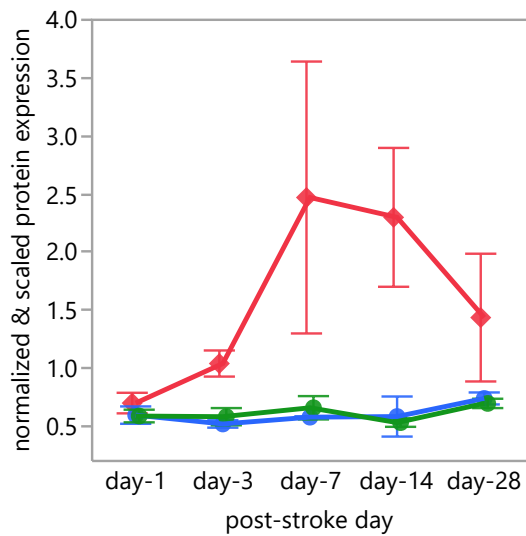

Figure_S2_309TopHits_Profile

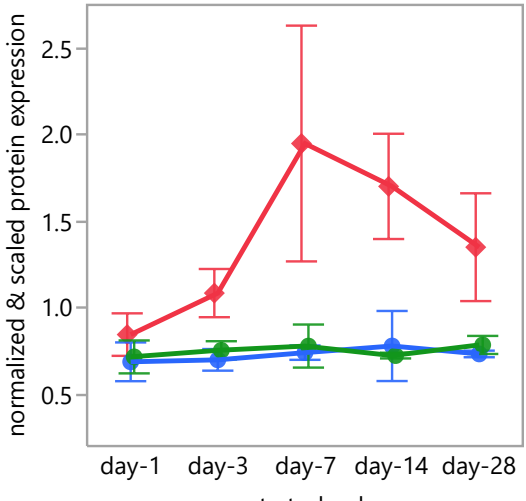

post-stroke day

Unique_ID=Hmha1_Q3TBD2

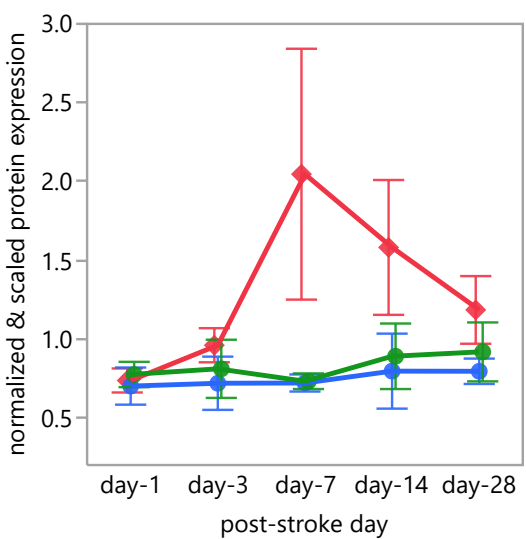

Unique_ID=Hrg_Q9ESB3

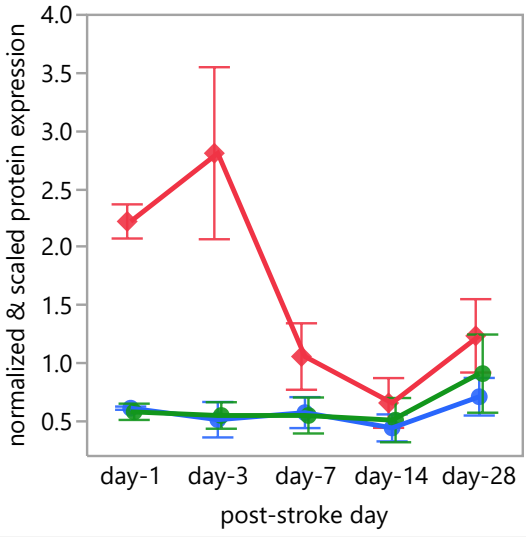

Unique_ID=Ifi30_Q9ESY9

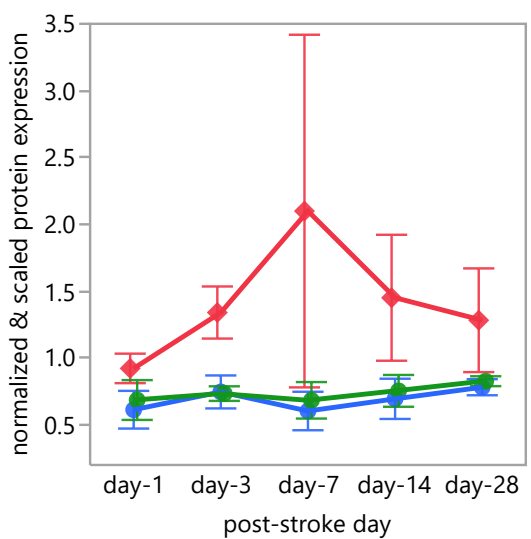

Unique_ID=Hk3_Q3TRM8

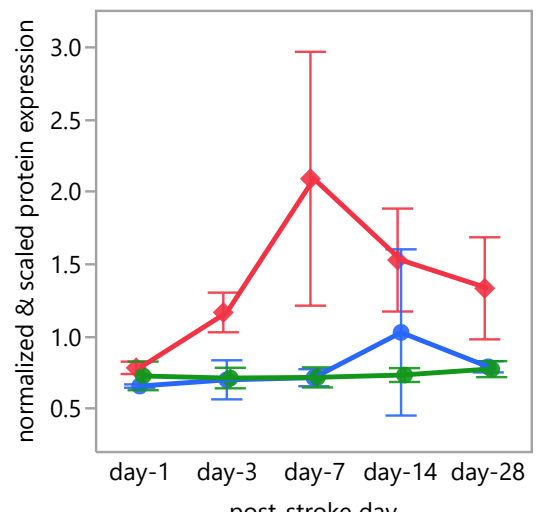

post-stroke day

Unique_ID=Hp_Q61646

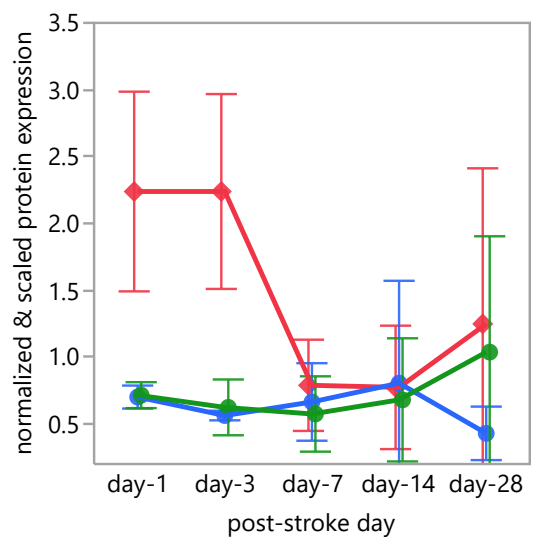

Unique_ID=Ica_Q9DBD0

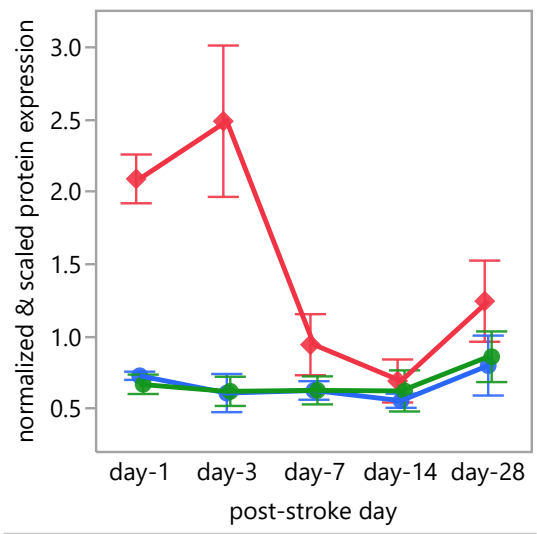

Unique_ID=Ifi35_Q9D8C4

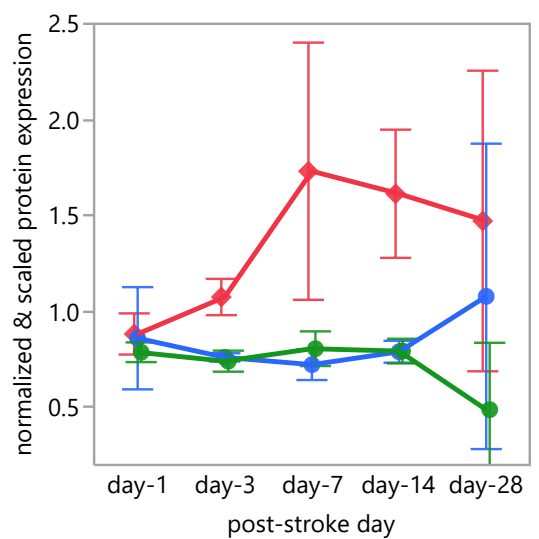




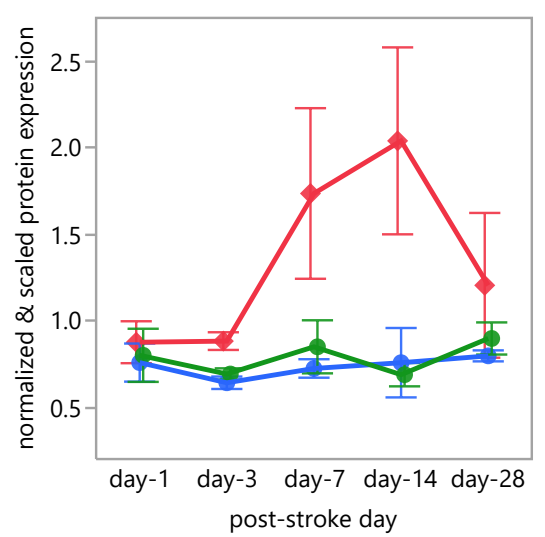

Unique_ID=Igfbp2_P47877

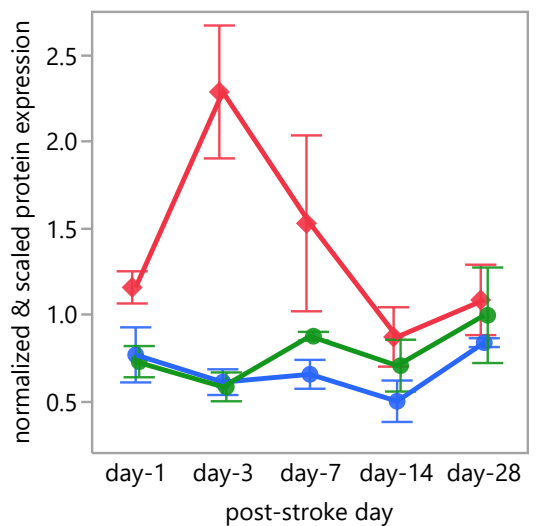

Unique_ID=lghg1_P01868

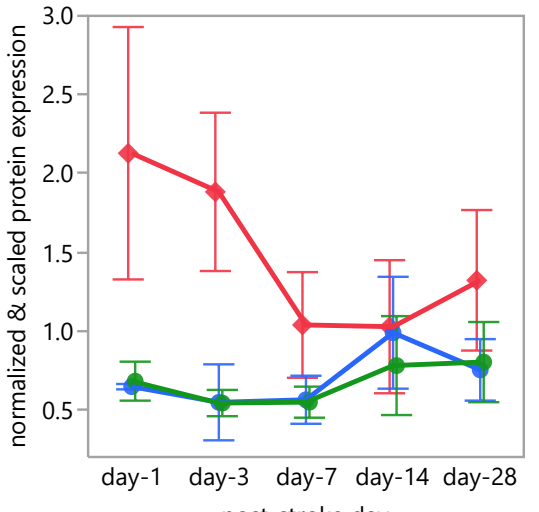

post-stroke day

Unique_ID=Inpp5d_Q9ES52-2

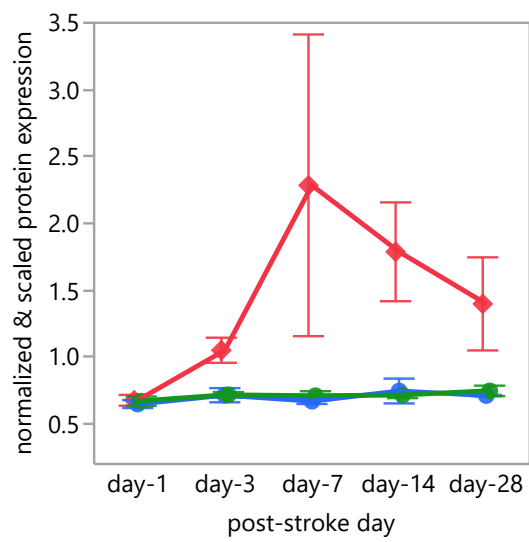

Figure_S2_309TopHits_Profile

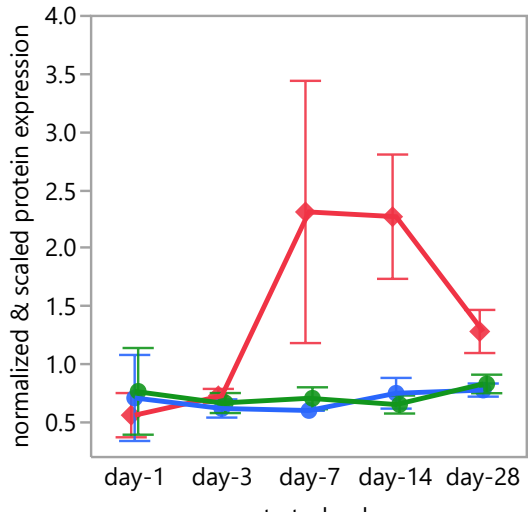

Unique_ID=Igfbp7_Q61581

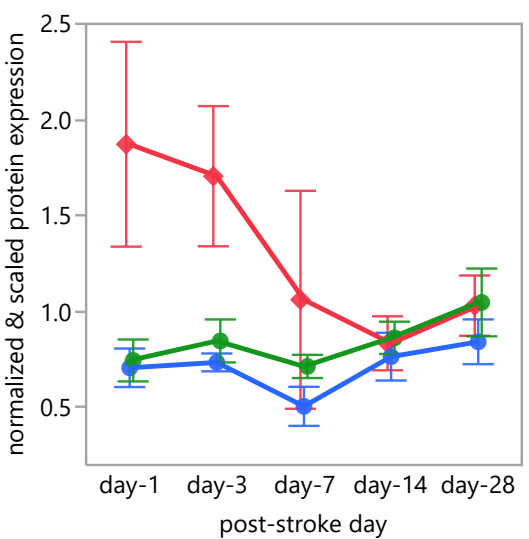

Unique_ID=Ighm_P01872

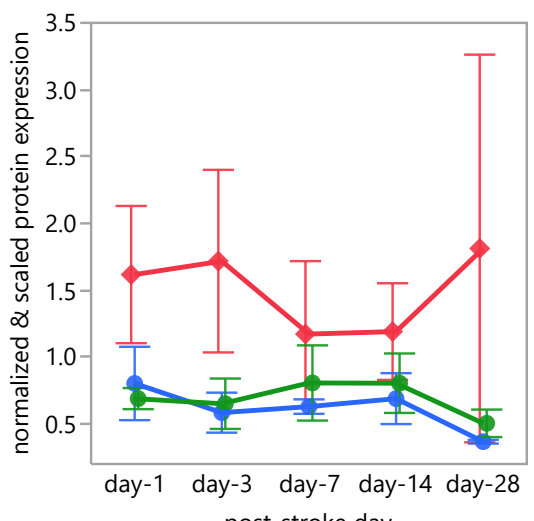

post-stroke day

\section{Unique_ID=Iqgap1_Q9JKF1}

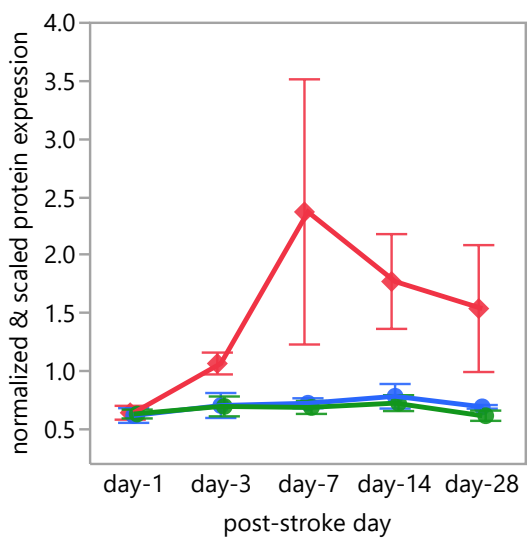

Unique_ID=Ifit3_Q64345

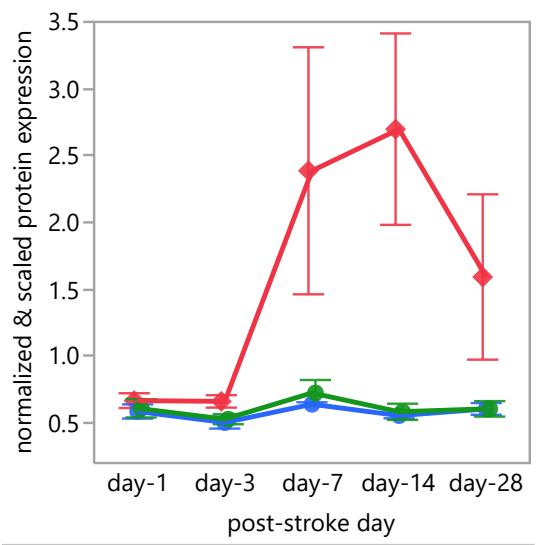

Unique_ID=Igh-3_P01867-2

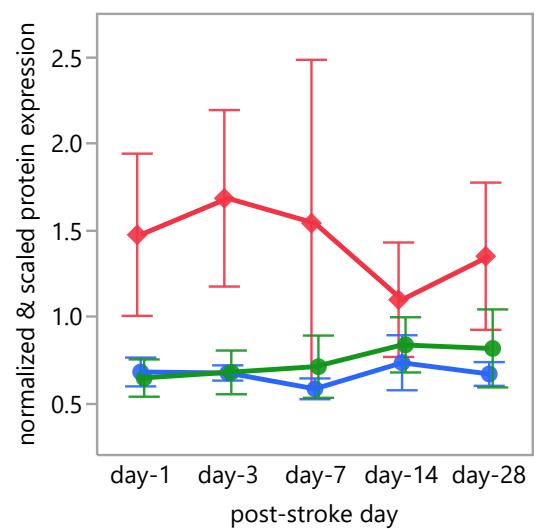

Unique_ID=II1rap_Q61730

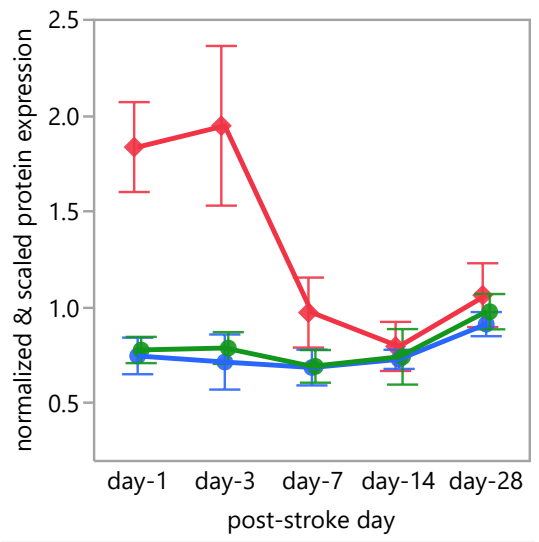

Unique_ID=Isg15_Q64339

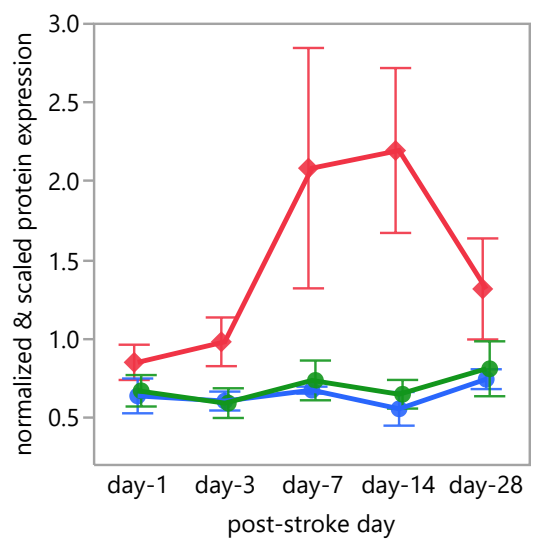




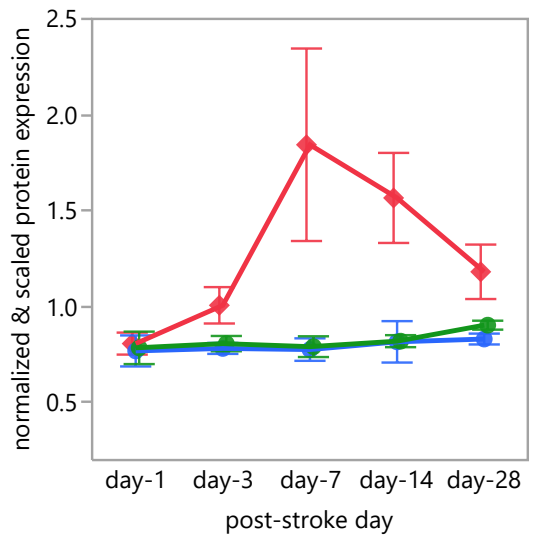

Unique_ID=Itih1_Q61702

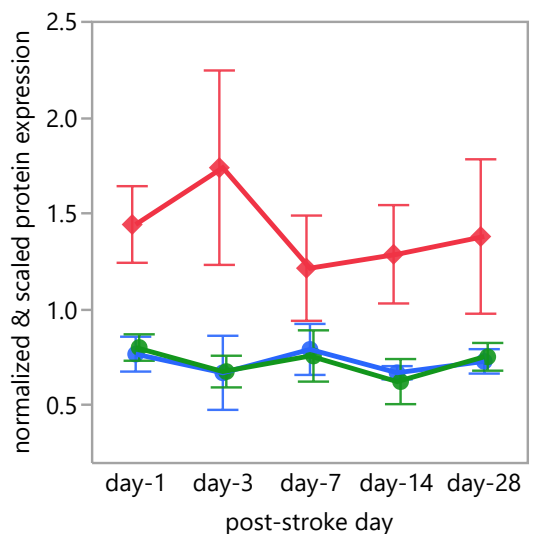

Unique_ID=Kif3c_035066

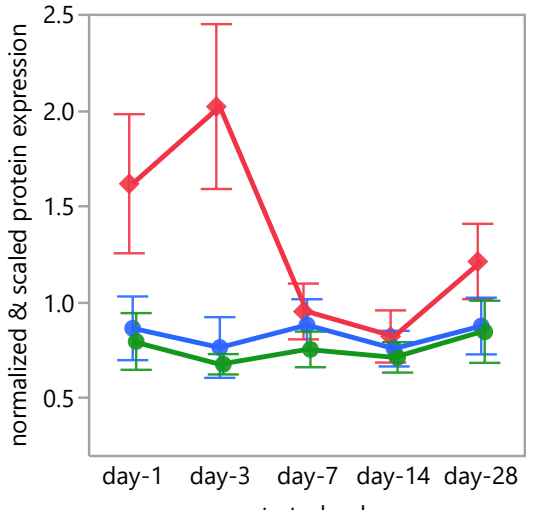

post-stroke day

Unique_ID=Lag3_Q61790

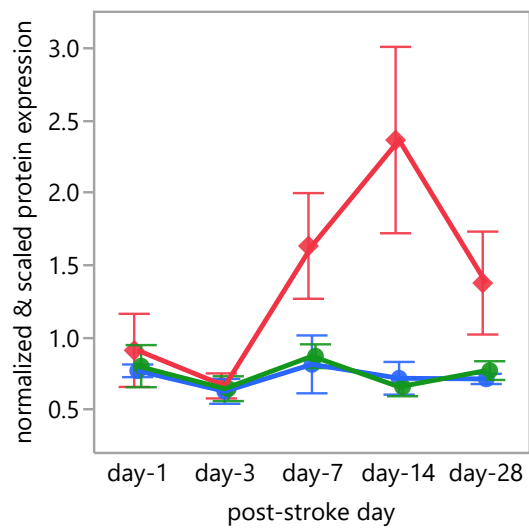

Figure_S2_309TopHits_Profile

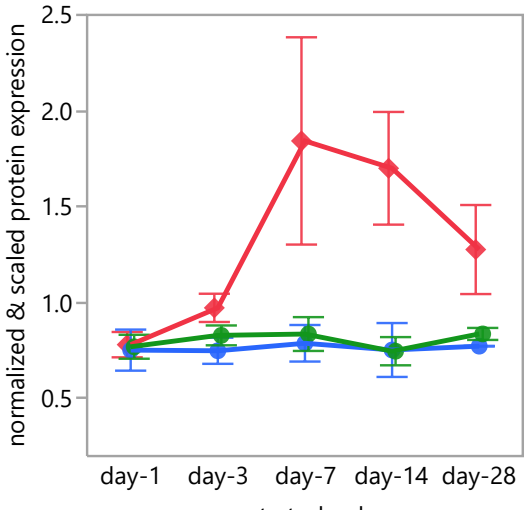

post-stroke day

Unique_ID=Itih2_Q61703

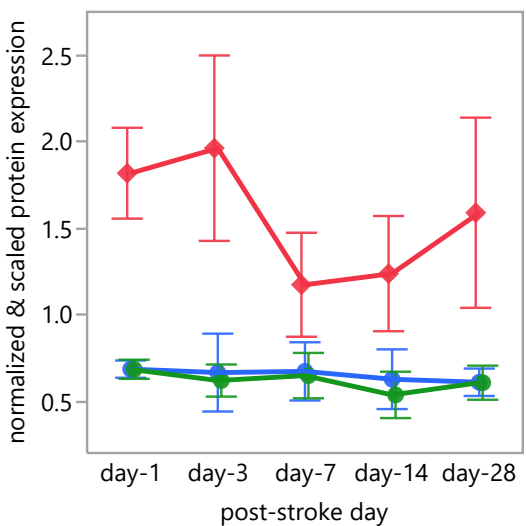

Unique_ID=KIkb1_P26262

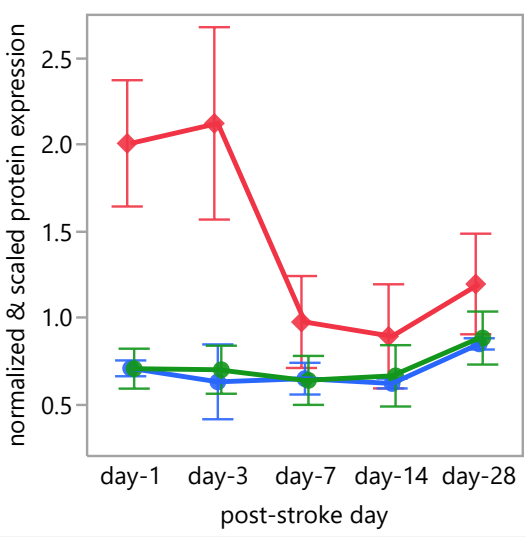

Unique_ID=Lamp1_P11438

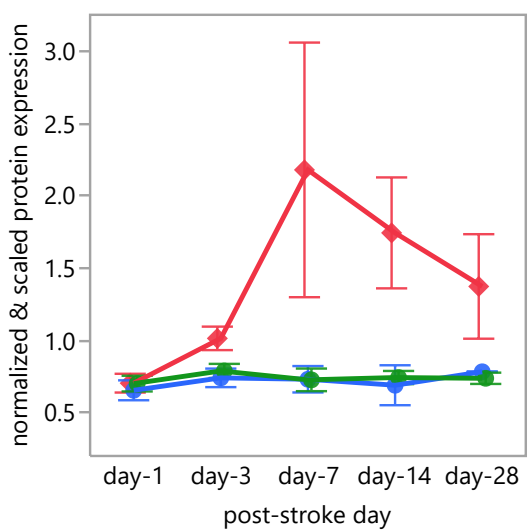

Unique_ID=Itgb5_070309

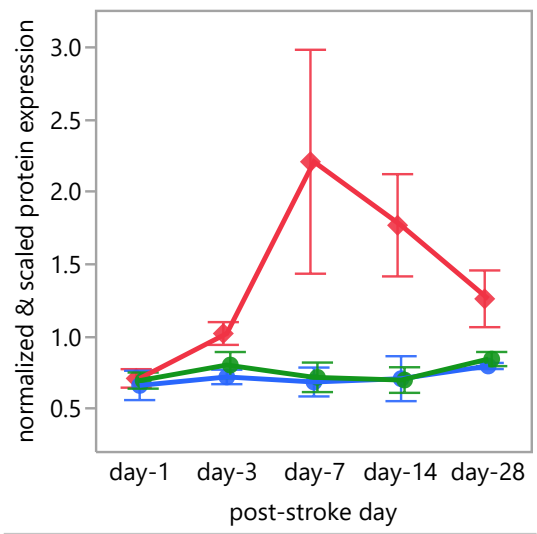

Unique_ID=Itih4_A6X935

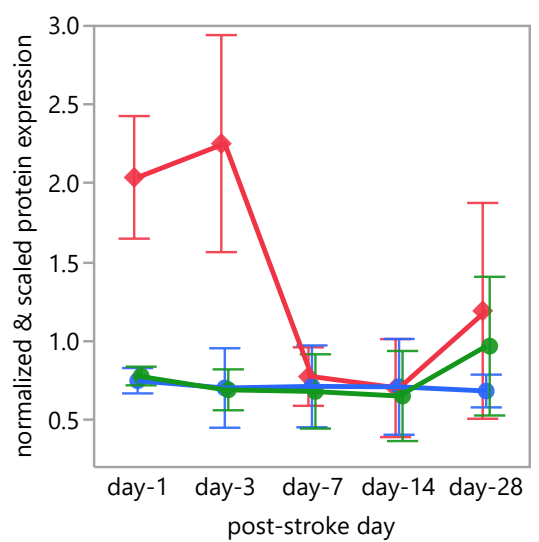

Unique_ID=Kng1_008677-2

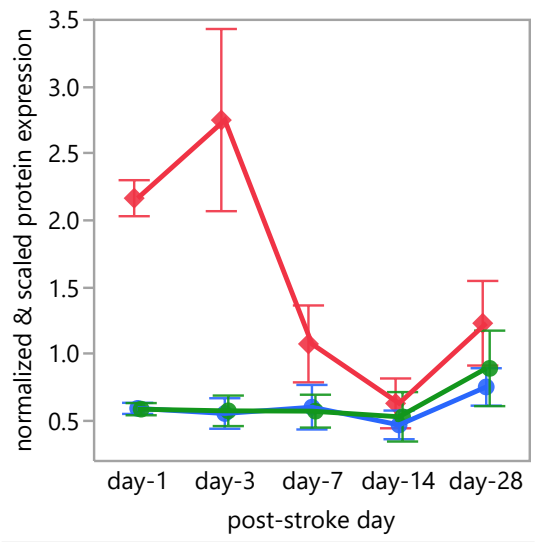

Unique_ID=Lamp2_P17047-3

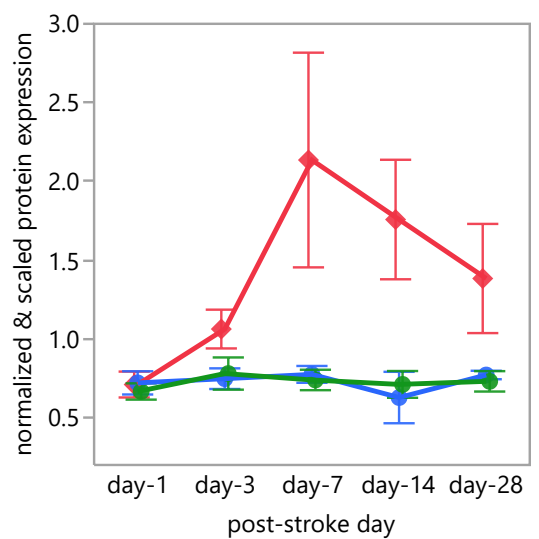




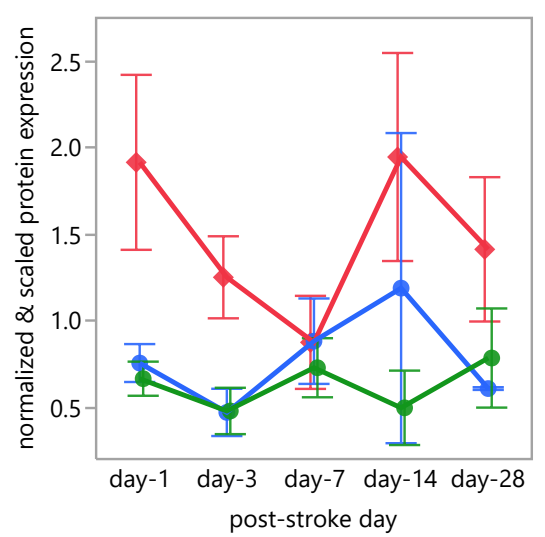

Unique_ID=Lgals3_P16110

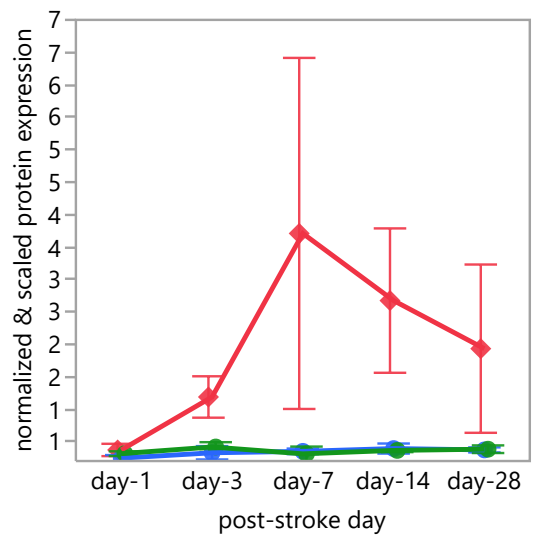

Unique_ID=Lgmn_O89017

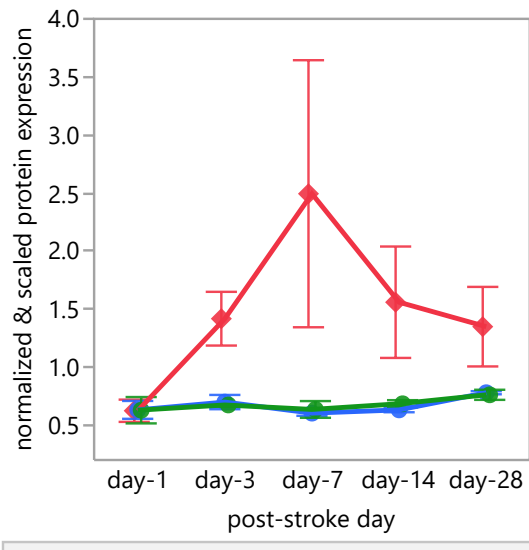

Unique_ID=Lpxn_Q99N69

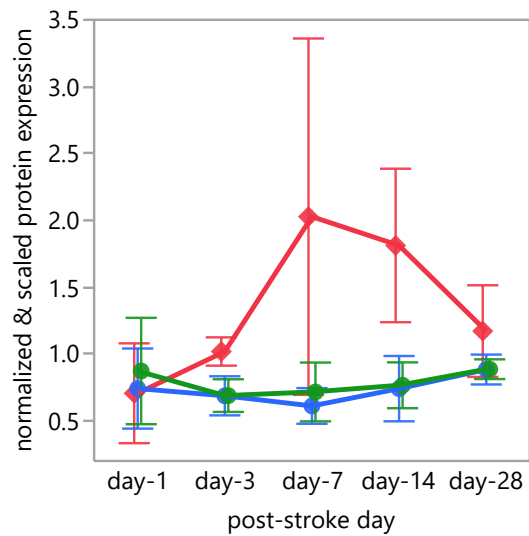

Figure_S2_309TopHits_Profile

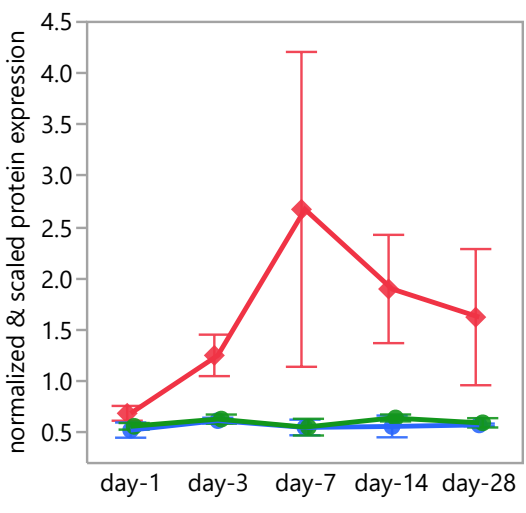

post-stroke day

\section{Unique_ID=Lgals3bp_Q07797}

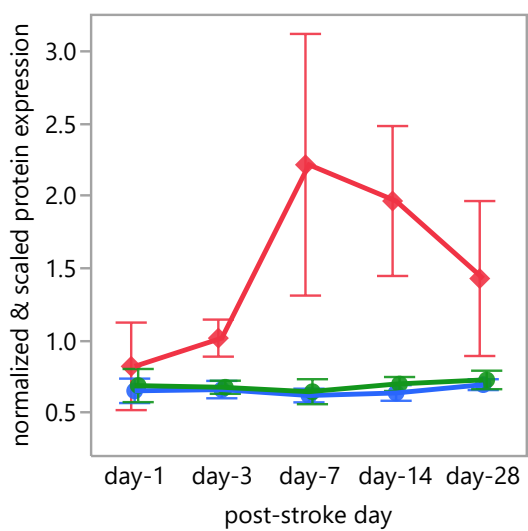

Unique_ID=Lifr_P42703-2

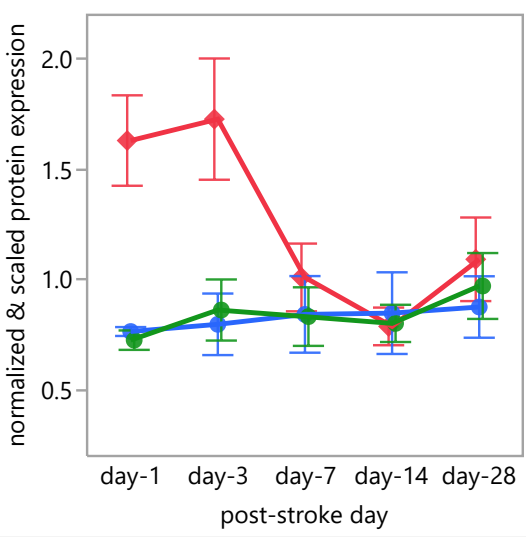

Unique_ID=Lrrfip1_Q3UZ39

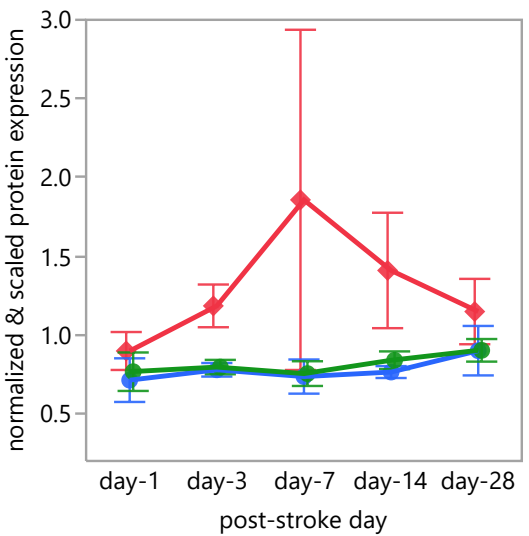

Unique_ID=Lgals1_P16045

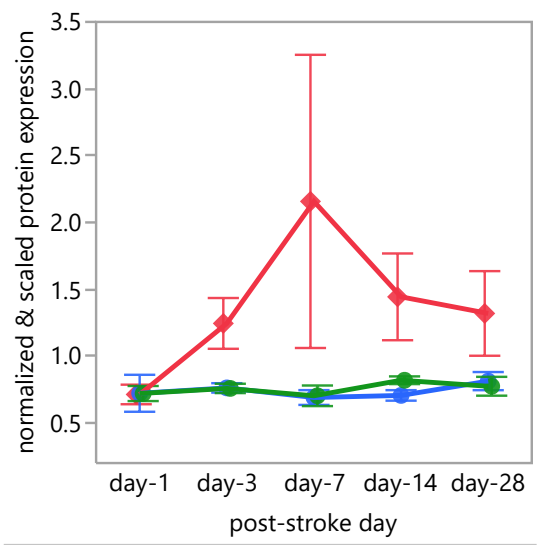

Unique_ID=Lgals9_008573-3

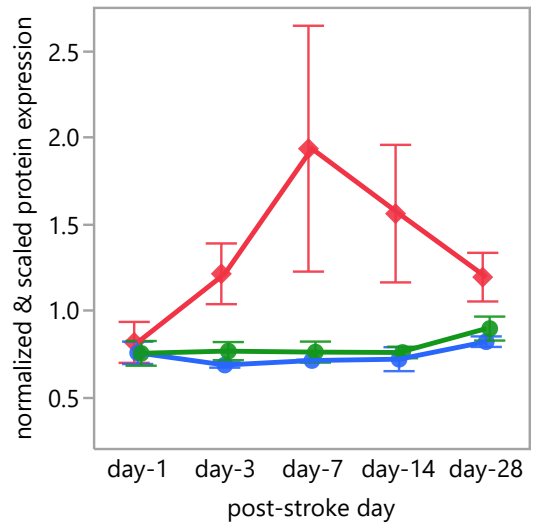

Unique_ID=Lipa_Q9Z0M5

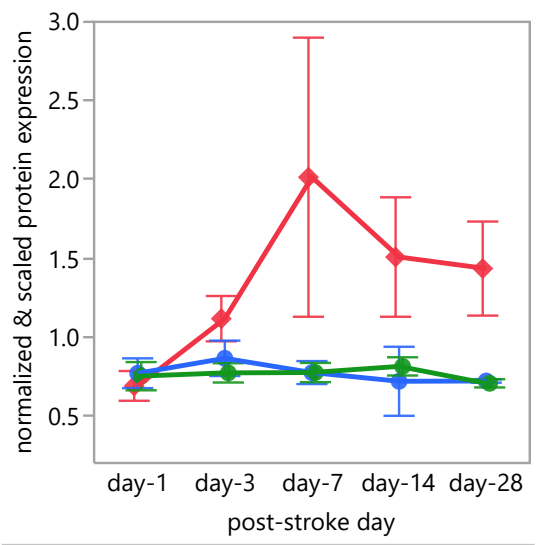

Unique_ID=Lsp1_P19973-2

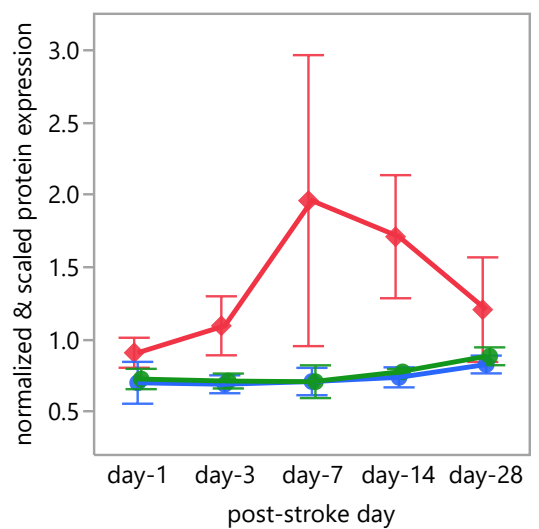




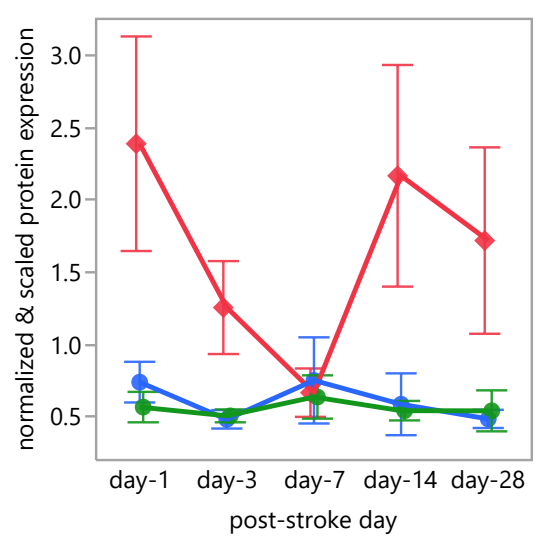

Unique_ID=Lyz2_P08905

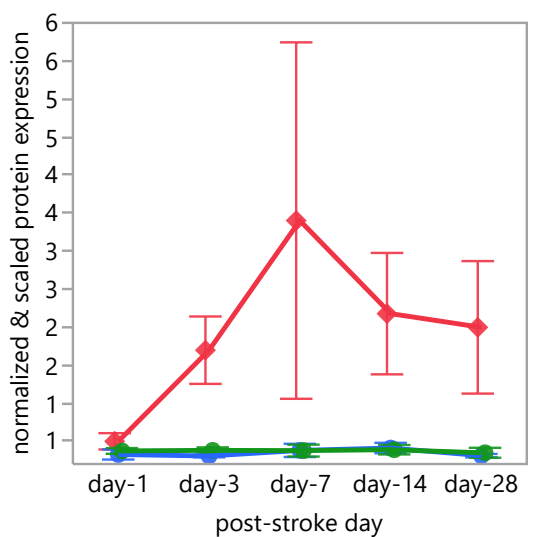

Unique_ID=Mcm2_P97310

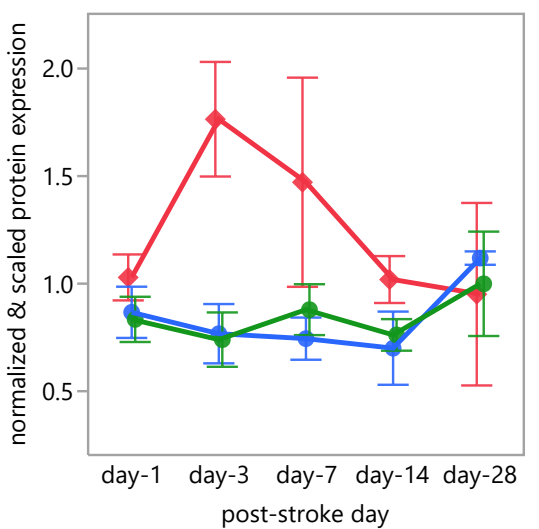

Unique_ID=Mcm6_P97311

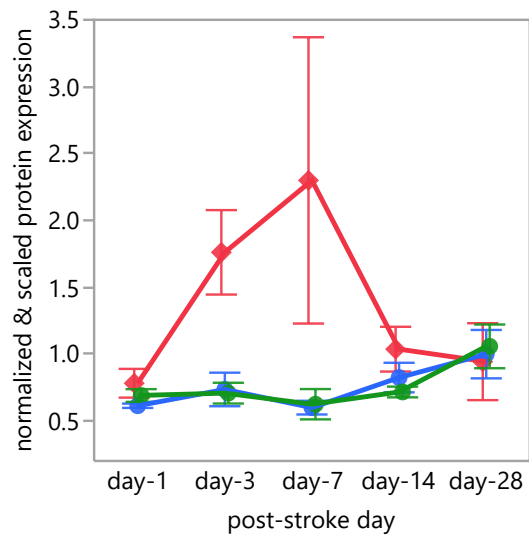

Figure_S2_309TopHits_Profile

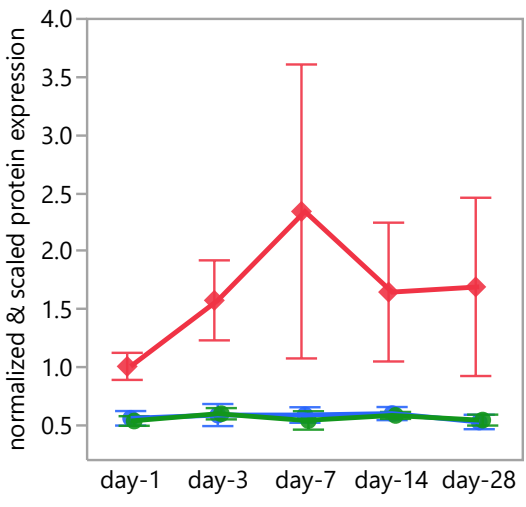

post-stroke day

Unique_ID=Mag_P20917

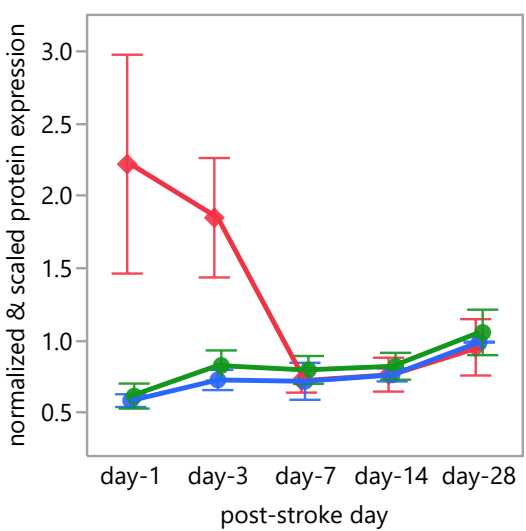

Unique_ID=Mcm3_P25206

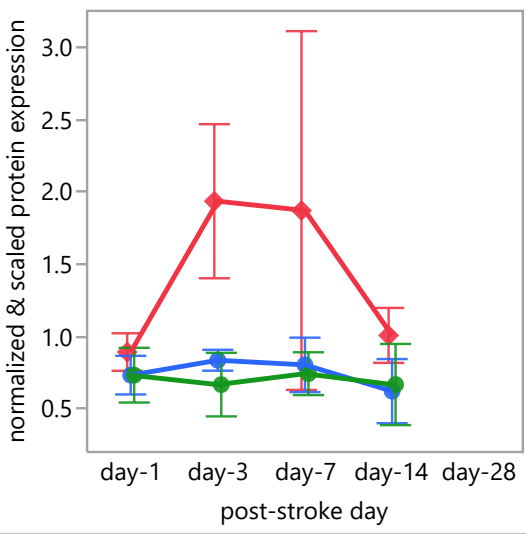

Unique_ID=Mrc2_Q64449

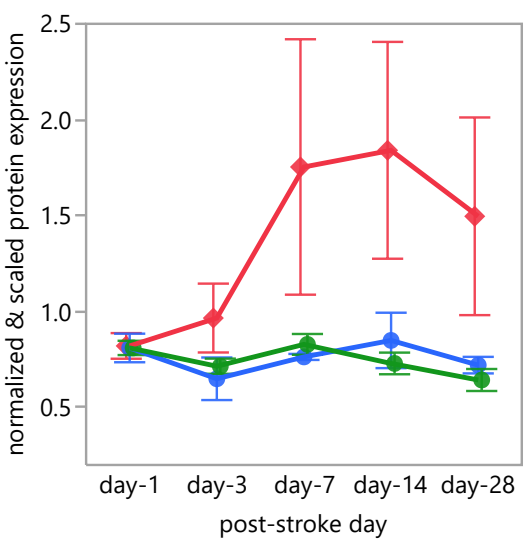

Unique_ID=Ly86_088188

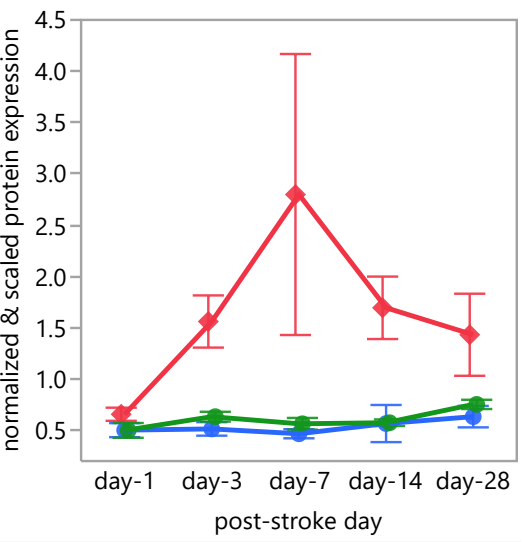

Unique_ID=Man2b1_009159

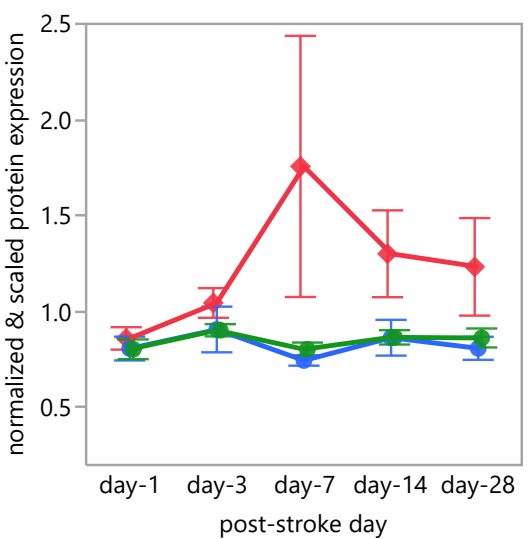

Unique_ID=Mcm4_P49717

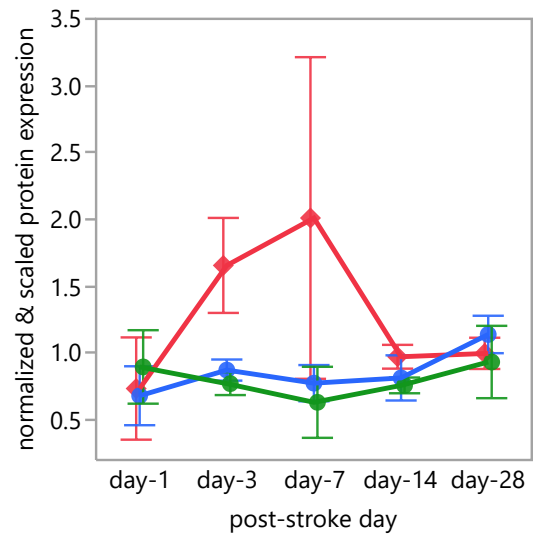

Unique_ID=Msn_P26041

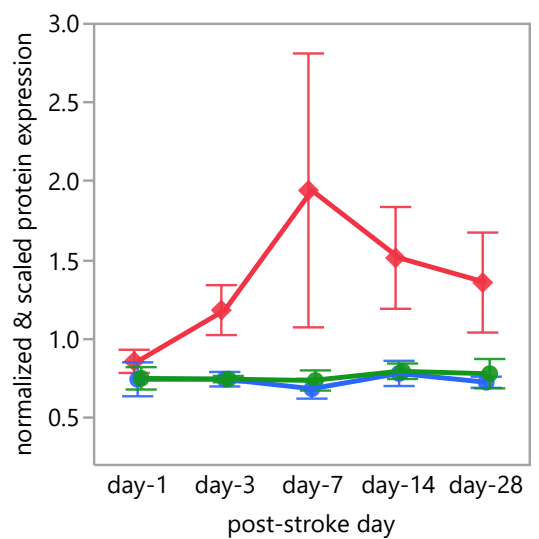




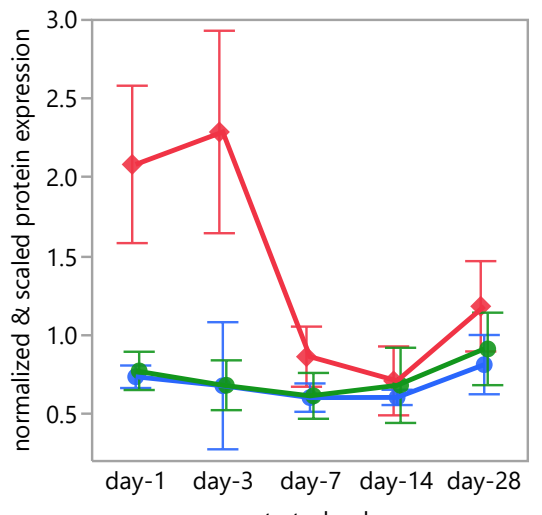

\section{Unique_ID=Mvp_Q9EQK5}

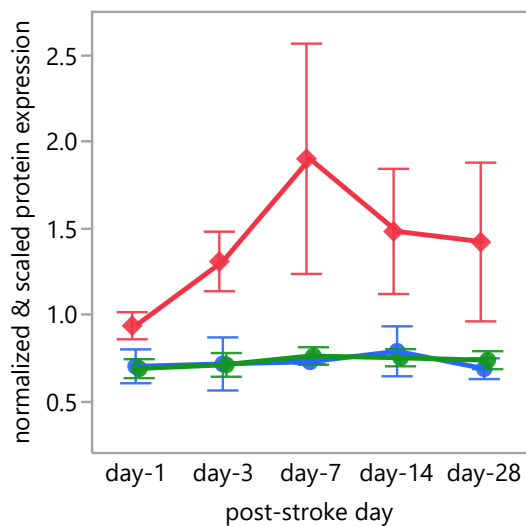

Unique_ID=Myo1f_P70248

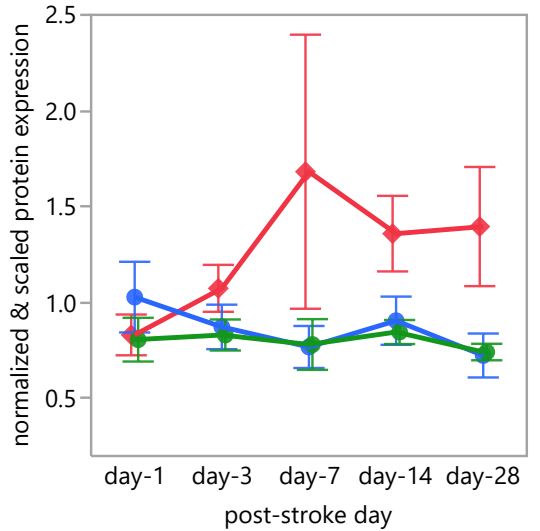

Unique_ID=Ncf2_070145

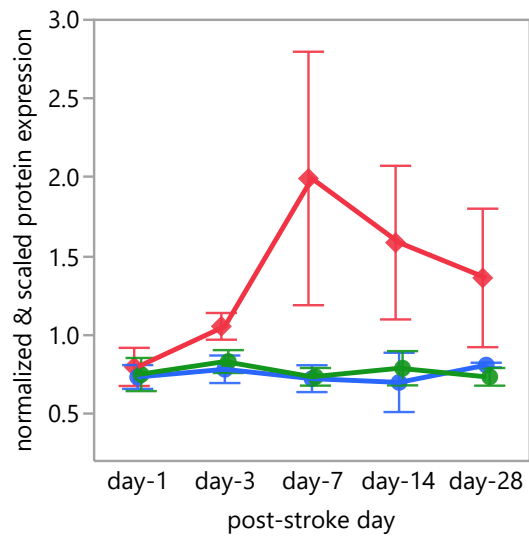

Figure_S2_309TopHits_Profile

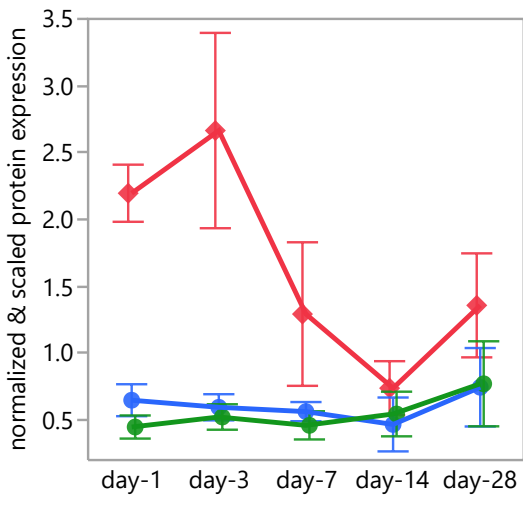

post-stroke day

\section{Unique_ID=Myh9_Q8VDD5}

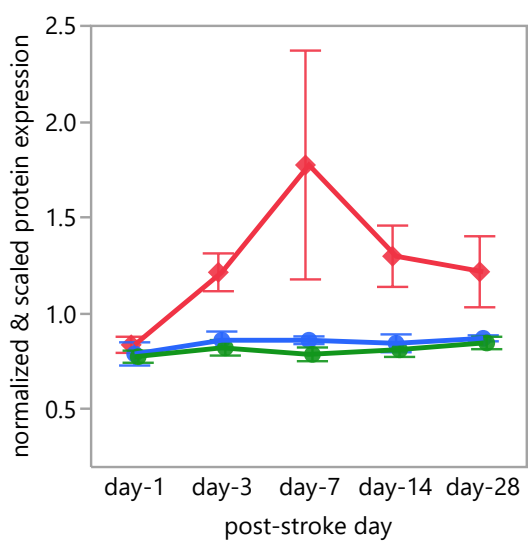

Unique_ID=Myof_Q69ZN7-4

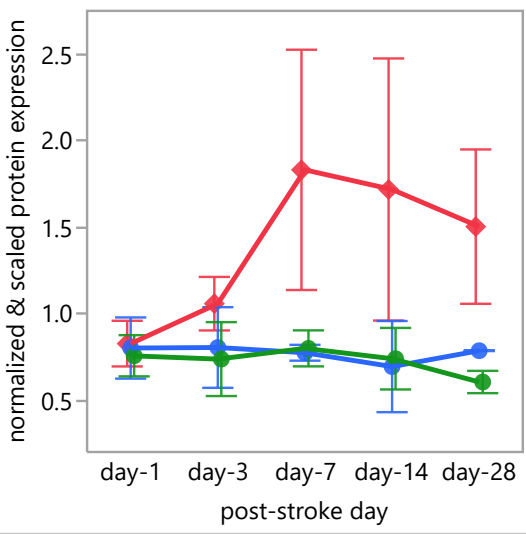

Unique_ID=Nes_Q6P5H2-2

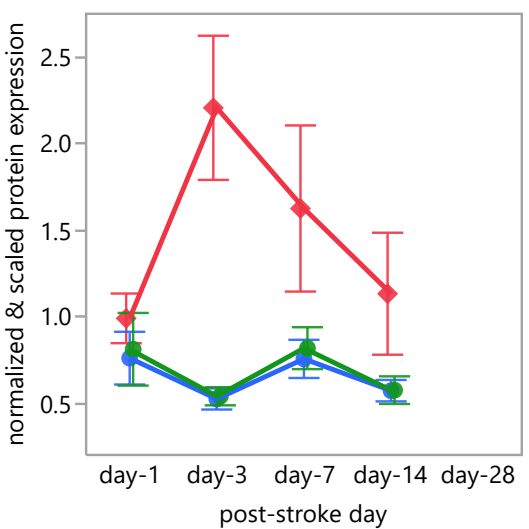

Unique_ID=Mup4_P11590

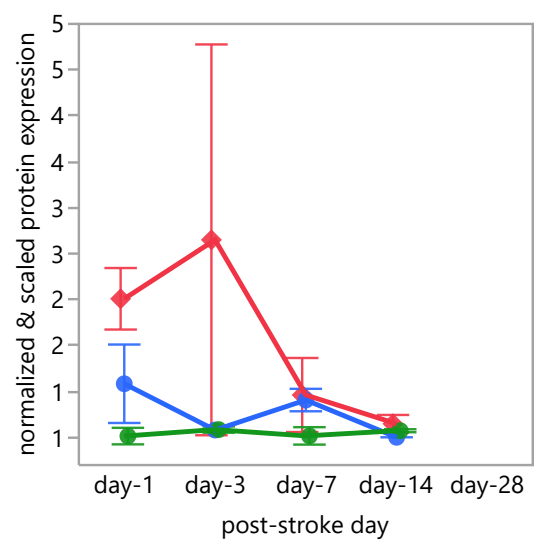

Unique_ID=Myo1e_E9Q634

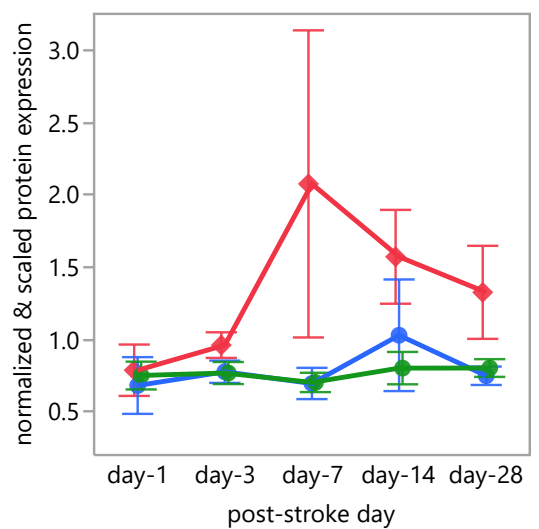

Unique_ID=Ncf1_Q09014

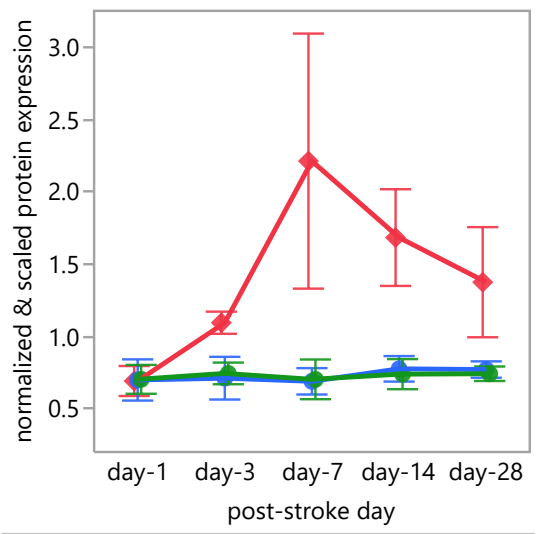

Unique_ID=Nfkb2_Q9WTK5

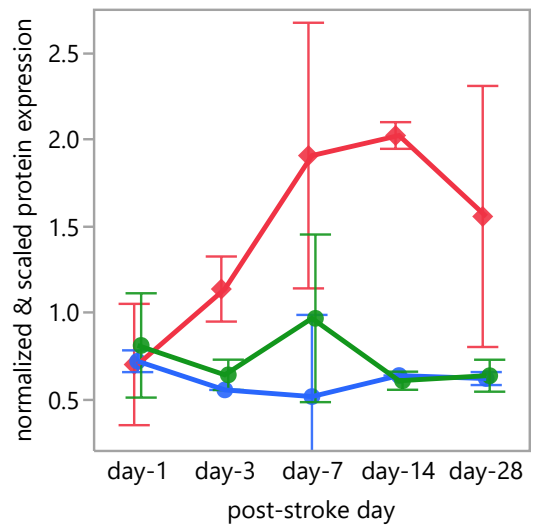




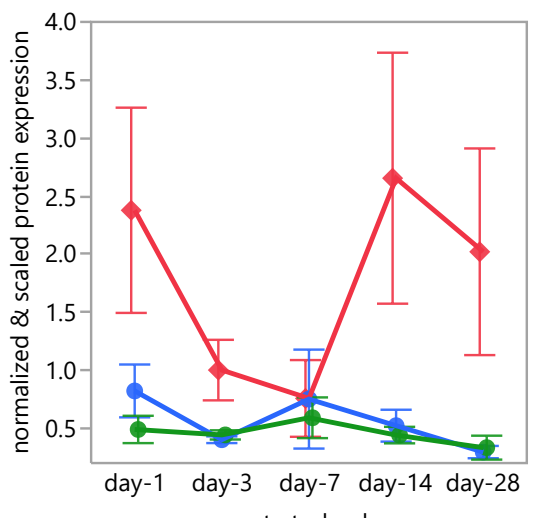

post-stroke day

\section{Unique_ID=Olfml3_Q8BK62}

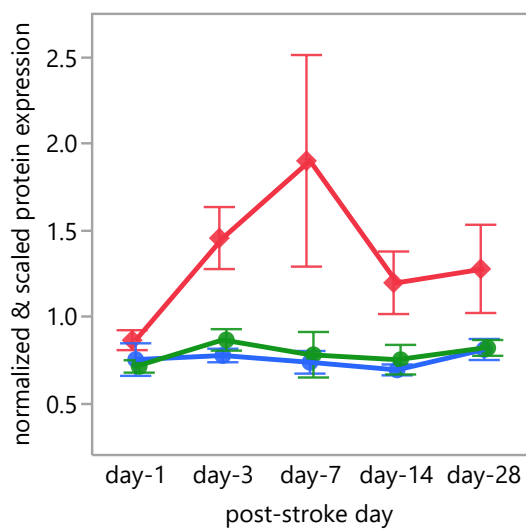

Unique_ID=P01631_P01631

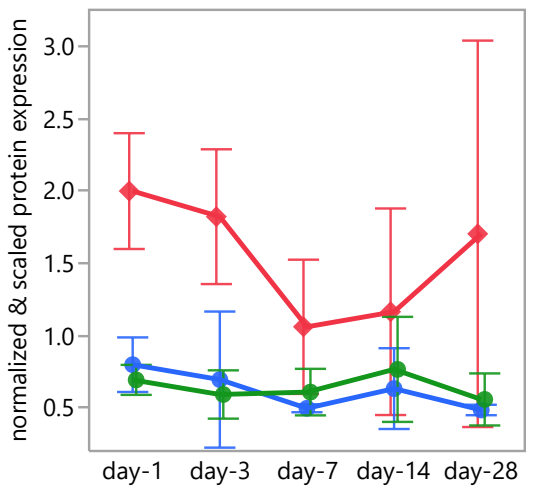

post-stroke day

\section{Unique_ID=P01837_P01837}

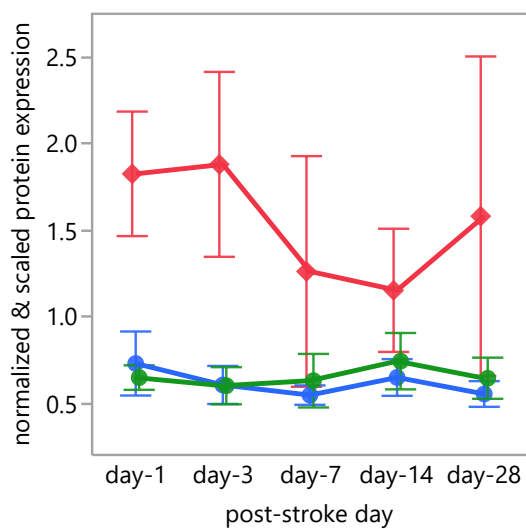

Figure_S2_309TopHits_Profile

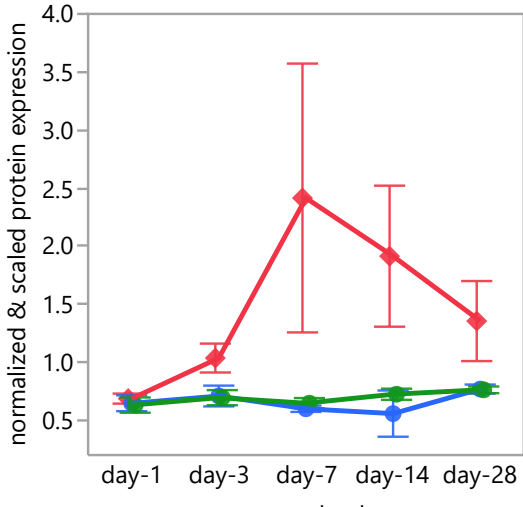

post-stroke day

Unique_ID=Orm1_Q60590

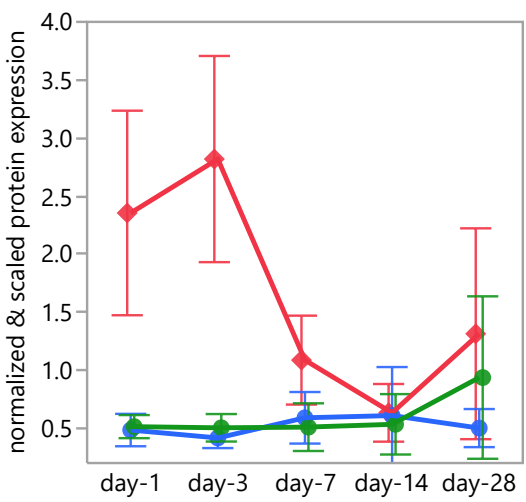

post-stroke day

Unique_ID=P01646_P01646

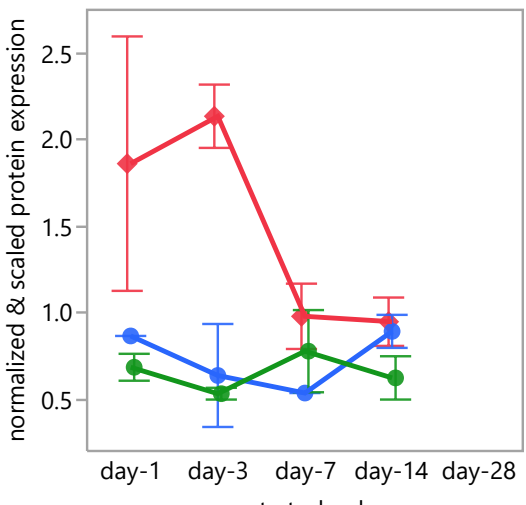

Unique_ID=P01864 P01864

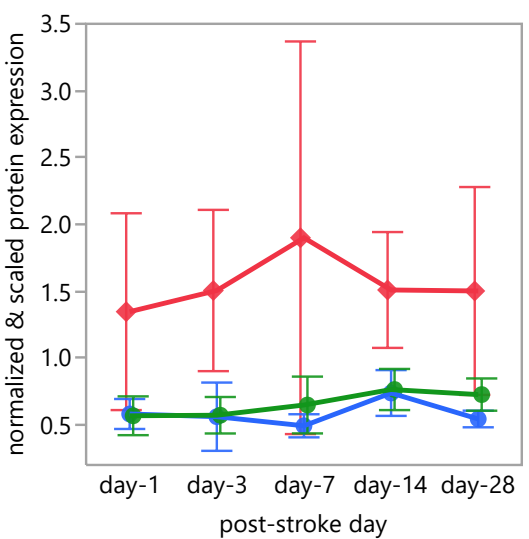

Unique_ID=NpI_Q9DCJ9

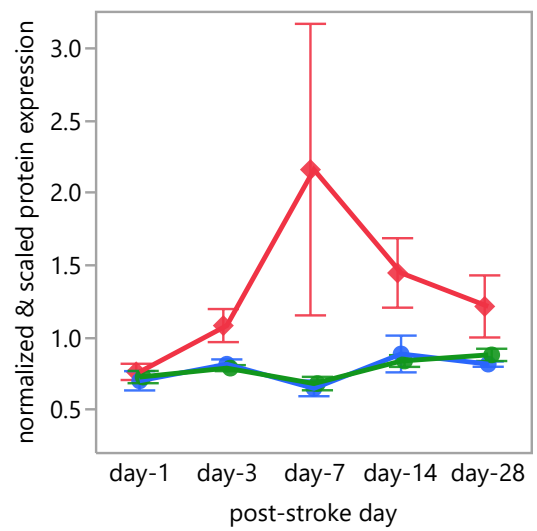

Unique_ID=Ostf1_Q62422

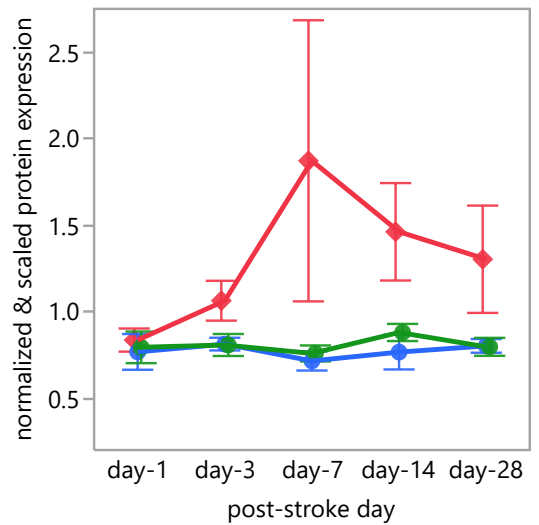

Unique_ID=P01724_P01724

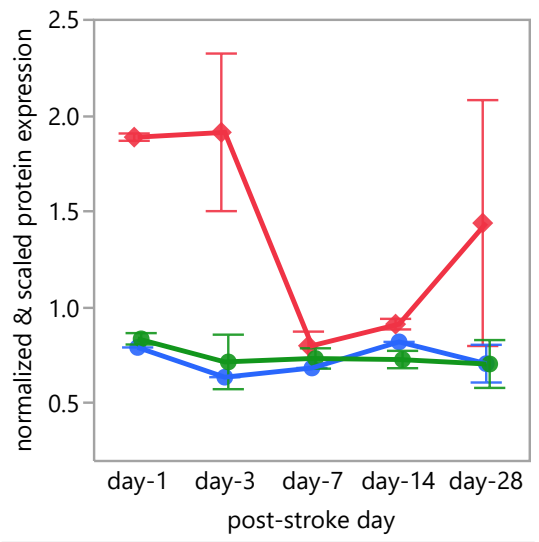

Unique_ID=P03987-2_P03987-2

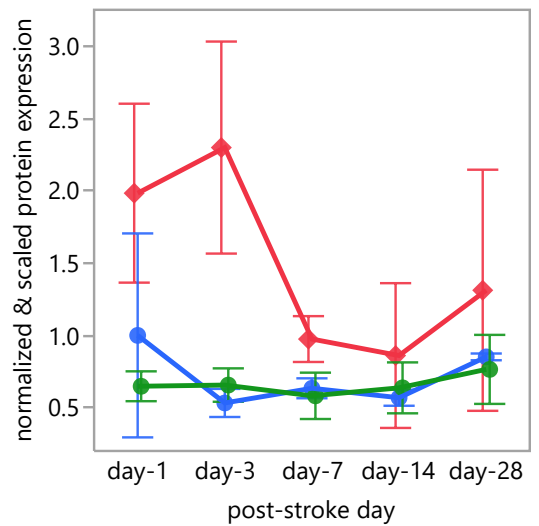




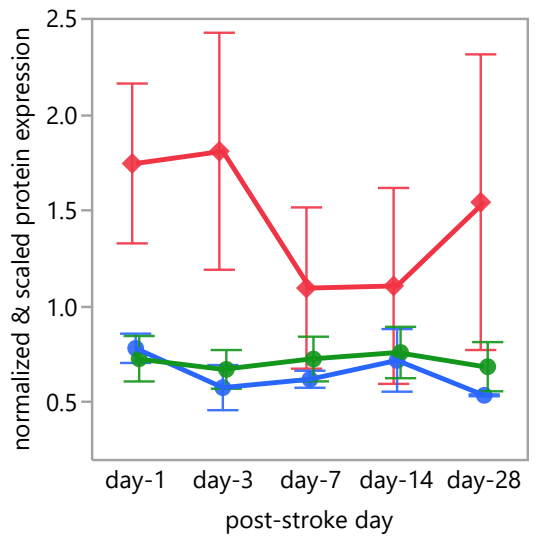

Unique_ID=Parvg_Q9ERD8

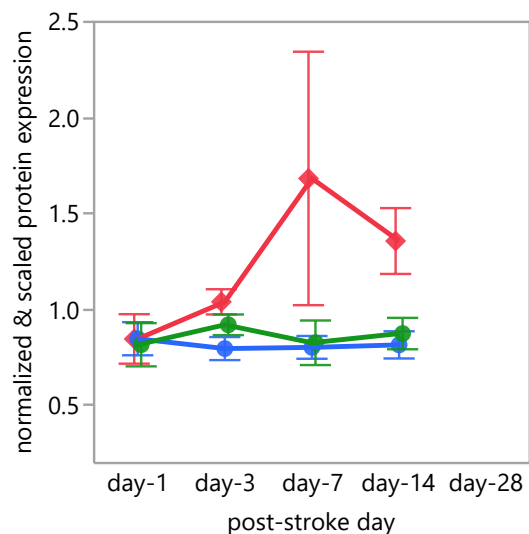

Unique_ID=PIcg2_Q8CIH5

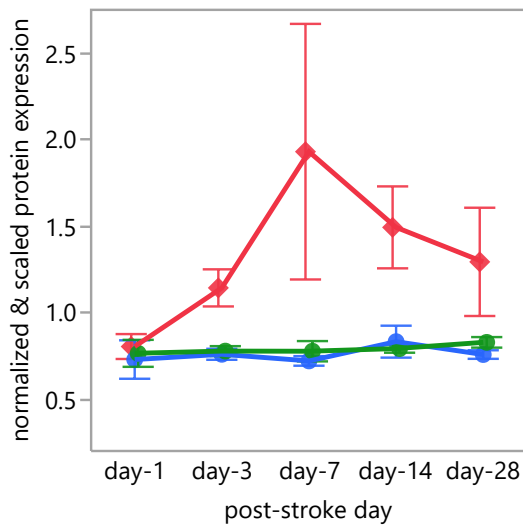

Unique_ID=Plg_P20918

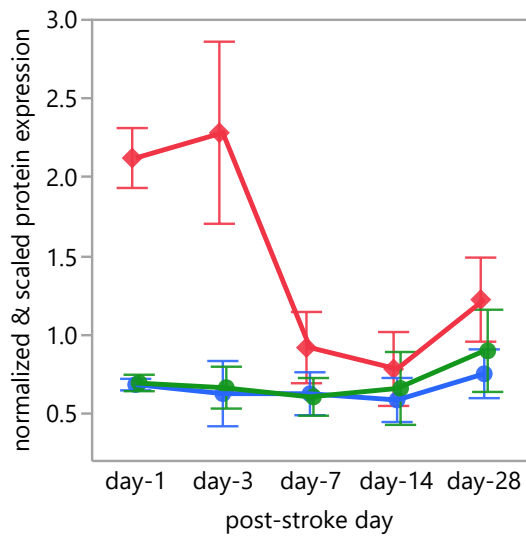

Figure_S2_309TopHits_Profile
Unique_ID=P2rx4_Q9JJX6-4

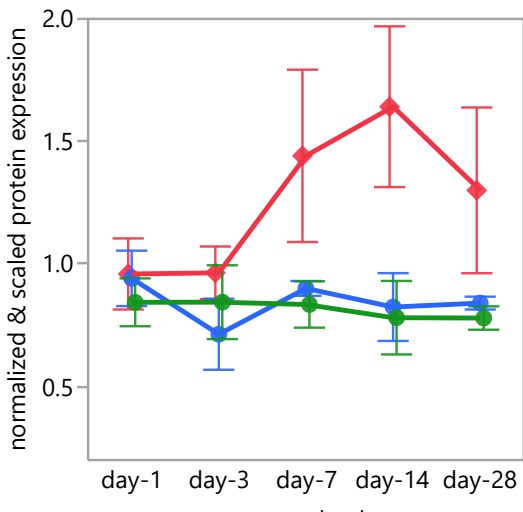

post-stroke day

\section{Unique_ID=Pdia5_Q921X9}

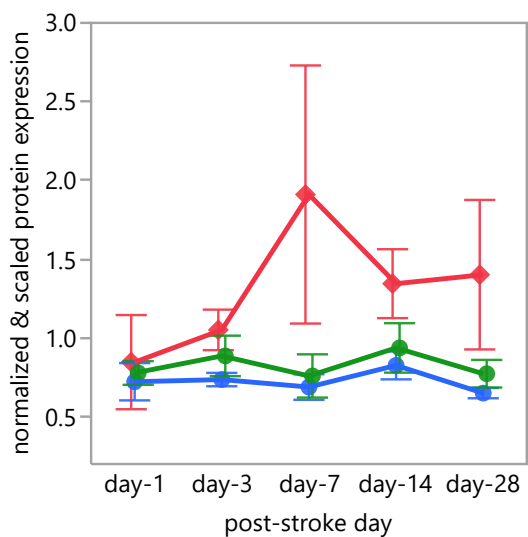

Unique_ID=PId4_Q8BG07-2

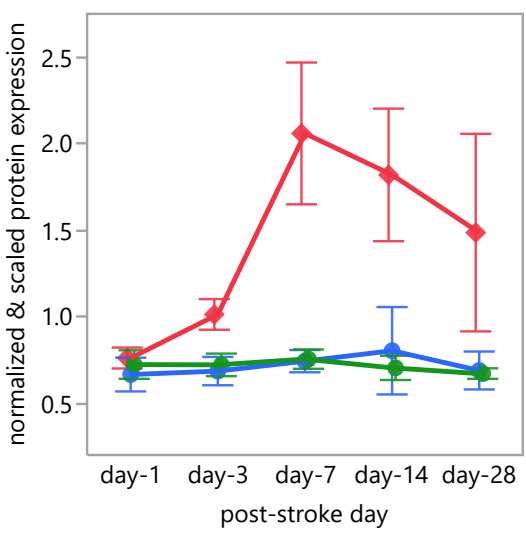

Unique_ID=Plin2_P43883

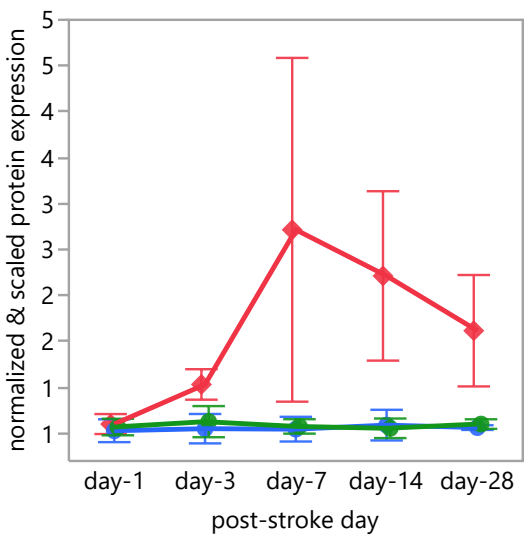

Unique_ID=Palld_Q9ET54-3

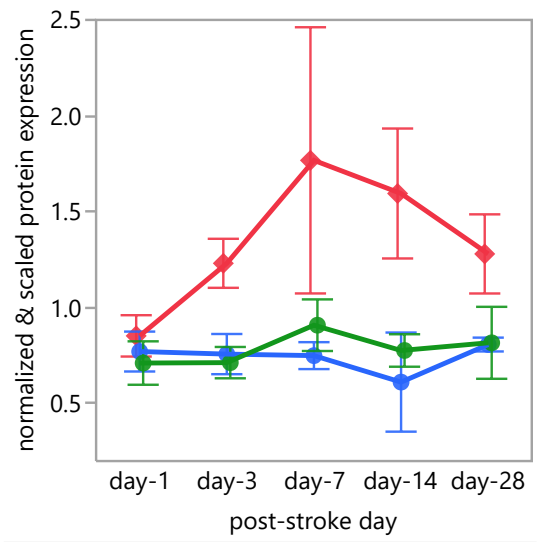

Unique_ID=Pglyrp1_088593

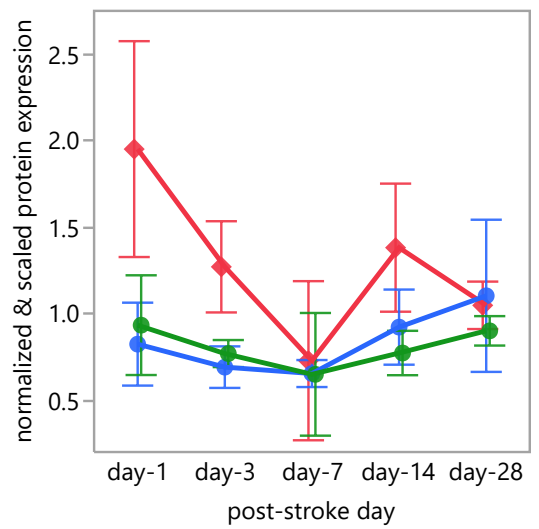

Unique_ID=Plek_Q9JHK5

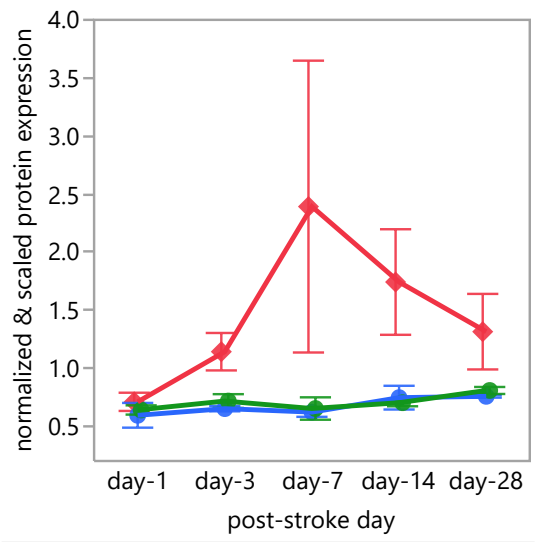

Unique_ID=Plod1_Q9R0E2

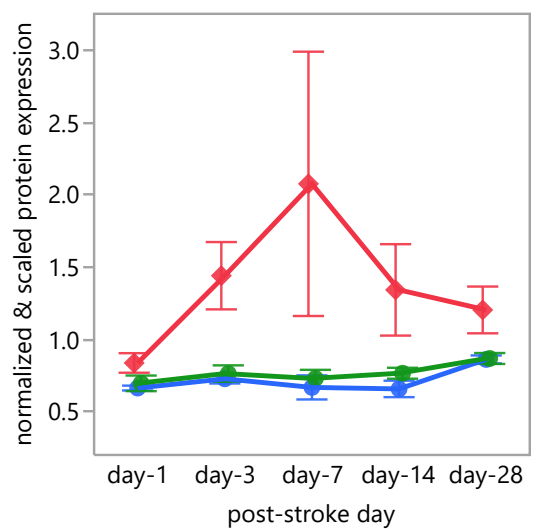


Unique_ID=Pomp_Q9CQT5

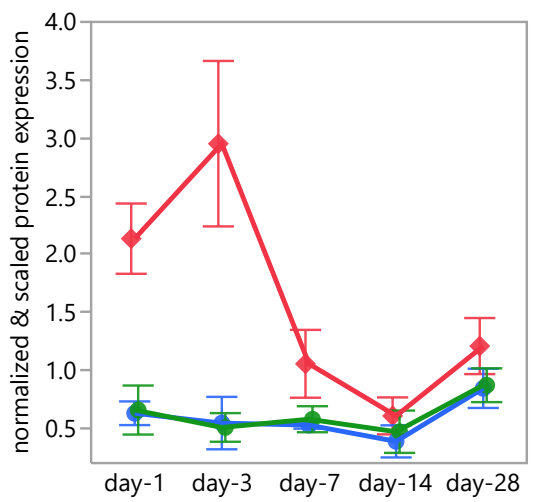

post-stroke day

Unique_ID=Pros1_Q08761

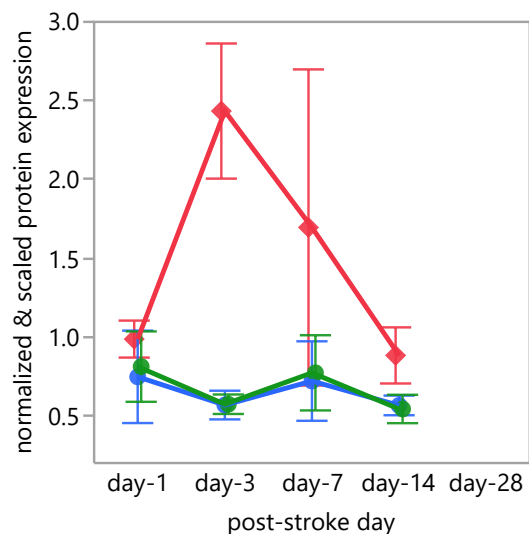

Unique_ID=Psmb10_O35955

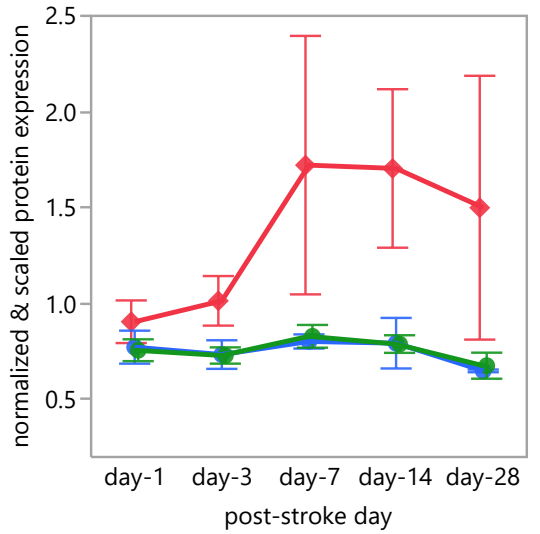

Unique_ID=Ptbp1_P17225

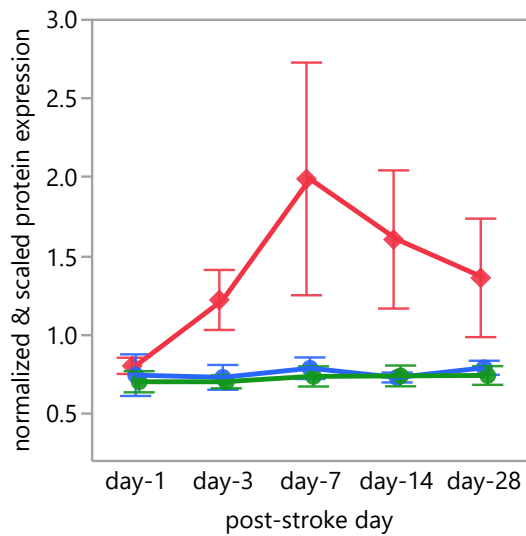

Figure_S2_309TopHits_Profile

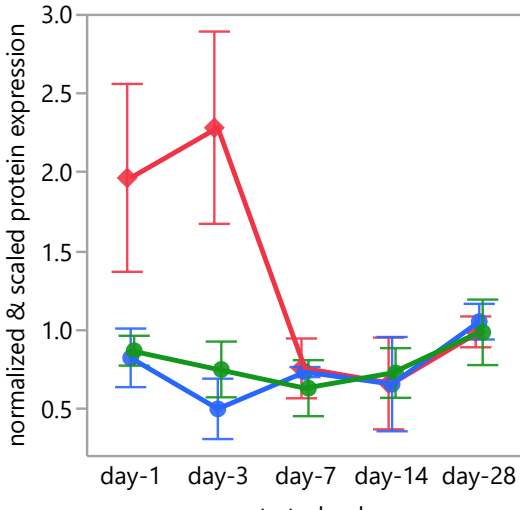

post-stroke day

Unique_ID=Prrt2_E9PUL5

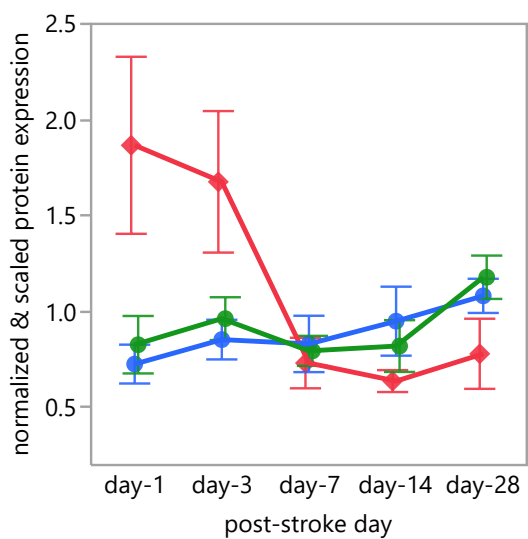

Unique_ID=Psmb8_P28063

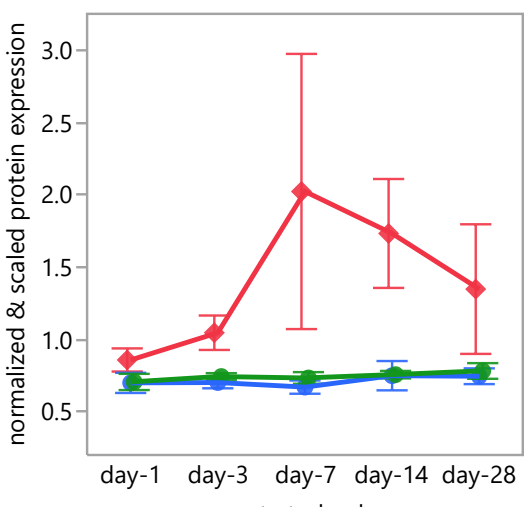

post-stroke day

\section{Unique_ID=Ptgr1_Q91YR9}

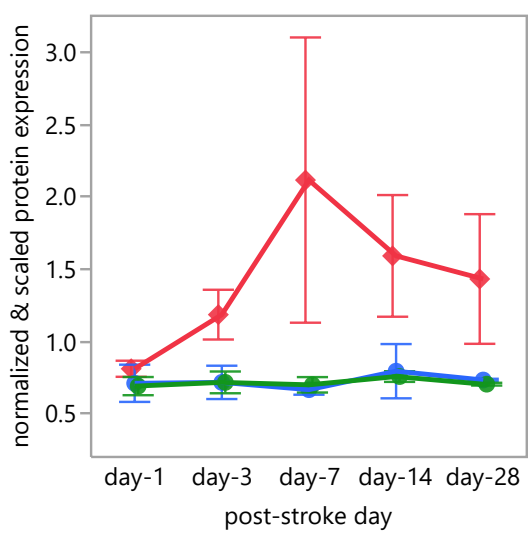

Unique_ID=Ppic_P30412

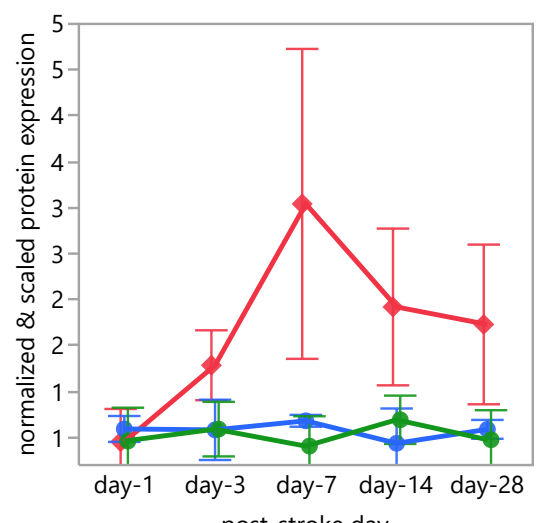

post-stroke day

Unique_ID=Psap_Q61207

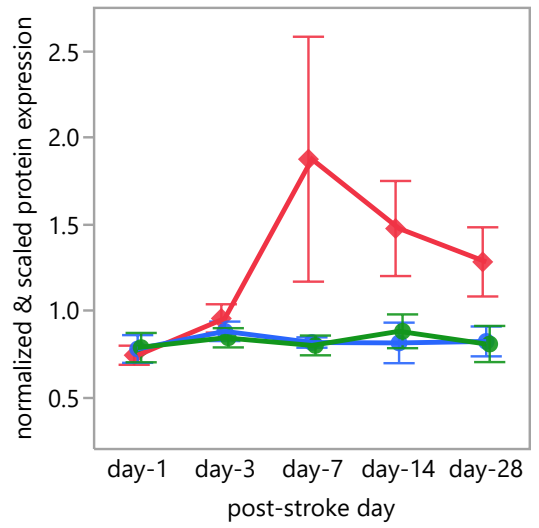

Unique_ID=Psmb9_P28076

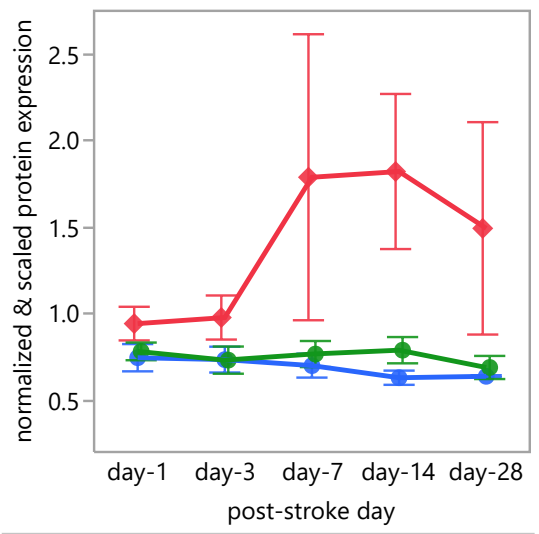

Unique_ID=Ptpn6_P29351

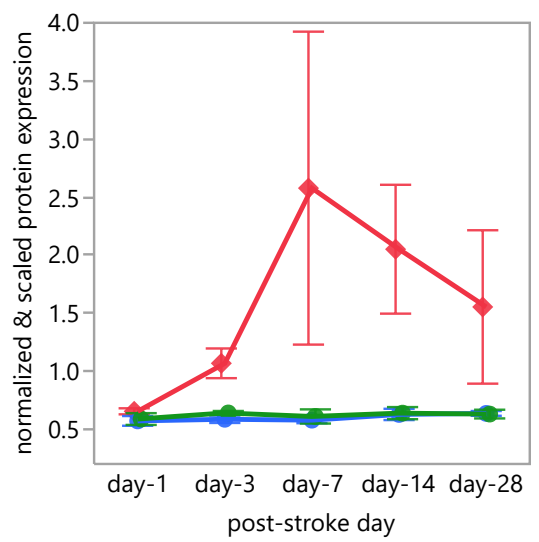




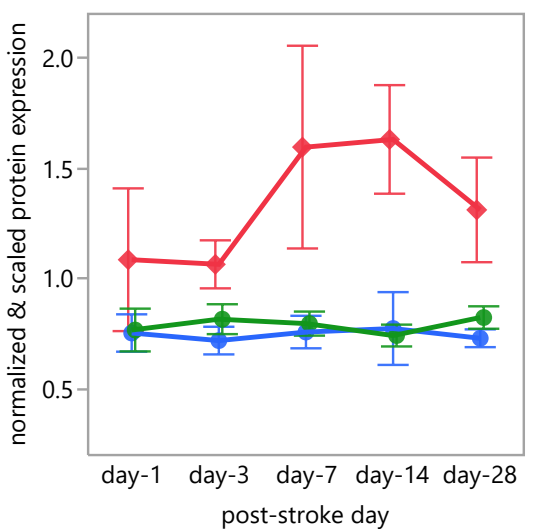

Unique_ID=Rbp4_Q00724

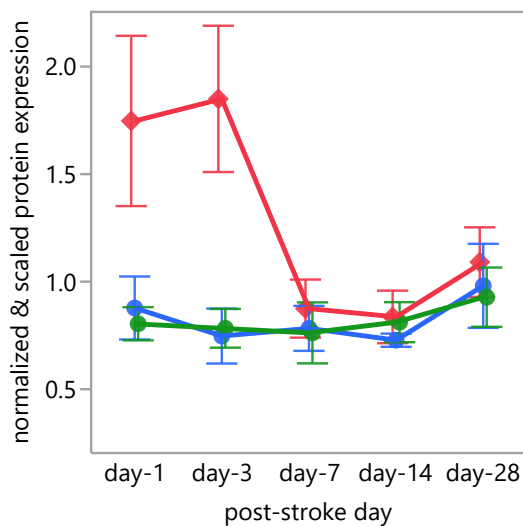

Unique_ID=Rftn1_Q6A0D4

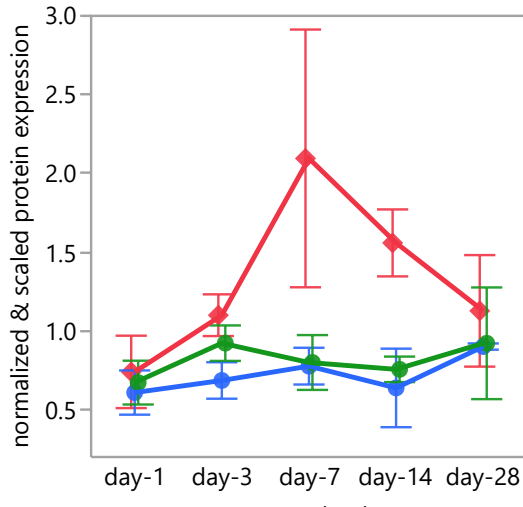

post-stroke day

\section{Unique_ID=Rnaset2_Q9CQ01}

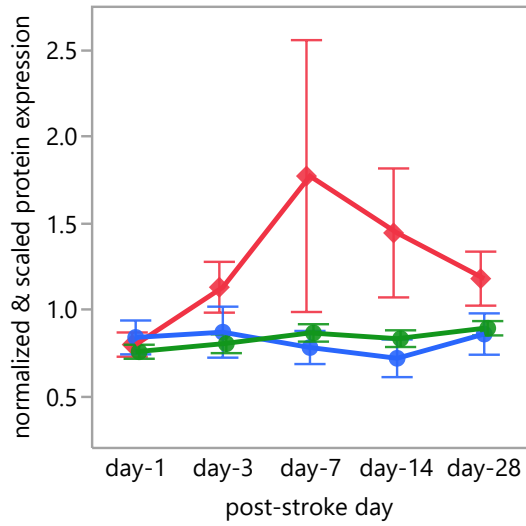

Figure_S2_309TopHits_Profile

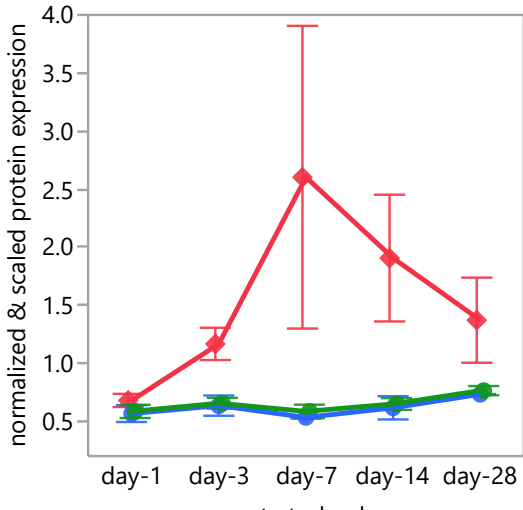

post-stroke day

\section{Unique_ID=Rcn3_Q8BH97}

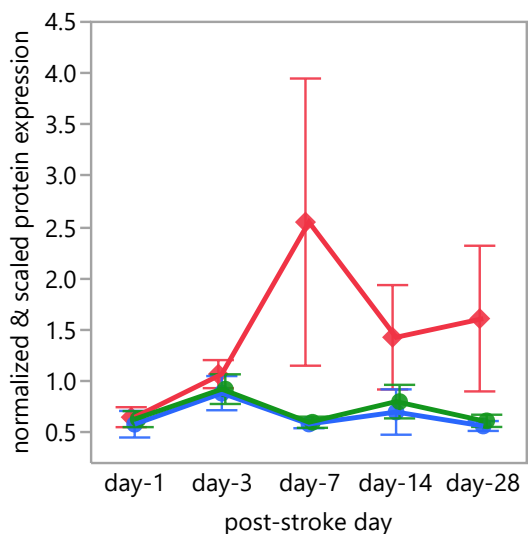

Unique_ID=Ripk1_Q60855

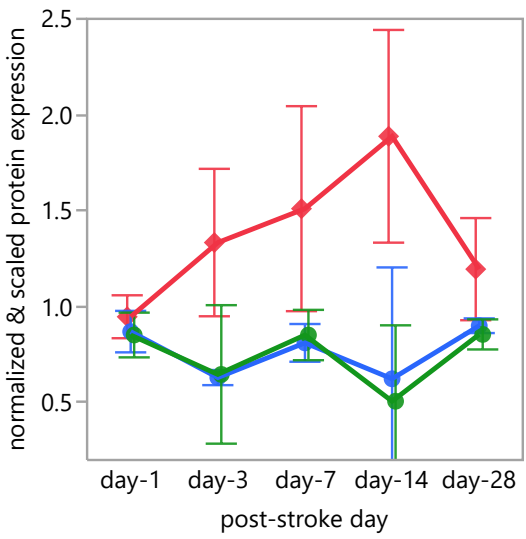

Unique_ID=Rnf213_E9Q555

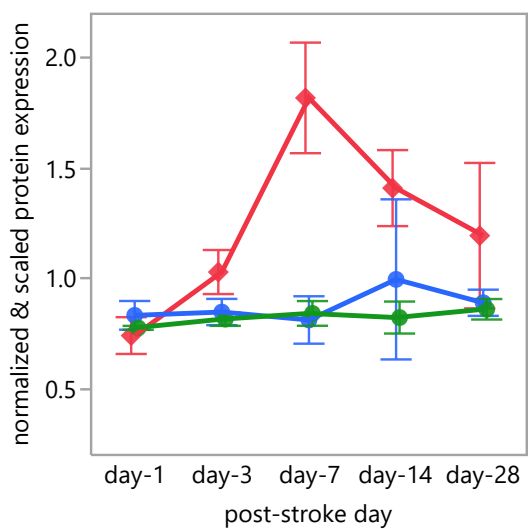

Unique_ID=Rac2_Q05144

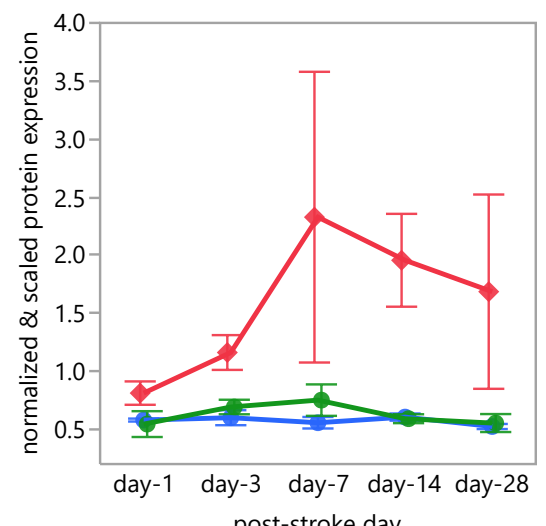

post-stroke day

Unique_ID=Renbp_P82343

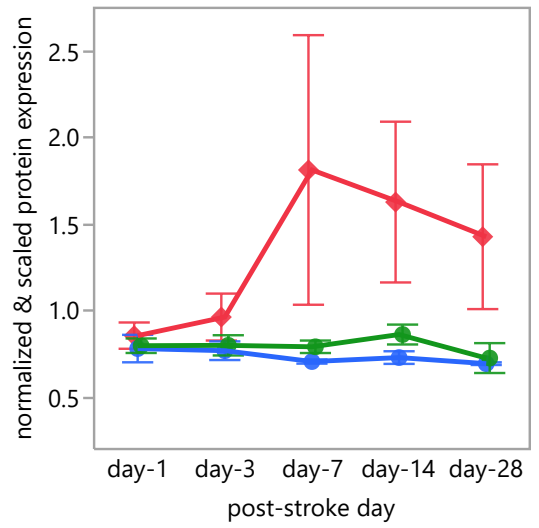

Unique_ID=Rnase4_Q9JJH1

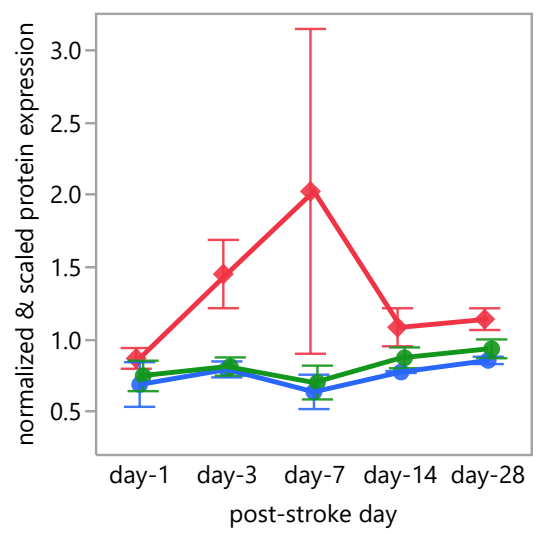

Unique_ID=Rrbp1_Q99PL5

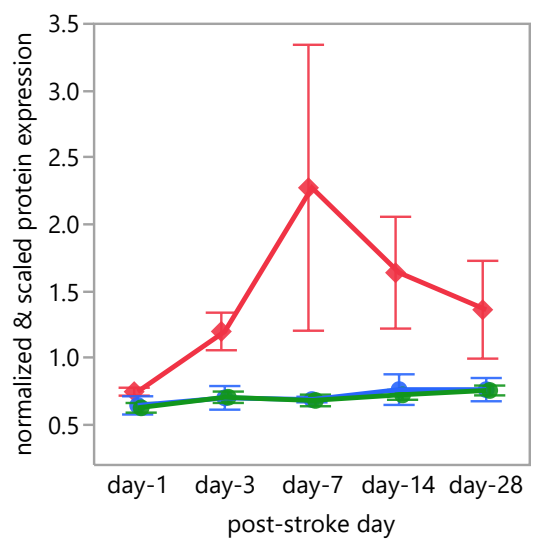


Unique_ID=S100a11_P50543

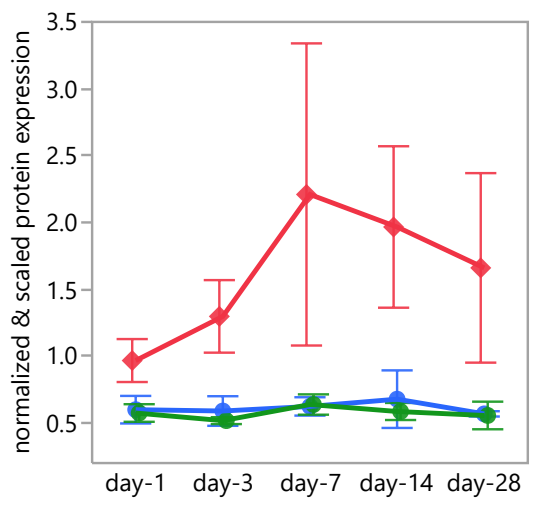

post-stroke day

\section{Unique_ID=S100a8_P27005}

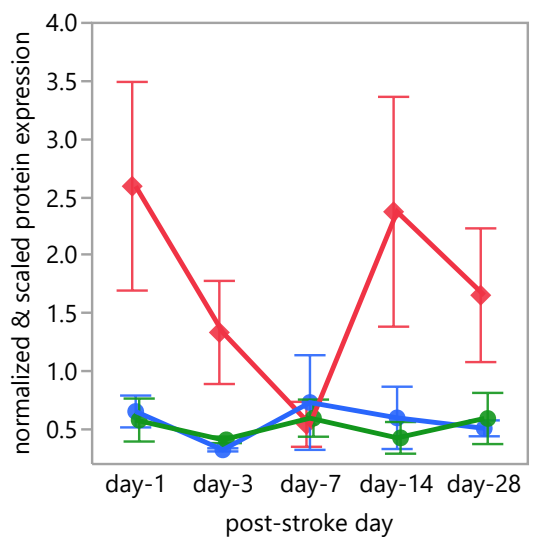

Unique_ID=Scpep1_Q920A5

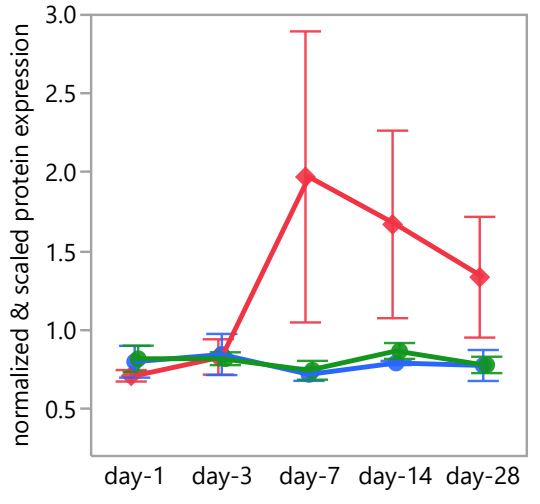

post-stroke day

\section{Unique_ID=Serpina1a_P07758}

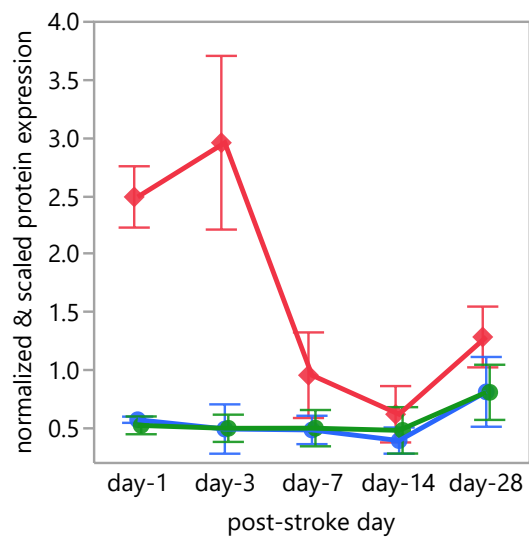

Figure_S2_309TopHits_Profile

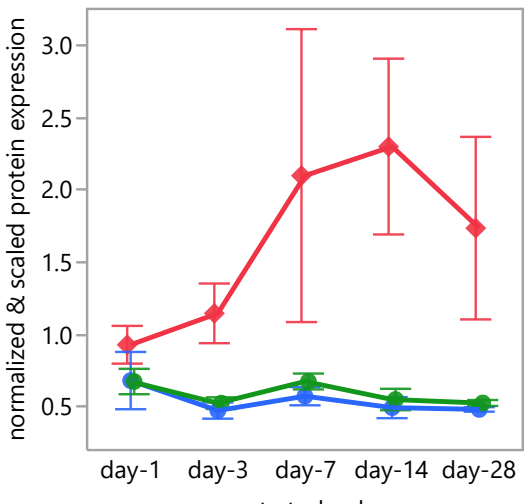

post-stroke day

Unique_ID=S100a9_P31725

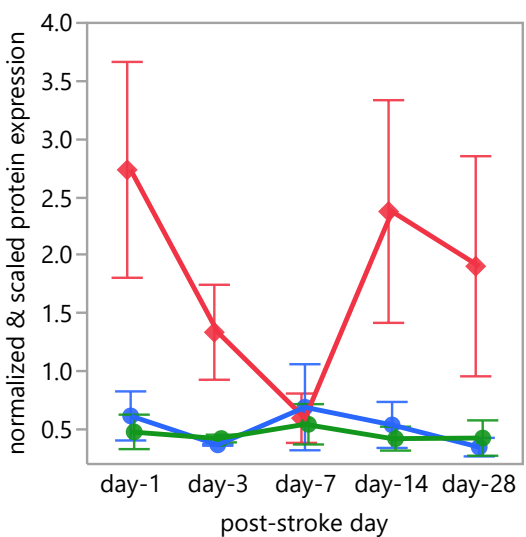

Unique_ID=Sepp1_P70274

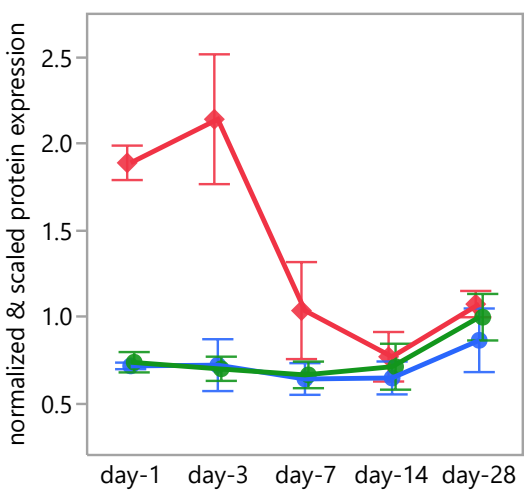

post-stroke day

\section{Unique_ID=Serpina1b_P22599}

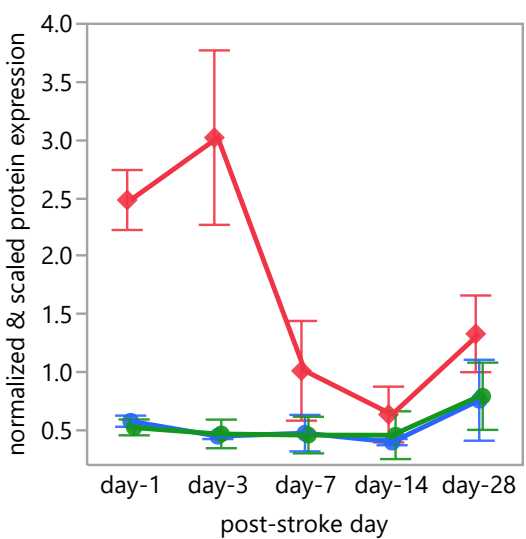

Unique_ID=S100a6_P14069

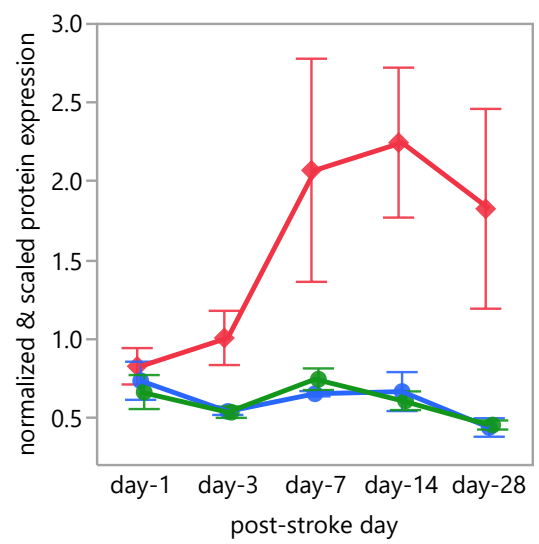

Unique_ID=Sash3_Q8K352

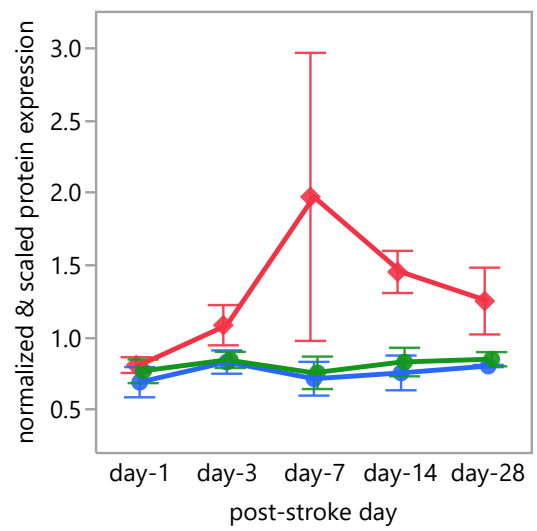

Unique_ID=Serpina10_Q8R121

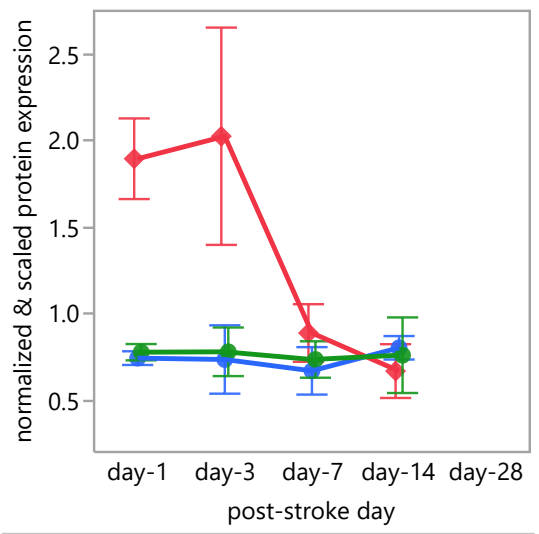

Unique_ID=Serpina1d_Q00897

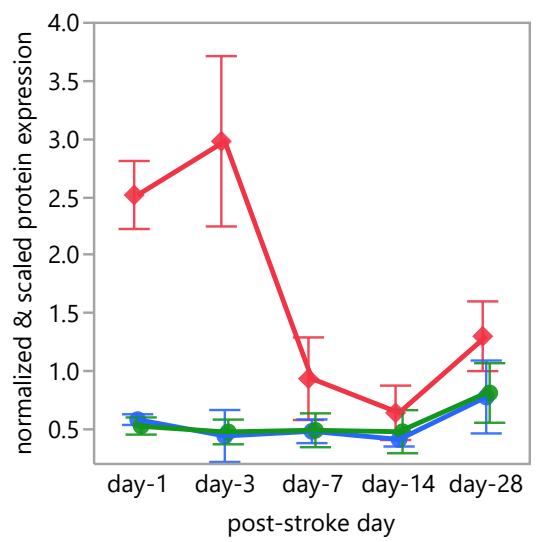




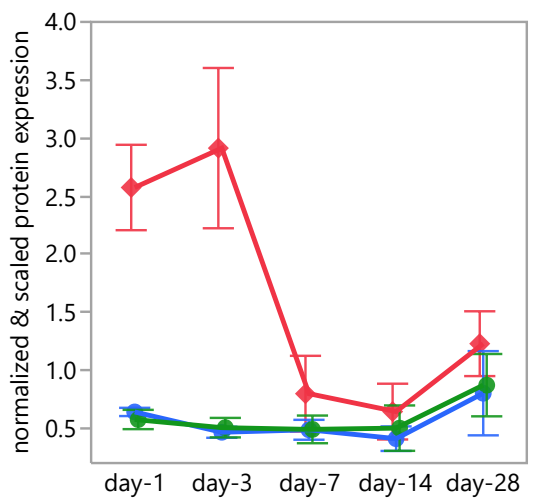

post-stroke day

Unique_ID=Serpina3n_Q91WP6

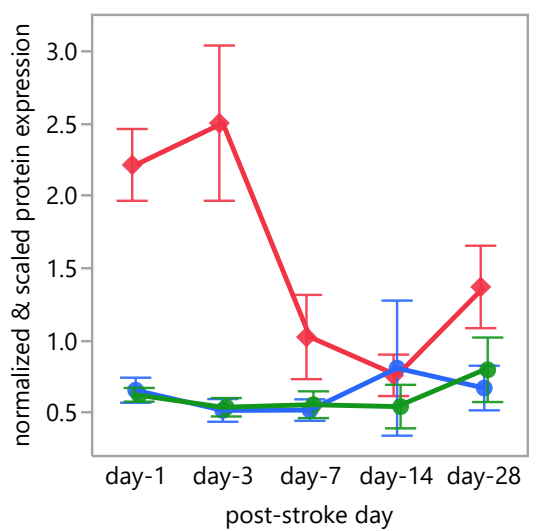

Unique_ID=Serpind1_P49182

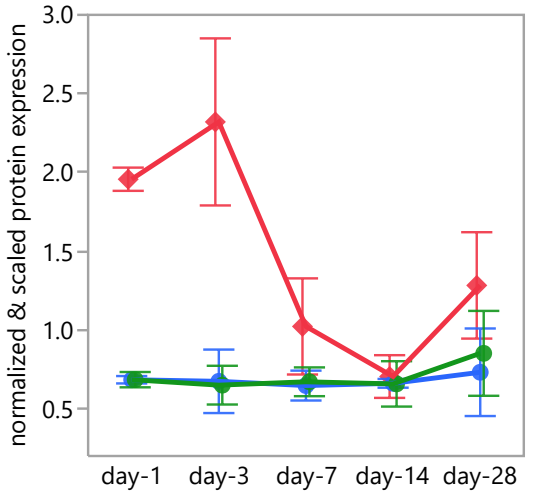

post-stroke day

Unique_ID=Serping1_P97290

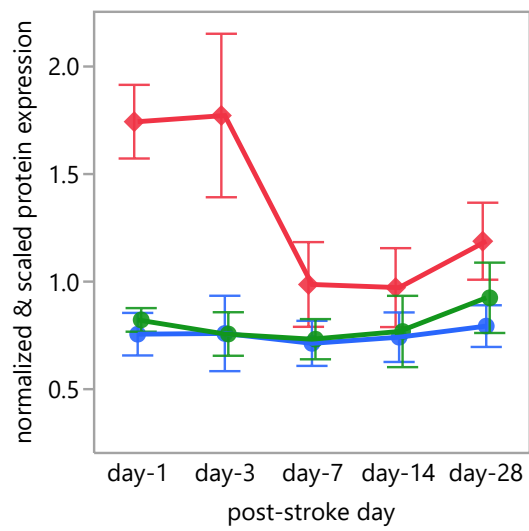

Figure_S2_309TopHits_Profile

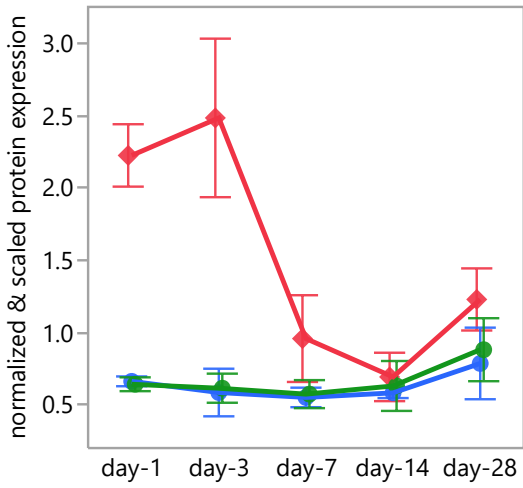

post-stroke day

Unique_ID=Serpina6_Q06770

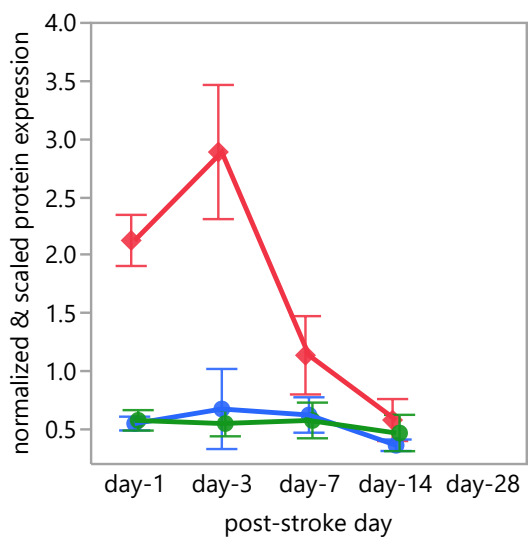

Unique_ID=Serpinf1_P97298

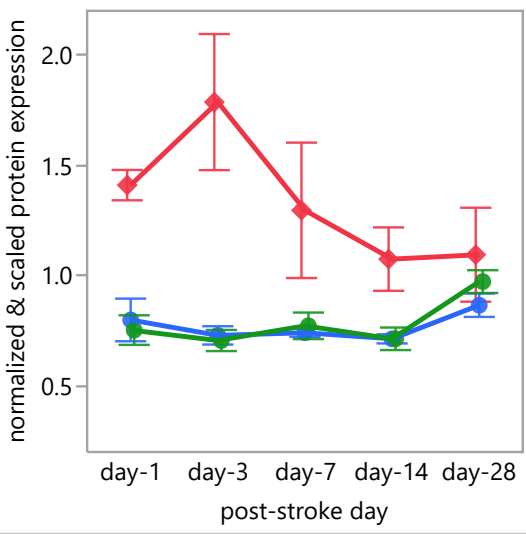

Unique_ID=Serpinh1_P19324

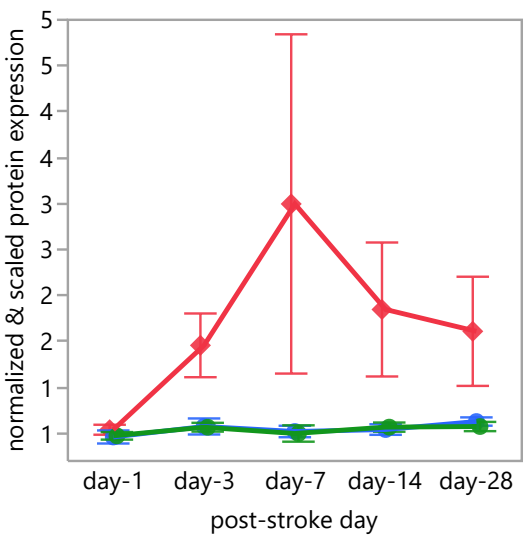

Unique_ID=Serpina3m_Q03734

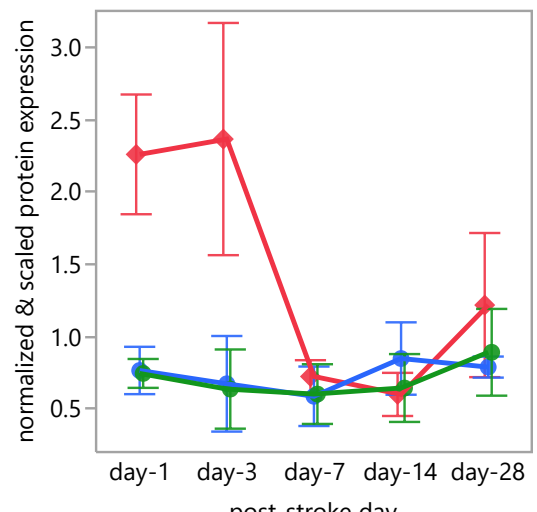

post-stroke day

Unique_ID=Serpinc1_P32261

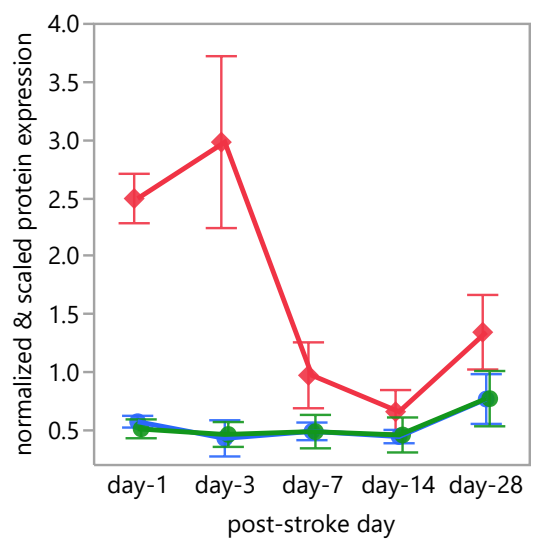

Unique_ID=Serpinf2_Q61247

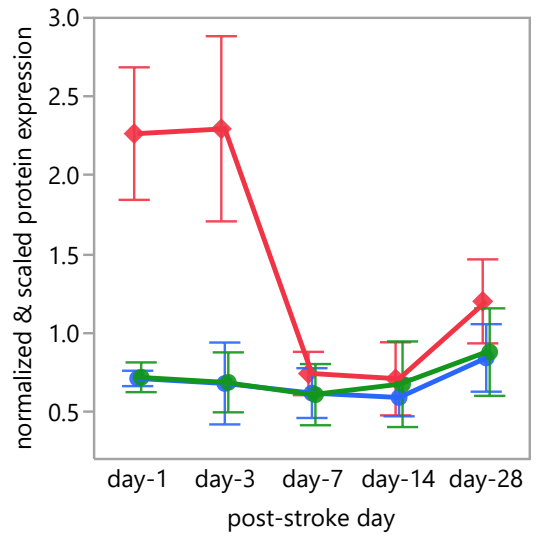

Unique_ID=Sgpl1_Q8R0X7

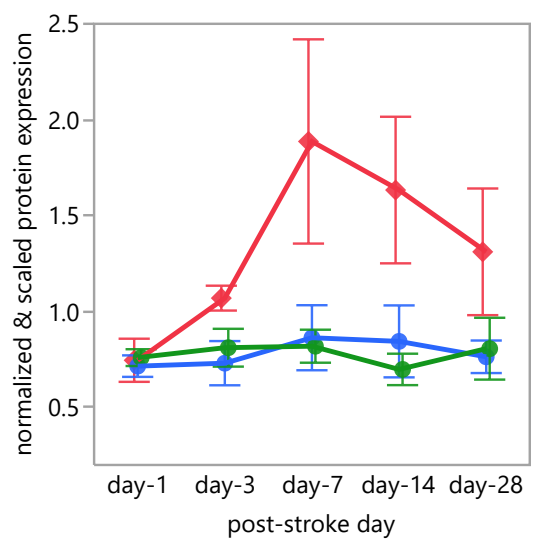


Unique_ID=Siglec1_Q62230-3

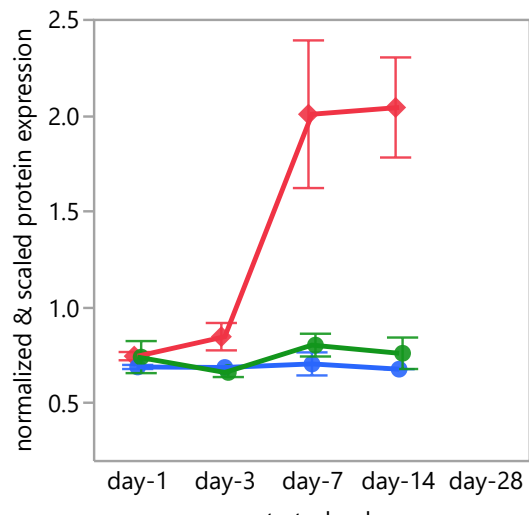

post-stroke day

Unique_ID=Stab1_Q8R4Y4-2

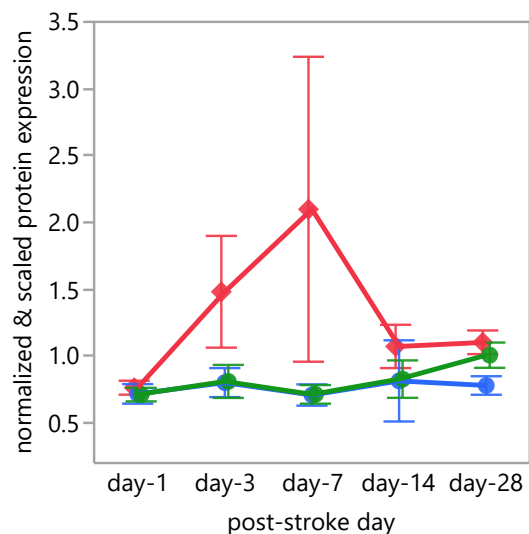

Unique_ID=Stxbp2_Q64324

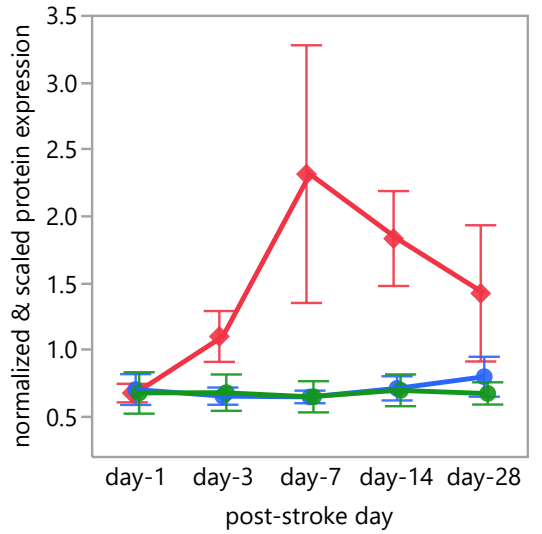

Unique_ID=Tapbp_Q9R233-2

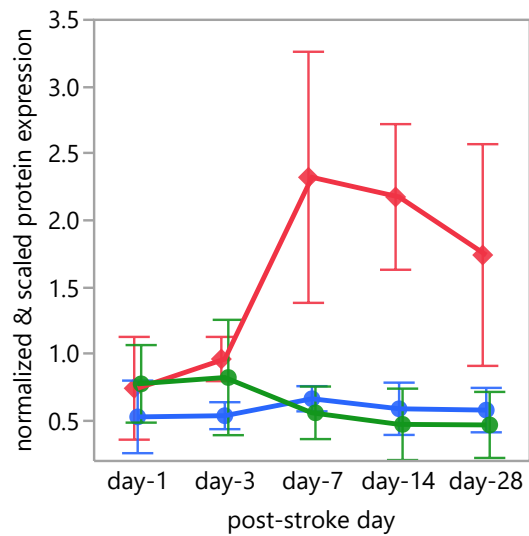

Figure_S2_309TopHits_Profile

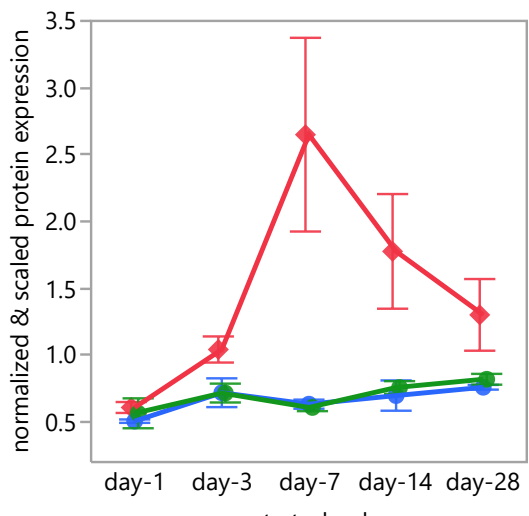

post-stroke day

\section{Unique_ID=Stat1_P42225}

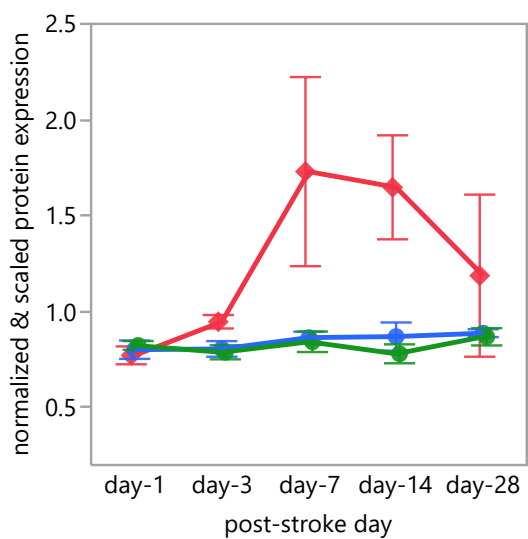

Unique_ID=Syk_P48025

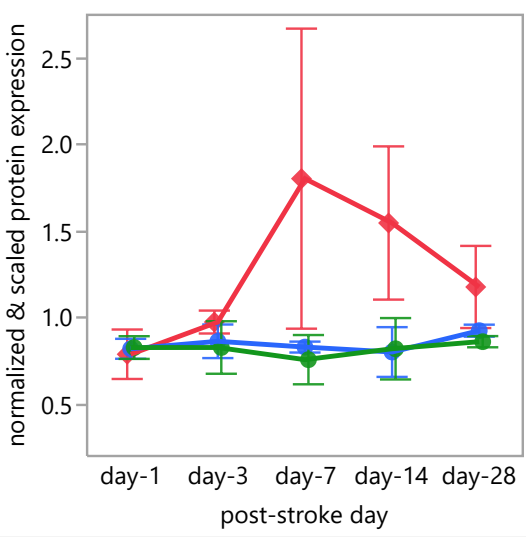

Unique_ID=Tcn2_088968

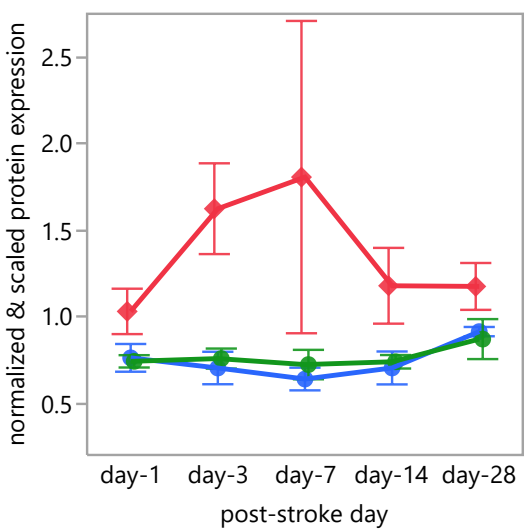

Unique_ID=Spp1_P10923

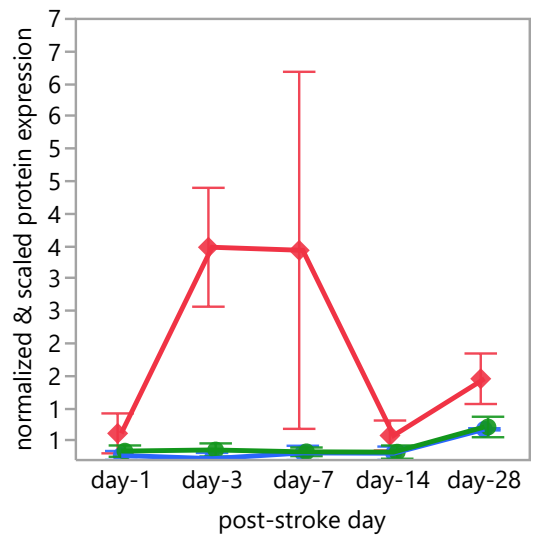

Unique_ID=Steap3_Q8CI59

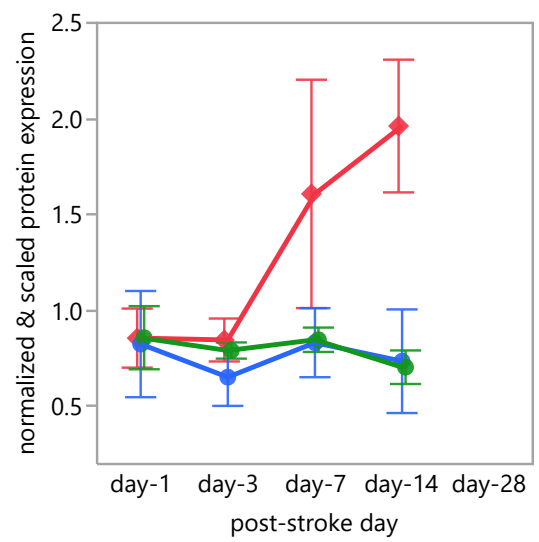

Unique_ID=Tagln2_Q9WVA4

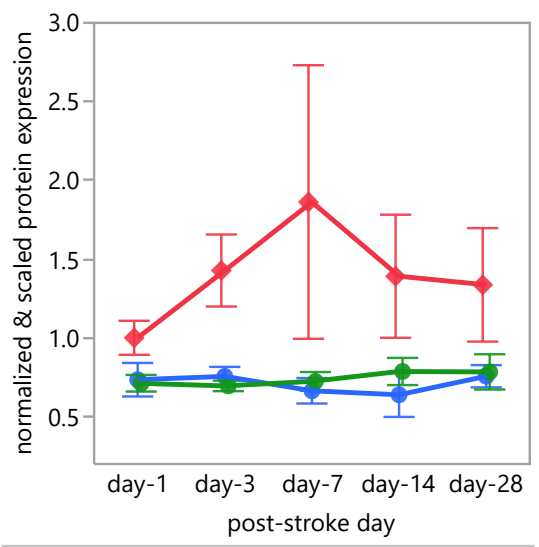

Unique_ID=Tes_P47226-2

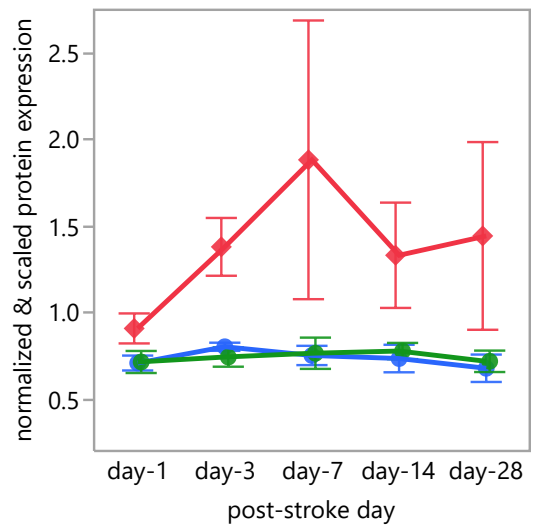


Unique_ID=Tf_Q921I1

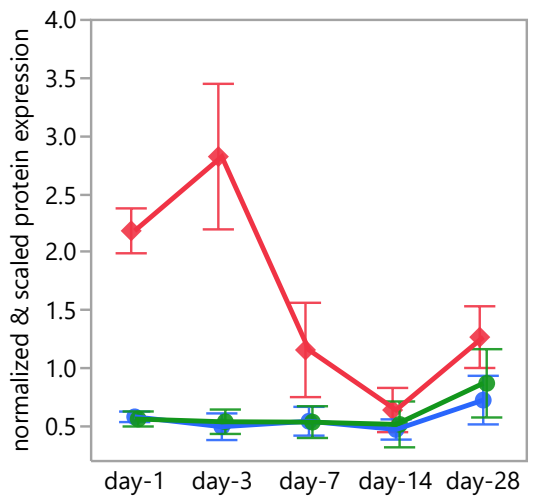

post-stroke day

\section{Unique_ID=Thbs1_P35441}

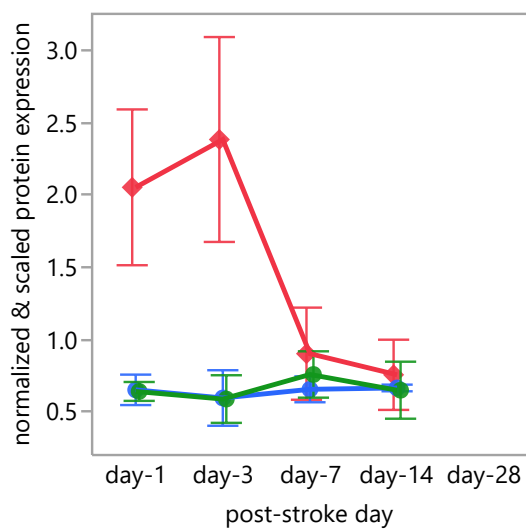

Unique_ID=Tpm4_Q6IRU2

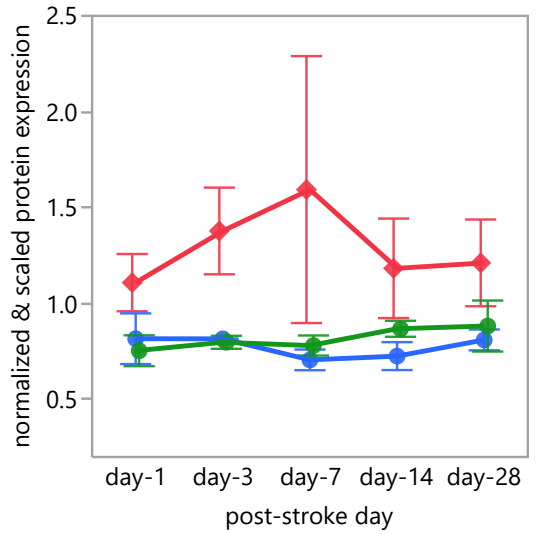

Unique_ID=Uap1I1_Q3TW96

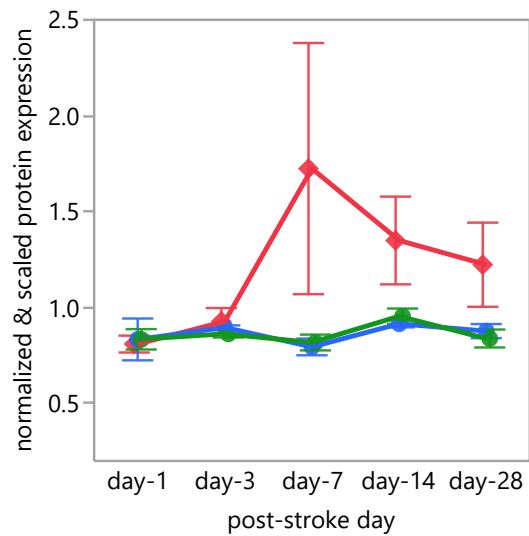

Figure_S2_309TopHits_Profile
Unique_ID=Tgfbi_P82198

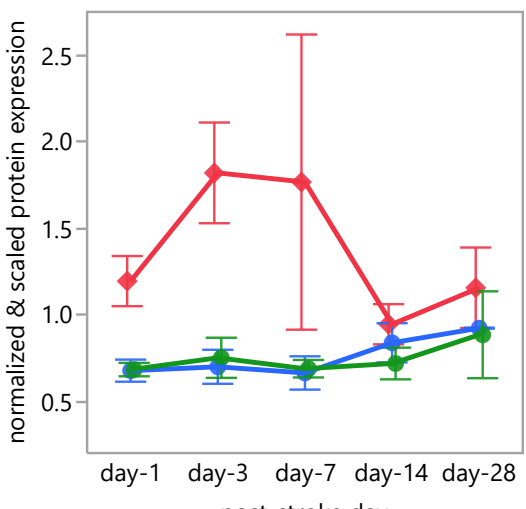

Unique_ID=TIn1_P26039

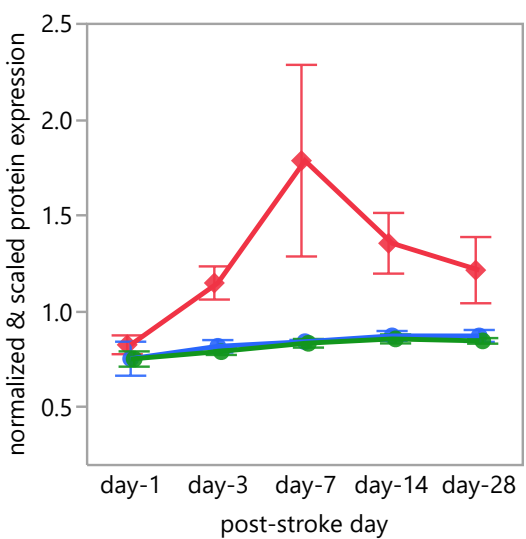

Unique_ID=Trem2_Q99NH8

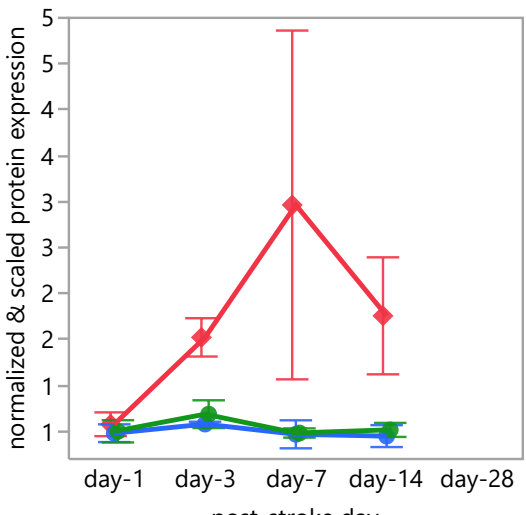

post-stroke day

Unique_ID=Ugt1a7c_Q6ZQM8

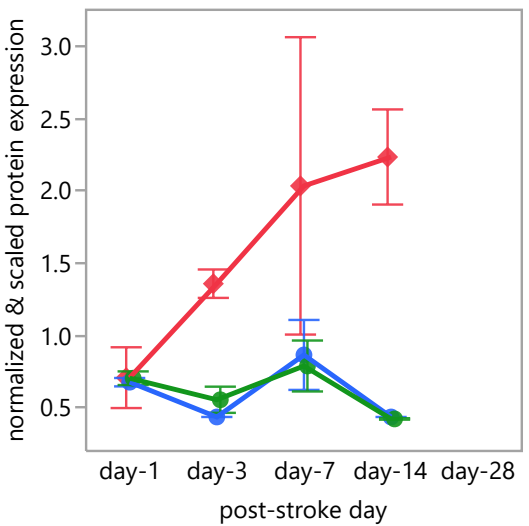

Unique_ID=Tgm1_Q9JLF6

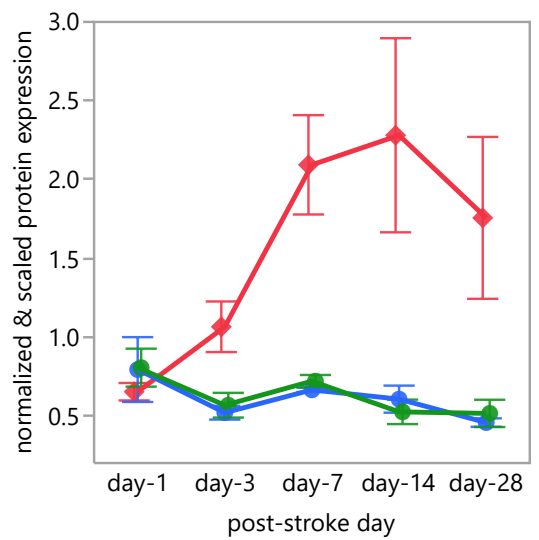

Unique_ID=Tmod3_Q9JHJO

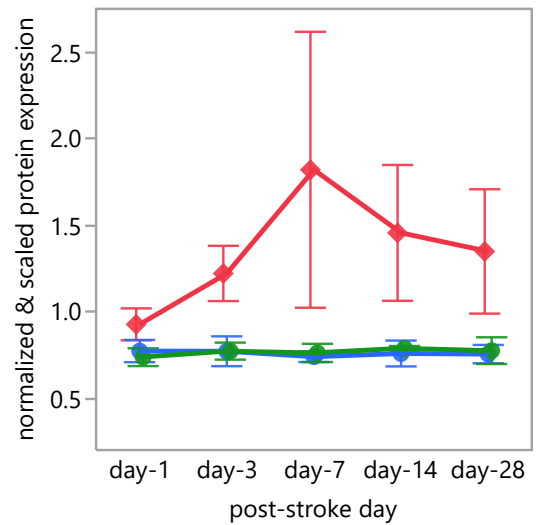

Unique_ID=Ttr_P07309

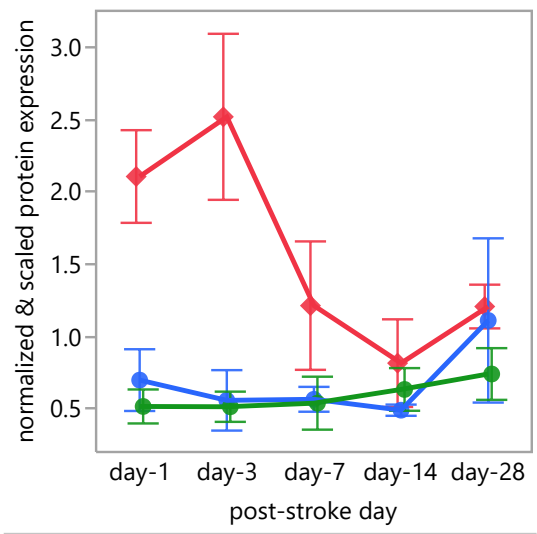

Unique_ID=Vamp8_070404

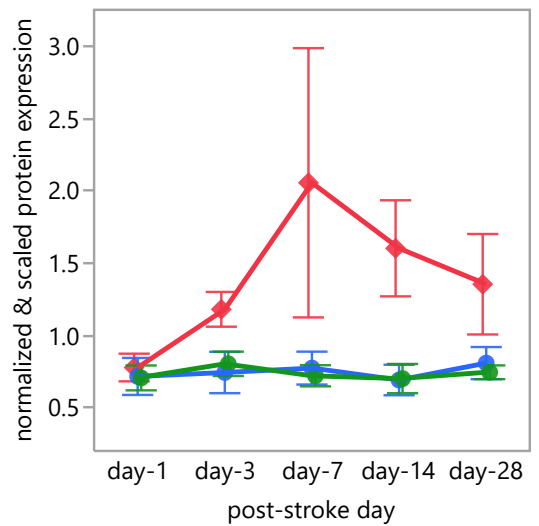




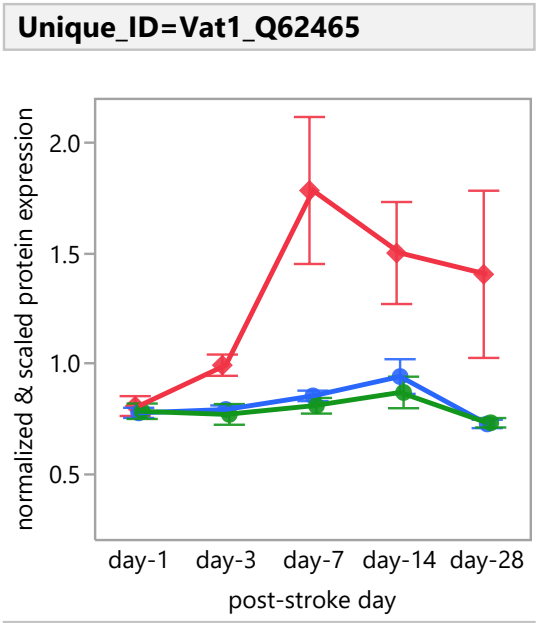

Unique_ID=Vtn_P29788

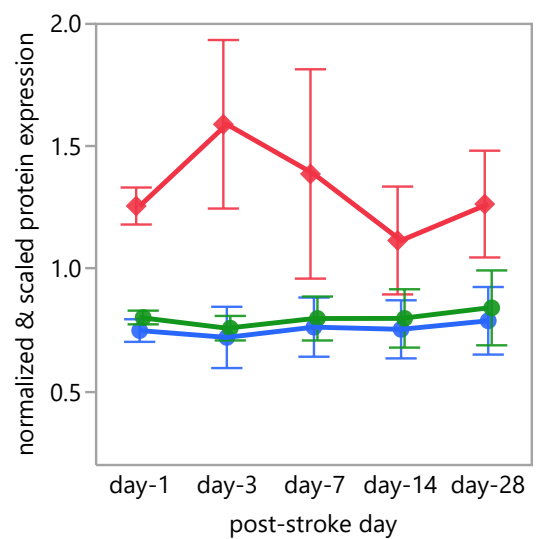

Unique_ID=Ybx1_P62960

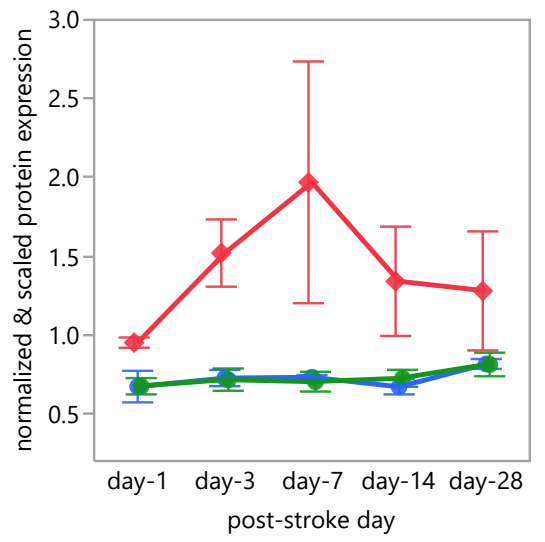

Unique_ID=Vav1_P27870

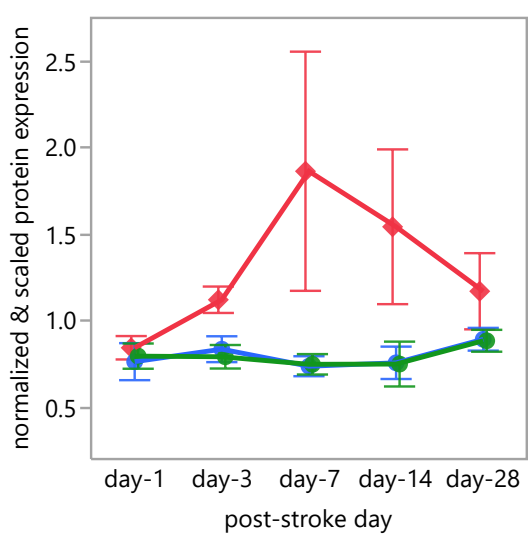

Unique_ID=Was_P70315

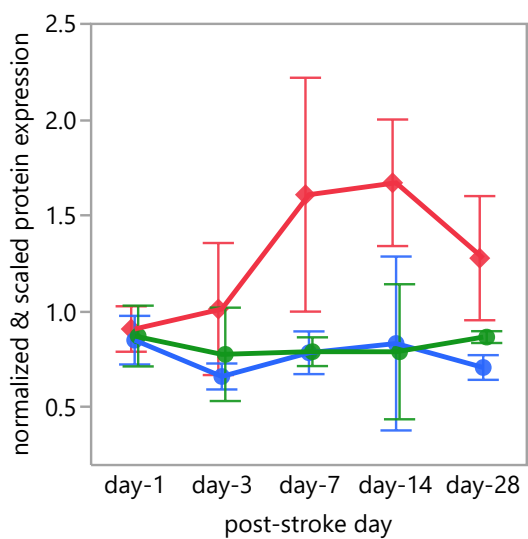

Unique_ID=Zadh2_Q8BGC4

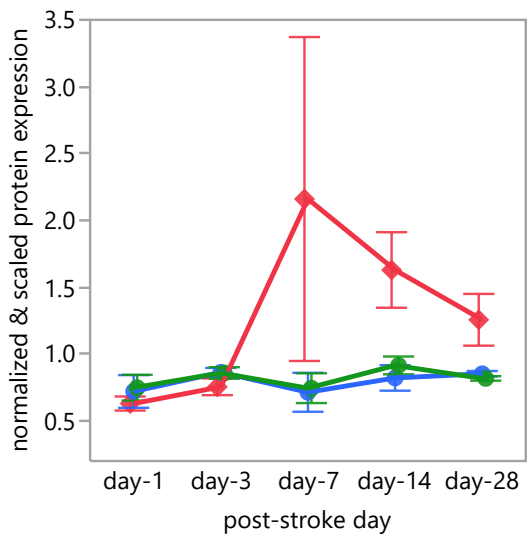

Unique_ID=Vim_P20152

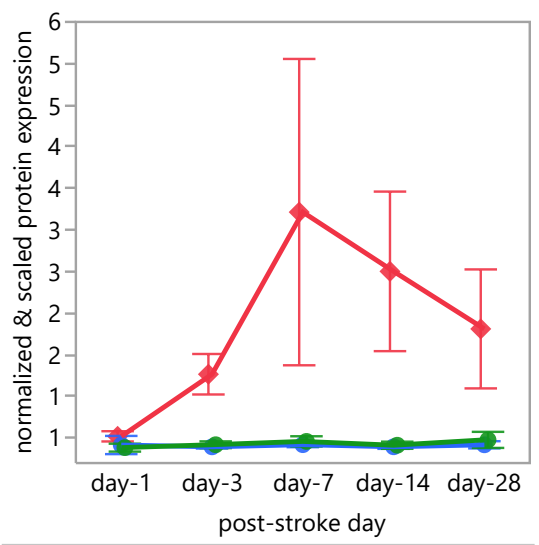

Unique_ID=Wipi1_Q8R3E3-2

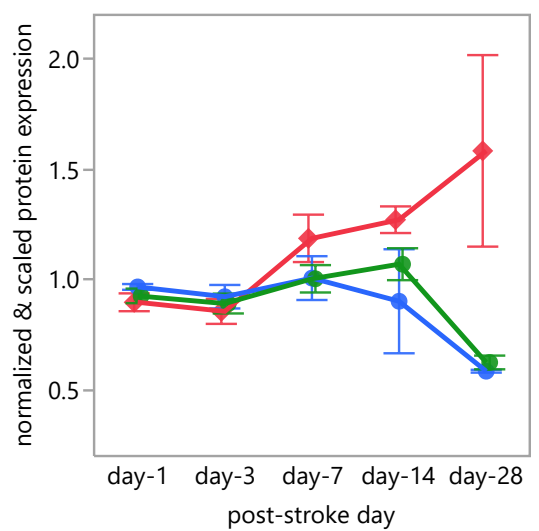

Unique_ID=Zbp1_Q9QY24

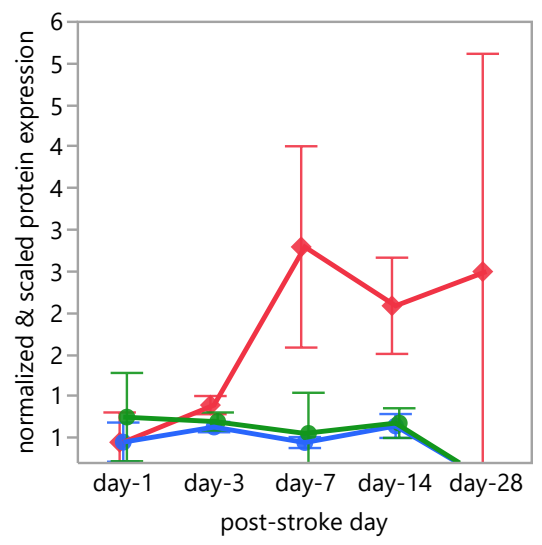


Figure S3. Western Blots of Albumin in the Brain Cortex Tissue Samples.

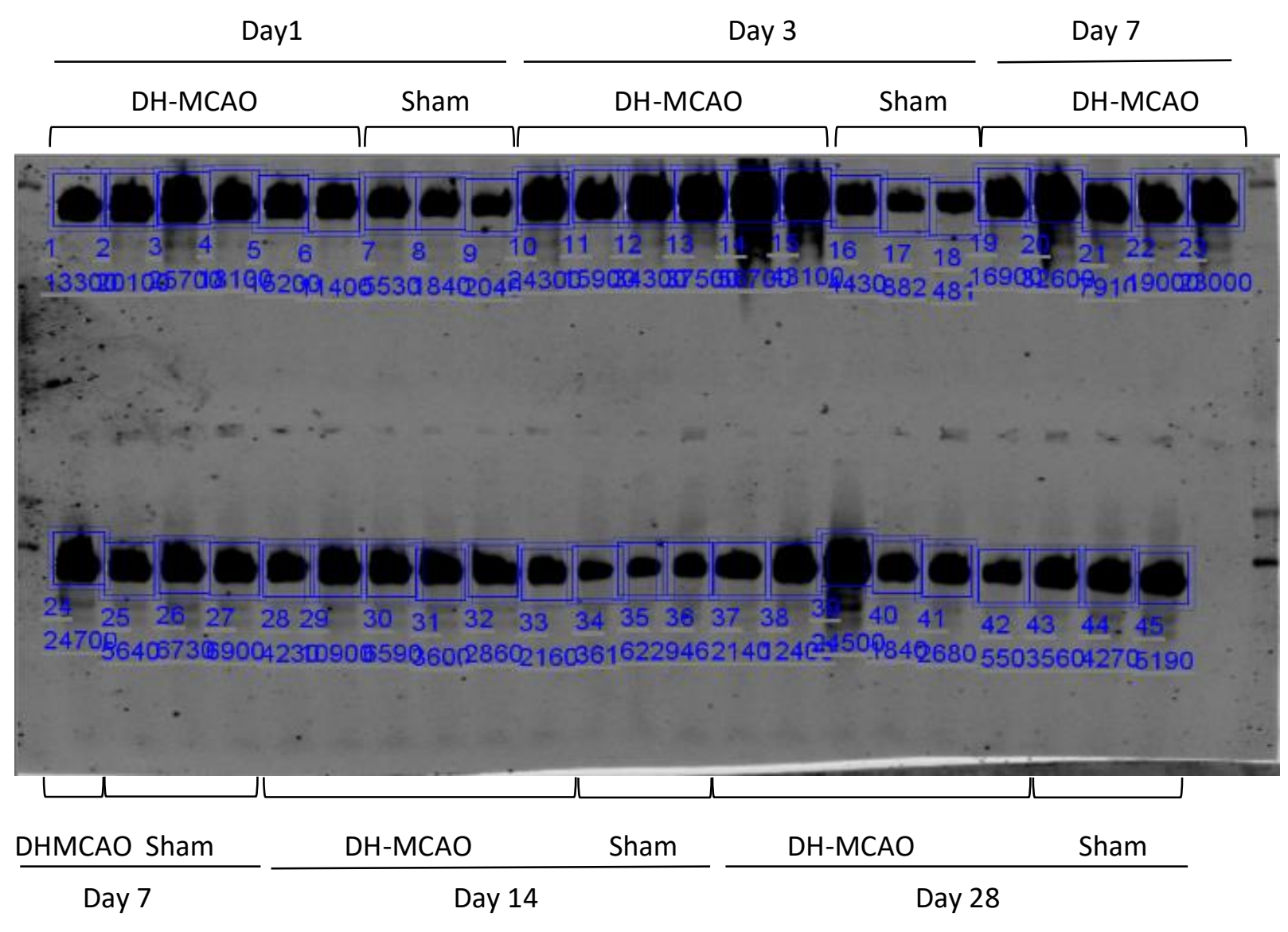

